Prepared in cooperation with U.S. Army Corps of Engineers

\title{
Historical Streamflow and Stage Data Compilation for the Lower Columbia River, Pacific Northwest
}

Open-File Report 2020-1138 

Prepared in cooperation with U.S. Army Corps of Engineers

\section{Historical Streamflow and Stage Data Compilation for the Lower Columbia River, Pacific Northwest}

By Carrie L. Boudreau, Marc A. Stewart, and Adam J. Stonewall

Open-File Report 2020-1138

U.S. Department of the Interior

U.S. Geological Survey 
U.S. Geological Survey, Reston, Virginia: 2021

For more information on the USGS-the Federal source for science about the Earth, its natural and living resources, natural hazards, and the environment-visit https://www.usgs.gov/ or call 1-888-ASK-USGS (1-888-275-8747).

For an overview of USGS information products, including maps, imagery, and publications, visit https://store.usgs.gov/.

Any use of trade, firm, or product names is for descriptive purposes only and does not imply endorsement by the U.S. Government.

Although this information product, for the most part, is in the public domain, it also may contain copyrighted materials as noted in the text. Permission to reproduce copyrighted items must be secured from the copyright owner.

Suggested citation:

Boudreau, C.L., Stewart, M.A., and Stonewall, A.J., 2021, Historical streamflow and stage data compilation for the Lower Columbia River, Pacific Northwest: U.S. Geological Survey Open-File Report 2020-1138, 50 p., https://doi.org/10.3133/ofr20201138.

Data release:

Boudreau, C.L., Stewart, M.A., and Stonewall, A.J., 2021, Historical streamflow and stage data for the Lower Columbia River Basin and the coasts of Washington, Oregon, and Northern California: U.S. Geological Survey data release, https://doi.org/10.5066/P9R6RT0Z. 


\section{Acknowledgments}

The authors thank the following U.S. Geological Survey staff for their assistance in compiling the datasets included in this report: Lisa Hoaks, Gabriel Gordon, Heather Bervid, and Lindsey Arotin. They also thank Patrick Haluska (also U.S. Geological Survey) for creating the maps in this report and Ryan Cahill (U.S. Army Corps of Engineers) for supplying some historical data from the U.S. Army Corps of Engineers archives. 


\section{Contents}

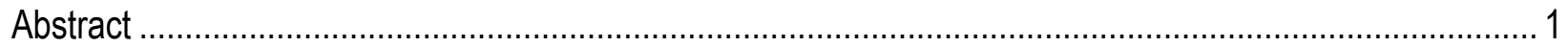

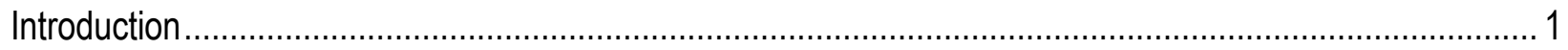

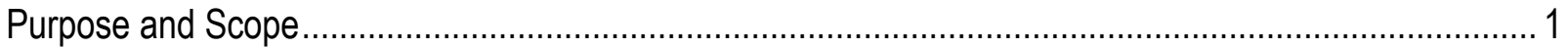

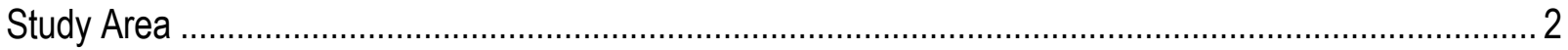

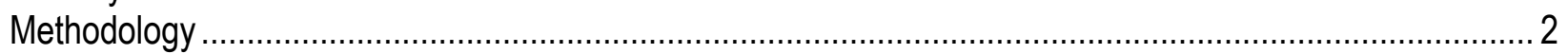

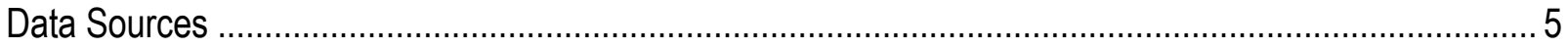

United States Geological Survey (USGS) ……………….............................................. 5

Oregon Water Resources Department (OWRD) .................................................................. 10

National Oceanic and Atmospheric Administration (NOAA) ......................................................... 14

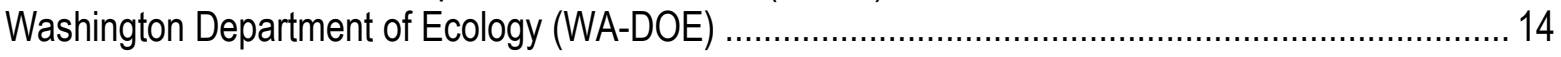

Pacific Northwest National Laboratory (PNNL) ………….................................................... 14

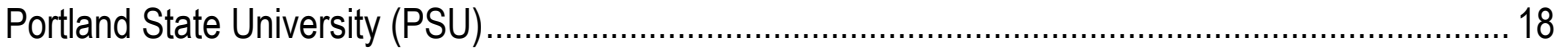

United States Army Corps of Engineers (USACE) Dataquery ..................................................... 18

Review and Quality Assurance/Quality Control (QA/QC) of Acquired Datasets .................................... 20

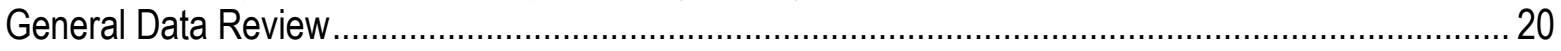

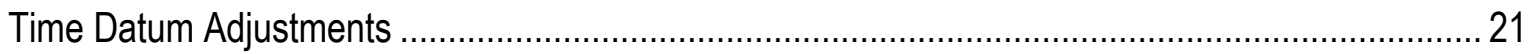

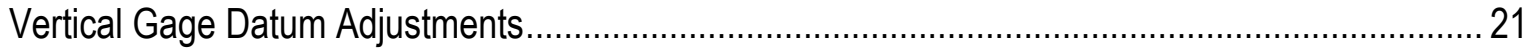

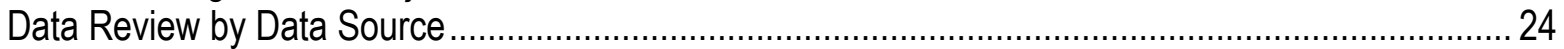

U.S. Geological Survey (USGS) ...................................................................................... 24

Oregon Water Resources Department (OWRD) ................................................................... 25

National Oceanic and Atmospheric Administration (NOAA) …………..................................... 25

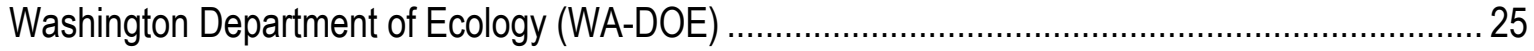

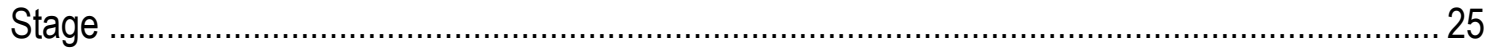

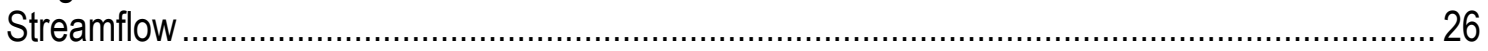

Pacific Northwest National Laboratory (PNNL) ..................................................................... 27

Portland State University (PSU) ………………………………………………….... 27

U.S. Army Corps of Engineers (USACE) ........................................................................ 28

Combining Data from Multiple Sources....................................................................................... 28

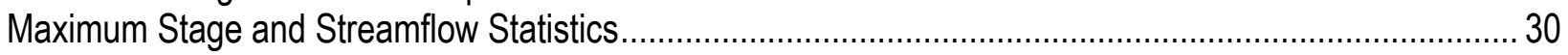

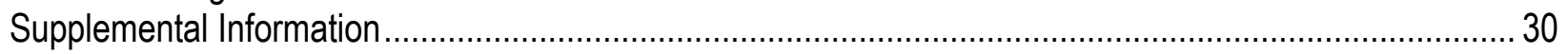

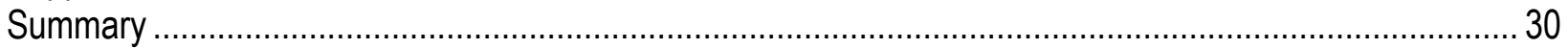

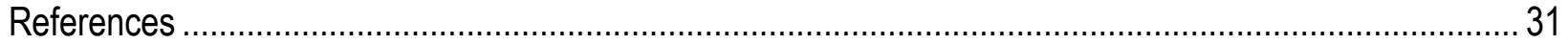

\section{Figures}

Figure 1. Map showing the Lower Columbia River flood profile study area with sub-regions......................33 Figure 2. Map showing the coastal Washington, Oregon, and California sub-region of the Lower Columbia River flood profile study area ..................................................................................................... 34 Figure 3. Map showing the Lower Columbia River Estuary sub-region of the Lower Columbia River flood profile study area, Washington and Oregon ................................................................................ 35 Figure 4. Map showing the Upper Columbia River Estuary sub-region of the Lower Columbia River flood profile study area, Washington and Oregon

Figure 5. Map showing the Longview to Portland sub-region of the Lower Columbia River flood profile study area, Washington and Oregon

Figure 6. Map showing the Willamette River Coastal sub-region of the Lower Columbia River flood profile study area, Oregon 
Figure 7. Map showing the Willamette River Cascades sub-region of the Lower Columbia River flood profile study area, Washington and Oregon

Figure 8. Map showing Troutdale to The Dalles sub-region of the Lower Columbia River flood profile study area, Washington and Oregon.... 40

Figure 9. Graphs showing elevation data that included an example of erroneous data $(A)$ and the edited dataset with the erroneous data removed (B), St. Helens, Oregon. May 2000.

\section{Tables}

Table 1. HEC-DSSVue database file pathname parts and descriptions ................................................ 2

Table 2. Three examples of HEC-DSSVue database file pathnames, following a /A/B/C/D/E/F format ....... 3

Table 3. Abbreviations and detailed explanations for the HEC-DSSVue database pathname parts ............ 3

Table 4. U.S. Geological Survey gaging station list for data compiled in this report ................................. 6

Table 5. Historical paper rating tables that were recreated within the internal U.S. Geological Survey

database to expand and determine instantaneous discharge for flooding events.................................... 10

Table 6. Summary of U.S. Geological Survey historic flood data compiled in this report .........................11

Table 7. Oregon Water Resources Department gaging station list for data compiled in this report........... 13

Table 8. National Oceanic and Atmospheric Administration gaging station list for data compiled in

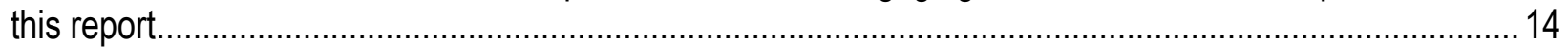

Table 9. Washington Department of Ecology gaging station list for data compiled in this report............... 15

Table 10. Pacific Northwest National Laboratory gaging station list for data compiled in this report .......... 16

Table 11. Portland State University gaging station list for data compiled in this report ............................ 17

Table 12. U.S. Army Corps of Engineers gaging station list for data compiled in this report..................... 19

Table 13. U.S. Army Corps of Engineers post-flood report data compiled for this report ........................ 19

Table 14. Conversion values used to adjust gage datums to North American Vertical Datum of $1988 \ldots . . .22$

Table 15. Staff gage locations over time in Portland, Oregon ............................................................... 27

Table 16. Gaging stations where data were combined from a secondary data source to extend the record

or fill in some missing time periods .................................................................................................... 28

Table 17. Current Oregon Water Resources Department gaging stations that were previously run by U.S.

Geological Survey 30

\section{Conversion Factors}

U.S. customary units to International System of Units

\begin{tabular}{lccc}
\hline & By & To obtain \\
\hline & Length & & \\
\hline foot $(\mathrm{ft})$ & 0.3048 & meter $(\mathrm{m})$ & \\
\hline
\end{tabular}

\section{Datums}

Vertical coordinate information is referenced to various vertical reference datums and primarily to the North American Vertical Datum of 1988 (NAVD 88).

Horizontal coordinate information is referenced to the North American Datum of 1983 (NAD 83).

Elevation, as used in this report, refers to distance above the vertical datum. 


\section{Abbreviations}

$\begin{array}{ll}\text { CRD } & \text { Columbia River Datum } \\ \text { GMT } & \text { Greenwich Mean Time } \\ \text { LCR } & \text { Lower Columbia River } \\ \text { NFDA } & \text { National Federal Data Archive } \\ \text { NOAA } & \text { National Oceanic and Atmospheric Administration } \\ \text { NWIS } & \text { National Water Information System } \\ \text { OWRD } & \text { Oregon Water Resources Department } \\ \text { PDT } & \text { Pacific Daylight Time } \\ \text { PNNL } & \text { Pacific Northwest National Laboratory } \\ \text { PST } & \text { Pacific standard time } \\ \text { PSU } & \text { Portland State University } \\ \text { STND } & \text { Station Datum } \\ \text { USACE } & \text { U.S. Army Corps of Engineers } \\ \text { USGS } & \text { U.S. Geological Survey } \\ \text { WA-DOE } & \text { Washington Department of Ecology }\end{array}$




\title{
Historical Streamflow and Stage Data Compilation for the Lower Columbia River, Pacific Northwest
}

\author{
By Carrie L. Boudreau, Marc A. Stewart, and Adam J. Stonewall
}

\begin{abstract}
The U.S. Geological Survey (USGS) mined data from a variety of national and state agencies including USGS, Oregon Water Resources Department, National Oceanic and Atmospheric Administration, Washington Department of Ecology, Pacific Northwest National Laboratory, Portland State University, and U.S. Army Corps of Engineers (USACE). A comprehensive dataset of streamflow, stage, and tidal elevations for the Lower Columbia River basin was compiled. Data were compiled from gaging stations in Oregon and Washington along the Columbia River from Astoria to The Dalles and along the Willamette River from Salem to Portland. Tidal gages along the Washington, Oregon, and California coasts were also compiled. Seasonal maximum values were calculated for both streamflow and stage for the winter (November-March) and spring (April-July) flow seasons, as well as for the full water year when underlying data were available. The aggregated datasets are available at https://doi.org/10.5066/P9R6RT0Z.
\end{abstract}

\section{Introduction}

The U.S. Geological Survey (USGS) supported the U.S. Army Corps of Engineers in compilation of streamflow and stage data along with quality assurance of acquired data to be used by USACE in a reanalysis of the current flood profiles for the Lower Columbia River (LCR) basin. Flood profiles, also known as "stage-frequency curves," are defined as the annual probability the water level at a given river mile will exceed a specific elevation. The use of accurate flood profiles is important for floodplain management and levee risk assessments. The flood profiles currently used for the Lower Columbia and Willamette Rivers were first developed in 1990 as part of the Columbia River and Tributaries Study 1963 (U.S. Army Corps of Engineers, 1991).

\section{Purpose and Scope}

The purpose of this study was to compile an up-to-date dataset of daily mean streamflow, annual peak streamflow, stage, and tidal elevations that will allow for USACE to reanalyze flood profiles and update estimates of uncertainty. The updated profiles will support the mission of the U.S. Army Corps of Engineers (USACE) and other public agencies in the study area involved with the mitigation of flood impacts. All findings are summarized in this report with data published in Boudreau and others (2021). Additionally, three USGS stations were analyzed to provide additional information for future hydrologic modeling efforts in the study area. 


\section{Study Area}

The LCR basin includes all watersheds draining into the Columbia River from its mouth to downstream of the Bonneville Dam (river mile 146). While much of the study area was in the LCR basin, a few additional areas, upstream of Bonneville Dam to the Dalles along with some tidal stations along the California, Oregon, and Washington coasts, were included. Tidal stations were included for this study in order to look at potential effects from tides and surges on the interior gages. The study area (fig. 1) includes the Columbia River from Astoria to The Dalles, the Willamette River from Salem to Portland, and portions of the California, Oregon, and Washington coasts. For easier viewing, the study area (fig. 1) was divided into sub-regions, which included gages from all data sources for the designated regions: Coastal Washington and Oregon (fig. 2), Lower Columbia River Estuary (fig. 3), Upper Columbia River Estuary (fig. 4), Longview to Portland (fig. 5), Willamette River Coastal (fig. 6), Willamette River Cascades (fig. 7), and Troutdale to The Dalles (fig. 8) gages. Figures 2 through 8 depict the individual subregions.

Figures 1-8. Figures 1-8 are located at this back of the report.

\section{Methodology}

Streamflow data were gathered as daily-mean streamflow and annual peak streamflow along the Columbia River from The Dalles to Astoria and along the Willamette River from Salem to Portland. Data were not collected from stations that did not contain sufficient period of record length or were not in locations needed for future modeling efforts. When available, finerresolution streamflow data (sub-daily) were collected for a selection of historic flooding events that occurred on December 1933, December 1964, January 1974, February 1982, February 1986, February 1996, January 1997, and January 2006. Stage data were gathered at a finer resolution (sub-daily) from available locations on the Columbia River below Bonneville Dam and along the Willamette River below Willamette Falls. Hourly tidal data were gathered from sites along the Oregon and Washington coasts as well as from sites on the Columbia River from Vancouver, Washington, to Astoria, Oregon (fig. 2).

All data were compiled in a HEC-DSSVue (U.S. Army Corps of Engineers, 2018a) database file. HEC-DSSVue is a java-based program allowing the user to both visualize and manipulate data in the database file format (.dss). Each dataset is saved in HEC-DSSVue with an associated pathname that follows the format $/ \mathrm{A} / \mathrm{B} / \mathrm{C} / \mathrm{D} / \mathrm{E} / \mathrm{F}$. The various parts, $\mathrm{A}-\mathrm{F}$, are described in more detail in table 1, along with pathname examples in table 2.

Table 1. HEC-DSSVue database file pathname parts and descriptions.

\begin{tabular}{cl}
\hline Part & \multicolumn{1}{c}{ Description } \\
\hline A & Project, river, or basin name \\
B & Location or station identifier \\
C & Data parameter \\
D & Time frame of data \\
E & Time interval \\
F & Additional user-defined information \\
\hline
\end{tabular}


Table 2. Three examples of HEC-DSSVue database file pathnames, following a /A/B/C/D/E/F format.

[/A/B/C/D/E/F, Project river or basin name/Location or station identifier/Data parameter/Time frame of data/Time interval/Additional user-defined information]

\begin{tabular}{cc}
\hline Example & File pathname \\
\hline \multirow{2}{*}{1} & /WILLAMETTE RIVER AT NEWBERG, OR/14197900/FLOW-DAILY MEAN/01JAN2001- \\
& 01JAN2018/1DAY/USGS-NWIS/ \\
2 & /WILLAMETTE RIVER AT NEWBERG, OR/14197900/ELEV-NAVD88/01OCT2001- \\
3 & 01MAR2018/IR-MONTH/USGS-AQ-CALCULATED/ \\
3 & OWILLAMETTE RIVER AT NEWBERG, OR/14197900/FLOW-2006/01DEC2005- \\
\hline
\end{tabular}

Pathname parts A or B could be blank if a river name or station identifier/location were not available from the data source. Names were not added to these records in order to avoid future confusion when referencing the original data. For part $\mathrm{C}$, the data parameter, see table 3 for explanations of the abbreviations used and their expanded descriptions. In some instances, part $\mathrm{D}$, the data time range, could differ slightly in the pathname versus the actual dataset if the original data file was edited after upload. The time interval, part E, abbreviations are explained in table $3 B$. Part F, the additional user-defined information, was used to note the source where the data were retrieved from for this study. Table 3 describes the abbreviated part F sources with an expanded description.

Table 3. Abbreviations and detailed explanations for the HEC-DSSVue database pathname parts.

[Abbreviations: NAVD 88, North American Vertical Datum of 1988; USACE, U.S. Army Corps of Engineers]

\begin{tabular}{|c|c|}
\hline Abbreviation & Description \\
\hline \multicolumn{2}{|r|}{ HEC-DSSVue pathname part C: Data parameter } \\
\hline ELEV-NAVD88 & Elevation in NAVD 88 \\
\hline ELEV-NAVD88-MAX & Daily maximum elevation in NAVD 88 \\
\hline ELEV-NAVD88-MEAN & Daily mean elevation in NAVD 88 \\
\hline ELEV-NAVD88-MIN & Daily minimum elevation in NAVD 88 \\
\hline ELEV-STND & Elevation in Station Datum (an arbitrarily established fixed elevation) \\
\hline ELEV-TAILWATER & Elevation tailwater (below dam structures) \\
\hline FLOW & Streamflow (digitized from paper file post-flood reports) \\
\hline FLOW-1933 & Streamflow from the 1933 flooding event \\
\hline FLOW-1964 & Streamflow from the 1964 flooding event \\
\hline FLOW-1974 & Streamflow from the 1974 flooding event \\
\hline FLOW-1982 & Streamflow from the 1982 flooding event \\
\hline FLOW-1986 & Streamflow from the 1986 flooding event \\
\hline FLOW-1996 & Streamflow from the 1996 flooding event \\
\hline FLOW-1997 & Streamflow from the 1997 flooding event \\
\hline FLOW-2006 & Streamflow from the 2006 flooding event \\
\hline FLOW-ANNUAL PEAK & Annual peak streamflow \\
\hline FLOW-DAILY MEAN & Daily mean streamflow \\
\hline FLOW-OUT & $\begin{array}{l}\text { Calculated streamflow output below structures (calculated by USACE } \\
\text { based on hydraulic tables for gate openings) }\end{array}$ \\
\hline STAGE & Stage (local datum) \\
\hline STAGE-DAILY MAX & Daily maximum stage \\
\hline STAGE-DAILY MEAN & Daily mean stage \\
\hline STAGE-DAILY MIN & Daily minimum stage \\
\hline STAGE-EVENT MAX & Event maximum stage values \\
\hline
\end{tabular}




\begin{tabular}{|c|c|}
\hline Abbreviation & Description \\
\hline STAGE-SPING ANNUAL MAX & Spring peak stage values (digitized from USACE drawings) \\
\hline STAGE-WINTER ANNUAL MAX & Winter peak stage values (digitized from USACE drawings) \\
\hline \multicolumn{2}{|c|}{ HEC-DSSVue pathname part E: Time Interval } \\
\hline $15 \mathrm{MIN}$ & 15-minute time stamp interval \\
\hline 1DAY & Daily time stamp interval \\
\hline 1YEAR & Annual time stamp interval \\
\hline 2HOUR & 2-hour time stamp interval \\
\hline $30 \mathrm{MIN}$ & 30-minute time stamp interval \\
\hline IR-CENTURY & $\begin{array}{l}\text { Irregular, varying time interval on a century time block. Associated only } \\
\text { with the Annual Peak Streamflow datasets. }\end{array}$ \\
\hline IR-MONTH & Irregular, varying time interval on a month time block. \\
\hline IR-YEAR & Irregular, varying time interval on a year time block. \\
\hline $15 \mathrm{MIN}$ & 15-minute time stamp interval \\
\hline \multicolumn{2}{|r|}{ HEC-DSSVue pathname part F: Data Source } \\
\hline 1943 POST-FLOOD REPORT & Digitized paper files from the 1943 USACE post-flood report \\
\hline 1948 POST-FLOOD REPORT & Digitized paper files from the 1948 USACE post-flood report \\
\hline 1954 POST-FLOOD REPORT & Digitized paper files from the 1954 USACE post-flood report \\
\hline 1956 POST-FLOOD REPORT & Digitized paper files from the 1956 USACE post-flood report \\
\hline 1961 POST-FLOOD REPORT & Digitized paper files from the 1961 USACE post-flood report \\
\hline 1964 POST-FLOOD REPORT & Digitized paper files from the 1964 USACE post-flood report \\
\hline 1965 POST-FLOOD REPORT & Digitized paper files from the 1965 USACE post-flood report \\
\hline 1974 POST-FLOOD REPORT & Digitized paper files from the 1974 USACE post-flood report \\
\hline 1996 POST FLOOD REPORT & Digitized paper files from the 1996 USACE post-flood report \\
\hline CL -03-112, CL -03-116 & Digitized data from USACE drawings CL $-03-112$ and CL $-03-116$ \\
\hline COMBINED-DQ-NOAA & $\begin{array}{l}\text { Data files combined with USACE Dataquery data supplementing NOAA } \\
\text { data }\end{array}$ \\
\hline COMBINED-DQ-USGS & $\begin{array}{l}\text { Data files combined with USACE Dataquery data supplementing USGS } \\
\text { data }\end{array}$ \\
\hline $\begin{array}{l}\text { COMBINED-DQ-USGS- } \\
\text { CALCULATED }\end{array}$ & $\begin{array}{l}\text { Data files combined with USACE Dataquery data supplementing USGS } \\
\text { data, converted to elevation in NAVD } 88\end{array}$ \\
\hline DATAQUERY & Data files supplied by USACE Dataquery database \\
\hline DATAQUERY-CALCULATED & $\begin{array}{l}\text { Data files supplied by USACE Dataquery database, converted to NAVD } \\
88\end{array}$ \\
\hline NOAA & National Oceanic and Atmospheric Administration \\
\hline OWRD & Oregon Water Resources department \\
\hline PNNL & Pacific Northwest National Laboratory \\
\hline PSU & Portland State University \\
\hline PSU-NOAA & NOAA data retrieved by Portland State University \\
\hline PSU-NOAA-CORRECTED & Portland State University determined datum correction to NOAA dataset \\
\hline USACE POST-FLOOD REPORTS & Digitized paper files from various USACE post-flood reports \\
\hline USGS-AQ-CALCULATED & $\begin{array}{l}\text { USGS data retrieved from the internal NWIS database, converted to } \\
\text { elevation in NAVD } 88\end{array}$ \\
\hline USGS-ARCHIVES & USGS data retrieved from the National Federal Data Archive \\
\hline USGS-NWIS & USGS data retrieved from the National Water Information System \\
\hline USGS-NWIS-CALCULATED & $\begin{array}{l}\text { USGS stage data retrieved from the National Water Information System, } \\
\text { converted to elevation in NAVD } 88\end{array}$ \\
\hline USGS-OWRD & Stations previously operated by USGS and currently operated by OWRD \\
\hline WA-DOE & Washington Department of Ecology \\
\hline
\end{tabular}




\section{Data Sources}

This section summarizes sources from which data were compiled. Gages were not identified as being tidally influenced or regulated during this data compilation.

\section{United States Geological Survey (USGS)}

Data from 65 USGS gages were compiled for this study (table 4; figs. 4, 5, 6, 7, and 8). Multiple sources were used when compiling data from USGS stations. The USGS National Water Information System (NWIS) website was used for both historical and recent readily available data (U.S. Geological Survey, 2018). The USGS internal NWIS database was used to download stage data which has historically not been available on NWIS. Historical data that were not available in NWIS or electronic format were requested from the National Federal Data Archive (NFDA). An inventory of available data was searched, and a request made to the NFDA for temporary use of the archived records of interest. These records included pre-digital, analog recordings of gage height, such as mechanical stage recorders, paper copies of daily flow values, historic gage documentation, and paper copies of stage-discharge rating tables. Some historical stage-discharge rating tables (table 5) were recreated in the internal USGS database so they could be expanded to determine streamflow from historic stage data. For the historic flooding events of interest, 45 USGS gages had at least 1 flooding event where finer resolution (sub-daily) data were retrieved. Table 6 details the USGS sites and corresponding flooding event dates compiled. All datasets were compiled in Pacific standard time (PST). The gage datum used for the compiled stage data varied. Recorded datums were Columbia River Datum (CRD), National Geodetic Vertical Datum of 1929 (NGVD 29), USGS datum, or North American Vertical Datum of 1988 (NAVD 88). 
Table 4. U.S. Geological Survey gaging station list for data compiled in this report.

[Map no. (number) may not be in numerical order based on how stations were grouped by sources during map creation. Period of record may not be continuous. Abbreviations: Ab, above; Bl, below; Cr, Creek; Nr, near; OR, Oregon; R, river; WA, Washington]

\begin{tabular}{|c|c|c|c|c|c|c|}
\hline $\begin{array}{c}\text { Map } \\
\text { no. }\end{array}$ & $\begin{array}{l}\text { Station } \\
\text { identifier }\end{array}$ & Station name & $\begin{array}{l}\text { Latitude } \\
\text { (decimal } \\
\text { degrees) }\end{array}$ & $\begin{array}{l}\text { Longitude } \\
\text { (decimal } \\
\text { degrees) }\end{array}$ & Period of record & Data compiled \\
\hline 1 & 14105700 & $\begin{array}{l}\text { Columbia River at The Dalles, } \\
\text { OR }\end{array}$ & 45.60827778 & -121.1899167 & $\begin{array}{l}\text { June 1, 1878-March } \\
5,2018^{1}\end{array}$ & $\begin{array}{l}\text { Daily mean streamflow, annual peak } \\
\text { streamflow }\end{array}$ \\
\hline 2 & 14113000 & Klickitat River Near Pitt, WA & 45.75651098 & -121.2100714 & $\begin{array}{l}\text { July 1, 1909-March 5, } \\
\quad 2018^{1}\end{array}$ & $\begin{array}{l}\text { Daily mean streamflow, annual peak } \\
\text { streamflow }\end{array}$ \\
\hline 3 & 14113200 & Mosier Creek Near Mosier, OR & 45.649008 & -121.3772951 & $\begin{array}{l}\text { April 17, 1963-March } \\
\quad 5,2018^{1}\end{array}$ & $\begin{array}{l}\text { Daily mean streamflow, annual peak } \\
\text { streamflow }\end{array}$ \\
\hline 4 & 14120000 & $\begin{array}{l}\text { Hood River at Tucker Bridge, } \\
\text { Near Hood River, OR }\end{array}$ & 45.6545 & -121.5488 & $\begin{array}{l}\text { October 20, 1897- } \\
\quad \text { March 5, } 2018^{1}\end{array}$ & $\begin{array}{l}\text { Daily mean streamflow, annual peak } \\
\text { streamflow }\end{array}$ \\
\hline 5 & 14123500 & $\begin{array}{l}\text { White Salmon River Near } \\
\text { Underwood, WA }\end{array}$ & 45.7520637 & -121.5270176 & $\begin{array}{l}\text { November 1, 1912- } \\
\quad \text { March 5, 2018 }\end{array}$ & $\begin{array}{l}\text { Daily mean streamflow, annual peak } \\
\text { streamflow }\end{array}$ \\
\hline 6 & 14125500 & $\begin{array}{l}\text { Little White Salmon River Near } \\
\text { Cook, WA }\end{array}$ & 45.72345147 & -121.6339631 & $\begin{array}{l}\text { October 1, 1956- } \\
\text { October 5, } 1977\end{array}$ & $\begin{array}{l}\text { Daily mean streamflow, annual peak } \\
\text { streamflow }\end{array}$ \\
\hline 7 & 14128500 & Wind River Near Carson, WA & 45.7267837 & -121.794799 & $\begin{array}{l}\text { October } 1,1934- \\
\text { October } 14,1980\end{array}$ & $\begin{array}{l}\text { Daily mean streamflow, annual peak } \\
\text { streamflow }\end{array}$ \\
\hline 52 & 14128870 & $\begin{array}{r}\text { Columbia River Below } \\
\text { Bonneville Dam, OR }\end{array}$ & 45.63305556 & -121.9608333 & $\begin{array}{l}\text { September 30, 1986- } \\
\quad \text { April 16, } 2018^{\mathrm{b}}\end{array}$ & Stage \\
\hline 8 & 14128910 & $\begin{array}{l}\text { Columbia River at Warrendale, } \\
\text { OR }\end{array}$ & 45.6123396 & -122.0275838 & $\begin{array}{l}\text { October 27, 1971- } \\
\quad \text { September 29, } 1987\end{array}$ & Stage (daily max, min, mean) \\
\hline 9 & 14137000 & Sandy River Near Marmot, OR & 45.3995642 & -122.1373068 & $\begin{array}{l}\text { September 1, 1911- } \\
\quad \text { March 5, 2018 }\end{array}$ & $\begin{array}{l}\text { Daily mean streamflow, annual peak } \\
\text { streamflow }\end{array}$ \\
\hline 10 & 14141500 & $\begin{array}{l}\text { Little Sandy River Near Bull Run, } \\
\text { OR }\end{array}$ & 45.41539777 & -122.1714746 & $\begin{array}{l}\text { May 1, 1911-March } \\
\quad 5,2018^{1}\end{array}$ & $\begin{array}{l}\text { Daily mean streamflow, annual peak } \\
\text { streamflow }\end{array}$ \\
\hline 11 & 14142500 & $\begin{array}{l}\text { Sandy River Blw Bull Run River, } \\
\text { Nr Bull Run, OR }\end{array}$ & 45.4490094 & -122.2450885 & $\begin{array}{l}\text { October 1, 1910- } \\
\quad \text { March 5, 2018 }\end{array}$ & $\begin{array}{l}\text { Daily mean streamflow, annual peak } \\
\text { streamflow }\end{array}$ \\
\hline 12 & 14143500 & $\begin{array}{l}\text { Washougal River Near } \\
\text { Washougal, WA }\end{array}$ & 45.6231733 & -122.2975912 & $\begin{array}{l}\text { October 1, 1944- } \\
\text { October 29, } 1981\end{array}$ & $\begin{array}{l}\text { Daily mean streamflow, annual peak } \\
\text { streamflow }\end{array}$ \\
\hline 13 & 14144000 & $\begin{array}{l}\text { Little Washougal River Near } \\
\text { Washougal, WA }\end{array}$ & 45.614007 & -122.358425 & $\begin{array}{l}\text { July } 1,1951- \\
\quad \text { November } 30,1955\end{array}$ & $\begin{array}{l}\text { Daily mean streamflow, annual peak } \\
\text { streamflow }\end{array}$ \\
\hline 14 & 14142800 & Beaver Creek at Troutdale, OR & 45.5192866 & -122.3889798 & $\begin{array}{l}\text { October 1, 1999- } \\
\quad \text { March 5, } 2018^{1}\end{array}$ & $\begin{array}{l}\text { Daily mean streamflow, annual peak } \\
\text { streamflow }\end{array}$ \\
\hline
\end{tabular}




\begin{tabular}{|c|c|c|c|c|c|c|}
\hline $\begin{array}{c}\text { Map } \\
\text { no. }\end{array}$ & $\begin{array}{l}\text { Station } \\
\text { identifier }\end{array}$ & Station name & $\begin{array}{l}\text { Latitude } \\
\text { (decimal } \\
\text { degrees) }\end{array}$ & $\begin{array}{l}\text { Longitude } \\
\text { (decimal } \\
\text { degrees) }\end{array}$ & Period of record & Data compiled \\
\hline 58 & 14144700 & $\begin{array}{l}\text { Columbia River at Vancouver, } \\
\text { WA }\end{array}$ & 45.62067265 & -122.6734306 & $\begin{array}{l}\text { October 1, 1963- } \\
\quad \text { March 5, 2018 }\end{array}$ & Daily mean streamflow, stage \\
\hline 15 & 14191000 & Willamette River at Salem, OR & 44.9442863 & -123.0428742 & $\begin{array}{l}\text { October 1, 1909- } \\
\quad \text { March 5, 2018 }\end{array}$ & $\begin{array}{l}\text { Daily mean streamflow, annual peak } \\
\text { streamflow }\end{array}$ \\
\hline 68 & 14192500 & S Yamhill R Nr Willamina, OR & 45.04742 & -123.504292 & $\begin{array}{l}\text { May 1, 1934- } \\
\text { September 29, } 1993\end{array}$ & $\begin{array}{l}\text { Daily mean streamflow, annual peak } \\
\text { streamflow }\end{array}$ \\
\hline 69 & 14193000 & Willamina Cr Nr Willamina, OR & 45.143242 & -123.494639 & $\begin{array}{l}\text { June } 1,1934-\text { October } \\
29,1991\end{array}$ & $\begin{array}{l}\text { Daily mean streamflow, annual peak } \\
\text { streamflow }\end{array}$ \\
\hline 16 & 14194150 & $\begin{array}{l}\text { South Yamhill River at } \\
\text { Mcminnville, OR }\end{array}$ & 45.2056719 & -123.1826047 & $\begin{array}{l}\text { October 1, 1994- } \\
\quad \text { March 5, 2018 }\end{array}$ & $\begin{array}{l}\text { Daily mean streamflow, annual peak } \\
\text { streamflow }\end{array}$ \\
\hline 70 & 14194300 & N Yamhill R Nr Fairdale, OR & 45.364959 & -123.3793 & $\begin{array}{l}\text { October } 1,1958- \\
\text { October } 29,1991\end{array}$ & $\begin{array}{l}\text { Daily mean streamflow, annual peak } \\
\text { streamflow }\end{array}$ \\
\hline 17 & 14198400 & Bull Creek Near Wilhoit, OR & 44.9609 & -122.385 & $\begin{array}{l}\text { March 31, 1993- } \\
\quad \text { March 5, 2018 }\end{array}$ & $\begin{array}{l}\text { Daily mean streamflow, annual peak } \\
\text { streamflow }\end{array}$ \\
\hline 18 & 14198500 & Molalla R Ab Pc Nr Wilhoit, OR & 45.00956696 & -122.480362 & $\begin{array}{l}\text { October 1, 1935- } \\
\quad \text { September 29, } 1993\end{array}$ & $\begin{array}{l}\text { Daily mean streamflow, annual peak } \\
\text { streamflow }\end{array}$ \\
\hline 19 & 14199000 & Molalla River Near Molalla, OR & 45.11928929 & -122.534531 & $\begin{array}{l}\text { October } 1,1905- \\
\text { October } 29,1951\end{array}$ & $\begin{array}{l}\text { Daily mean streamflow, annual peak } \\
\text { streamflow }\end{array}$ \\
\hline 20 & 14197000 & North Yamhill R at Pike, OR & 45.36928017 & -123.2553867 & $\begin{array}{l}\text { October 1, 1948- } \\
\quad \text { September } 29,1973\end{array}$ & $\begin{array}{l}\text { Daily mean streamflow, annual peak } \\
\text { streamflow }\end{array}$ \\
\hline 21 & 14197900 & Willamette River at Newberg, OR & 45.2845625 & -122.9614893 & $\begin{array}{l}\text { October 1, 2001- } \\
\quad \text { March 5, 2018 }\end{array}$ & $\begin{array}{l}\text { Daily mean streamflow, annual peak } \\
\text { streamflow, Stage }\end{array}$ \\
\hline 22 & 14198000 & $\begin{array}{l}\text { Willamette River at Wilsonville, } \\
\text { OR }\end{array}$ & 45.2990086 & -122.7512055 & $\begin{array}{l}\text { October } 1,1948-\text { July } \\
\quad 31,1973\end{array}$ & $\begin{array}{l}\text { Daily mean streamflow, annual peak } \\
\text { streamflow }\end{array}$ \\
\hline 23 & 14200000 & Molalla River Near Canby, OR & 45.24428767 & -122.687314 & $\begin{array}{l}\text { August 1, 1928- } \\
\quad \text { March 5, 2018 }\end{array}$ & $\begin{array}{l}\text { Daily mean streamflow, annual peak } \\
\text { streamflow }\end{array}$ \\
\hline 24 & 14200100 & Drift Creek Near Silverton, OR & 44.97680556 & -122.830111 & $\begin{array}{l}\text { May 21, 2014- } \\
\quad \text { February 5, } 2018\end{array}$ & $\begin{array}{l}\text { Daily mean streamflow, annual peak } \\
\text { streamflow }\end{array}$ \\
\hline 25 & 14200300 & Silver Creek at Silverton, OR & 45.0092878 & -122.7887014 & $\begin{array}{l}\text { October 1, 1963- } \\
\quad \text { February 5, } 2018\end{array}$ & $\begin{array}{l}\text { Daily mean streamflow, annual peak } \\
\text { streamflow }\end{array}$ \\
\hline 26 & 14200700 & Abiqua Creek at Silverton, OR & 45.03145 & -122.7908306 & $\begin{array}{l}\text { June } 12,2014-\text { March } \\
\quad 6,2018^{1}\end{array}$ & $\begin{array}{l}\text { Daily mean streamflow, annual peak } \\
\text { streamflow }\end{array}$ \\
\hline 27 & 14201500 & Butte Creek at Monitor, OR & 45.1015099 & -122.7462019 & $\begin{array}{l}\text { January 1, 1936- } \\
\quad \text { March 5, } 2018^{1}\end{array}$ & $\begin{array}{l}\text { Daily mean streamflow, annual peak } \\
\text { streamflow }\end{array}$ \\
\hline 28 & 14201300 & Zollner Creek Near Mt Angel, OR & 45.10039816 & -122.8217596 & $\begin{array}{l}\text { September 1, 1993- } \\
\text { March 6, 2018 }\end{array}$ & $\begin{array}{l}\text { Daily mean streamflow, annual peak } \\
\text { streamflow }\end{array}$ \\
\hline
\end{tabular}




\begin{tabular}{|c|c|c|c|c|c|c|}
\hline $\begin{array}{l}\text { Map } \\
\text { no. }\end{array}$ & $\begin{array}{l}\text { Station } \\
\text { identifier }\end{array}$ & Station name & $\begin{array}{l}\text { Latitude } \\
\text { (decimal } \\
\text { degrees) }\end{array}$ & $\begin{array}{l}\text { Longitude } \\
\text { (decimal } \\
\text { degrees) }\end{array}$ & Period of record & Data compiled \\
\hline 29 & 14201340 & $\begin{array}{l}\text { Pudding River Near Woodburn, } \\
\text { OR }\end{array}$ & 45.15123137 & -122.80426 & $\begin{array}{l}\text { October } 1,1997- \\
\quad \text { March } 6,2018^{1}\end{array}$ & $\begin{array}{l}\text { Daily mean streamflow, annual peak } \\
\text { streamflow }\end{array}$ \\
\hline 30 & 14202000 & Pudding River at Aurora, OR & 45.23317586 & -122.7500933 & $\begin{array}{l}\text { October 1, 1928- } \\
\quad \text { March 6, 2018 }\end{array}$ & $\begin{array}{l}\text { Daily mean streamflow, annual peak } \\
\text { streamflow }\end{array}$ \\
\hline 31 & 14202500 & Tualatin R Nr Gaston, OR & 45.4366684 & -123.1688843 & $\begin{array}{l}\text { October } 1,1940- \\
\quad \text { February } 25,1985\end{array}$ & $\begin{array}{l}\text { Daily mean streamflow, annual peak } \\
\text { streamflow }\end{array}$ \\
\hline 71 & 14202850 & $\begin{array}{l}\text { Scoggins Cr Ab Henry Hagg Lake } \\
\text { Nr Gaston, OR }\end{array}$ & 45.499942 & -123.251188 & $\begin{array}{l}\text { October } 1,1972- \\
\quad \text { October } 29,1976\end{array}$ & $\begin{array}{l}\text { Daily mean streamflow, annual peak } \\
\text { streamflow }\end{array}$ \\
\hline 72 & 14202920 & Sain Cr Nr Gaston, OR & 45.480803 & -123.245886 & $\begin{array}{l}\text { October } 1,1972- \\
\text { October 29, } 1976\end{array}$ & $\begin{array}{l}\text { Daily mean streamflow, annual peak } \\
\text { streamflow }\end{array}$ \\
\hline 73 & 14202980 & $\begin{array}{l}\text { Scoggins Cr Bl Henry Hagg Lake } \\
\text { Nr Gaston, OR }\end{array}$ & 45.471756 & -123.197467 & $\begin{array}{l}\text { January } 1,1975- \\
\text { October } 1,2006\end{array}$ & $\begin{array}{l}\text { Daily mean streamflow, annual peak } \\
\text { streamflow }\end{array}$ \\
\hline 32 & 14203500 & Tualatin River Near Dilley, OR & 45.4748365 & -123.124274 & $\begin{array}{l}\text { October 1, 1939- } \\
\quad \text { March 6, 2018 }\end{array}$ & $\begin{array}{l}\text { Daily mean streamflow, annual peak } \\
\text { streamflow }\end{array}$ \\
\hline 33 & 14205400 & $\begin{array}{l}\text { East Fork Dairy Creek Near } \\
\text { Meacham Corner, OR }\end{array}$ & 45.68233568 & -123.069553 & $\begin{array}{l}\text { May 8, 2002-March } \\
\quad 6,2018^{1}\end{array}$ & $\begin{array}{l}\text { Daily mean streamflow, annual peak } \\
\text { streamflow }\end{array}$ \\
\hline 74 & 14206500 & Tualatin River at Farmington, OR & 45.44983918 & -122.9512138 & $\begin{array}{l}\text { October } 1,1939- \\
\text { October 29, } 1976\end{array}$ & $\begin{array}{l}\text { Daily mean streamflow, annual peak } \\
\text { streamflow }\end{array}$ \\
\hline 34 & 14206950 & Fanno Creek at Durham, OR & 45.403452 & -122.7548185 & $\begin{array}{l}\text { October 1, 1993- } \\
\quad \text { March 6, 2018 }\end{array}$ & $\begin{array}{l}\text { Daily mean streamflow, annual peak } \\
\text { streamflow }\end{array}$ \\
\hline 35 & 14207500 & Tualatin River at West Linn, OR & 45.35067559 & -122.6762044 & $\begin{array}{l}\text { August 1, 1928- } \\
\quad \text { March 6, 2018 }\end{array}$ & $\begin{array}{l}\text { Daily mean streamflow, annual peak } \\
\text { streamflow }\end{array}$ \\
\hline 54 & 14207740 & $\begin{array}{l}\text { Willamette River Above Falls, at } \\
\text { Oregon City, OR }\end{array}$ & 45.348454 & -122.6200918 & $\begin{array}{l}\text { September } 30,1986- \\
3 / 29 / 2018^{1}\end{array}$ & Stage \\
\hline 55 & 14207770 & $\begin{array}{l}\text { Willamette River Below Falls, at } \\
\text { Oregon City, OR }\end{array}$ & 45.35762066 & -122.610925 & $\begin{array}{l}\text { September 30, 1988- } \\
\quad 6 / 18 / 2018^{1}\end{array}$ & Stage \\
\hline 36 & 14211010 & $\begin{array}{l}\text { Clackamas River Near Oregon } \\
\text { City, OR }\end{array}$ & 45.3792874 & -122.5773134 & $\begin{array}{l}\text { June } 1,2001-\text { March } \\
\quad 6,2018^{1}\end{array}$ & $\begin{array}{l}\text { Daily mean streamflow, annual peak } \\
\text { streamflow, stage }\end{array}$ \\
\hline 37 & 14210000 & Clackamas River at Estacada, OR & 45.29984346 & -122.3539746 & $\begin{array}{l}\text { April 1, 1908-March } \\
\quad 6,2018^{1}\end{array}$ & $\begin{array}{l}\text { Daily mean streamflow, annual peak } \\
\text { streamflow }\end{array}$ \\
\hline 38 & 14211315 & $\begin{array}{l}\text { Tryon Creek Near Lake Oswego, } \\
\text { OR }\end{array}$ & 45.43067476 & -122.6737057 & $\begin{array}{l}\text { August 1, 2001- } \\
\quad \text { March 6, 2018 }\end{array}$ & $\begin{array}{l}\text { Daily mean streamflow, annual peak } \\
\text { streamflow }\end{array}$ \\
\hline 39 & 14211500 & Johnson Creek at Sycamore, OR & 45.47745556 & -122.5080194 & $\begin{array}{l}\text { October 1, 1940- } \\
\quad \text { March 6, 2018 }\end{array}$ & $\begin{array}{l}\text { Daily mean streamflow, annual peak } \\
\text { streamflow }\end{array}$ \\
\hline 40 & 14211550 & Johnson Creek at Milwaukie, OR & 45.452897 & -122.6431496 & $\begin{array}{l}\text { April 22, 1989-March } \\
\quad 6,2018^{1}\end{array}$ & $\begin{array}{l}\text { Daily mean streamflow, annual peak } \\
\text { streamflow, stage }\end{array}$ \\
\hline
\end{tabular}




\begin{tabular}{|c|c|c|c|c|c|c|}
\hline $\begin{array}{l}\text { Map } \\
\text { no. }\end{array}$ & $\begin{array}{l}\text { Station } \\
\text { identifier }\end{array}$ & Station name & $\begin{array}{l}\text { Latitude } \\
\text { (decimal } \\
\text { degrees) }\end{array}$ & $\begin{array}{l}\text { Longitude } \\
\text { (decimal } \\
\text { degrees) }\end{array}$ & Period of record & Data compiled \\
\hline 56 & 14211720 & Willamette River at Portland, OR & 45.5175 & -122.6691667 & $\begin{array}{l}\text { October 1, 1972- } \\
\quad \text { March 6, 2018 }\end{array}$ & $\begin{array}{l}\text { Daily mean streamflow, annual peak } \\
\text { streamflow, stage }\end{array}$ \\
\hline 41 & 14211814 & $\begin{array}{l}\text { Fairview Creek at Glisan St Near } \\
\text { Gresham, OR }\end{array}$ & 45.52761968 & -122.4487024 & $\begin{array}{l}\text { May 1, 1992-March } \\
\quad 6,2018^{1}\end{array}$ & $\begin{array}{l}\text { Daily mean streamflow, annual peak } \\
\text { streamflow }\end{array}$ \\
\hline 42 & 14211820 & Columbia Slough at Portland, OR & 45.63900515 & -122.763155 & $\begin{array}{l}\text { October } 14,1988- \\
\text { October } 2,2017^{1}\end{array}$ & $\begin{array}{l}\text { Daily mean streamflow, annual peak } \\
\text { streamflow, stage }\end{array}$ \\
\hline 43 & 14211902 & $\begin{array}{l}\text { Burnt Bridge Creek Near Mouth } \\
\text { at Vancouver, WA }\end{array}$ & 45.66122778 & -122.6689866 & $\begin{array}{l}\text { October } 1,1998- \\
11 / 12 / 2012\end{array}$ & $\begin{array}{l}\text { Daily mean streamflow, annual peak } \\
\text { streamflow }\end{array}$ \\
\hline 44 & 14222500 & $\begin{array}{l}\text { East Fork Lewis River Near } \\
\text { Heisson, WA }\end{array}$ & 45.8367809 & -122.4662083 & $\begin{array}{l}\text { October 1, 1929- } \\
\quad \text { March 6, 2018 }\end{array}$ & $\begin{array}{l}\text { Daily mean streamflow, annual peak } \\
\text { streamflow }\end{array}$ \\
\hline 45 & 14220500 & Lewis River at Ariel, WA & 45.9517789 & -122.5639899 & $\begin{array}{l}\text { July 1, 1909-March 6, } \\
2018^{1}\end{array}$ & $\begin{array}{l}\text { Daily mean streamflow, annual peak } \\
\text { streamflow }\end{array}$ \\
\hline 53 & 14222880 & $\begin{array}{l}\text { Columbia River at Columbia City, } \\
\text { OR }\end{array}$ & 45.8945588 & -122.8076038 & $\begin{array}{l}\text { October } 14,1971- \\
\text { December } 1,1981\end{array}$ & Stage (Daily max, min, mean) \\
\hline 46 & 14223000 & Kalama River Near Kalama, WA & 46.01705847 & -122.7323262 & $\begin{array}{l}\text { July } 1,1911-\text { October } \\
\text { 29, } 1932\end{array}$ & $\begin{array}{l}\text { Daily mean streamflow, annual peak } \\
\text { streamflow }\end{array}$ \\
\hline 47 & 14223500 & $\begin{array}{l}\text { Kalama River Below Italian Creek } \\
\text { Near Kalama, WA }\end{array}$ & 46.044836 & -122.8153843 & $\begin{array}{l}\text { October 1, 1946- } \\
\quad \text { September 12, } 1982\end{array}$ & $\begin{array}{l}\text { Daily mean streamflow, annual peak } \\
\text { streamflow }\end{array}$ \\
\hline 57 & 14243000 & $\begin{array}{l}\text { Cowlitz River at Castle Rock, } \\
\text { WA }\end{array}$ & 46.2748332 & -122.9145587 & $\begin{array}{l}\text { December 12, 1926- } \\
\quad \text { March 6, 2018 }\end{array}$ & $\begin{array}{l}\text { Daily mean streamflow, annual peak } \\
\text { streamflow, stage }\end{array}$ \\
\hline 48 & 14246000 & $\begin{array}{l}\text { Abernathy Creek Near Longview, } \\
\text { WA }\end{array}$ & 46.2026102 & -123.1553978 & $\begin{array}{l}\text { May 1, 1949- } \\
\text { December 31, } 1957\end{array}$ & $\begin{array}{l}\text { Daily mean streamflow, annual peak } \\
\text { streamflow }\end{array}$ \\
\hline 49 & 14246900 & $\begin{array}{l}\text { Columbia River at Port Westward, } \\
\text { Near Quincy, OR }{ }^{2}\end{array}$ & 46.18122136 & -123.1834539 & $\begin{array}{l}\text { May 1, 1968-March } \\
\quad 6,2018^{1}\end{array}$ & $\begin{array}{l}\text { Daily mean streamflow, annual peak } \\
\text { streamflow, stage }\end{array}$ \\
\hline 50 & 14247000 & $\begin{array}{l}\text { Clatskanie River Near Clatskanie, } \\
\text { OR }\end{array}$ & 46.048445 & -123.1234489 & $\begin{array}{l}\text { October } 1,1949- \\
\quad \text { October } 29,1954\end{array}$ & $\begin{array}{l}\text { Daily mean streamflow, annual peak } \\
\text { streamflow }\end{array}$ \\
\hline 51 & 14247500 & $\begin{array}{l}\text { Elochoman River Near Cathlamet, } \\
\text { WA }\end{array}$ & 46.2212209 & -123.3423492 & $\begin{array}{l}\text { October } 1,1940- \\
\text { October } 12,1971\end{array}$ & $\begin{array}{l}\text { Daily mean streamflow, annual peak } \\
\text { streamflow }\end{array}$ \\
\hline
\end{tabular}

${ }^{1}$ Station still active at the time of data compilation

${ }^{2}$ Station previously named Columbia River at Beaver Army Terminal, near Quincy, Oregon 
Table 5. Historical paper rating tables that were recreated within the internal U.S. Geological Survey database to expand and determine instantaneous discharge for flooding events.

[Rating number: Rating numbers were historically labeled as the date they were implemented, but the U.S. Geological Survey later switched to labeling them by numeral. Dates are in month-day-year. Abbreviations: blw, below; nr, near; OR, Oregon; WA, Washington]

\begin{tabular}{clcc}
\hline $\begin{array}{c}\text { Station } \\
\text { identification } \\
\text { number }\end{array}$ & Station name & Rating number & $\begin{array}{c}\text { Applied to } \\
\text { flooding event }\end{array}$ \\
\hline 14120000 & Hood River at Tucker Bridge, near Hood River, OR & 15 & 1986 \\
14137000 & Sandy River near Marmot, OR & $1-30-1935$ & 1933 \\
& & $1-31-1935$ & 1933 \\
14141500 & Little Sandy River near Bull Run, OR & 15 & 1974 \\
& & $2-27-1935$ & 1933 \\
14142500 & Sandy River blw Bull Run River, nr Bull Run, OR & $11-21-1933$ & 1974 \\
14191000 & Willamette River at Salem, OR & 14 & 1933 \\
& & 15 & 1974 \\
14200300 & Silver Creek at Silverton, OR & 17 & 1974 \\
& & 1 & 1986 \\
14202000 & Pudding River at Aurora, OR & 2 & 1965 \\
& & $3-15-1934$ & 1933 \\
14203500 & Tualatin River near Dilley, OR & $3-11-1935$ & 1933 \\
& & $3-3-1949$ & 1933 \\
14207500 & Tualatin River at West Linn, OR & 14 & 1974 \\
& & 15 & 1974 \\
& & $3-24-1934$ & 1933 \\
14210000 & Clackamas River at Estacada, OR & 7 & 1964 \\
14211500 & & 9 & 1974 \\
14243000 & Johnson Creek at Sycamore, OR & 10 & 1974 \\
\hline & Cowlitz River at Castle Rock, WA & 13 & 1974 \\
& & 14 & 1974 \\
& & 8 & 1974 \\
\hline
\end{tabular}

\section{Oregon Water Resources Department (OWRD)}

Twelve OWRD gages were compiled for this study (table 7; fig. 6). Data were downloaded for the stations of interest from the OWRD website (Oregon Water Resources Department, 2018a). Data prior to approximately 2016 could be downloaded from Historical Streamflow and Lake Level Data web application (Oregon Water Resources Department, 2018b), while more recent data could be found on the Near Real Time Hydrographics Data web application (Oregon Water Resources Department, 2018c). Daily-mean flows were available and retrieved from all 12 of the stations of interest. Annual peak flow files were available for 11 of the stations. All data were compiled in local standard time (PST). 
Table 6. Summary of U.S. Geological Survey historic flood data compiled in this report.

[Dates shown as month-day-year. Abbreviations: Ab, above; Bl, below, Cr, creek; Mt, Mount; N, North; Nr, near; OR, Oregon; Pc, Pine Creek; R, river; S, South; St, street; WA, Washington; × flood data available that year; -, no data available]

\begin{tabular}{|c|c|c|c|c|c|c|c|c|c|c|c|}
\hline \multirow{2}{*}{$\begin{array}{c}\text { Station } \\
\text { identifier }\end{array}$} & \multirow{2}{*}{ Station name } & \multirow{2}{*}{$\begin{array}{c}\text { Station } \\
\text { begin date }\end{array}$} & \multirow{2}{*}{$\begin{array}{l}\text { Station } \\
\text { end date }\end{array}$} & \multicolumn{8}{|c|}{ Flooding events of interest } \\
\hline & & & & 1933 & 1964 & 1974 & 1982 & 1986 & 1996 & 1997 & 2006 \\
\hline 14105700 & Columbia River at The Dalles, OR & $6-1-1878$ & $13-5-2018$ & - & - & - & - & - & $\mathrm{x}$ & - & $\mathrm{x}$ \\
\hline 14113000 & Klickitat River Near Pitt, WA & $7-1-1909$ & ${ }^{13}-5-2018$ & - & - & - & - & - & $\mathrm{x}$ & $\mathrm{x}$ & $\mathrm{x}$ \\
\hline 14113200 & Mosier Creek Near Mosier, OR & $4-17-1963$ & ${ }^{1} 3-5-2018$ & - & - & - & - & - & - & - & $\mathrm{x}$ \\
\hline 14120000 & Hood River at Tucker Bridge, Near Hood River, OR & $10-20-1897$ & ${ }^{13}-5-2018$ & - & $\mathrm{x}$ & - & $\mathrm{x}$ & $\mathrm{x}$ & $\mathrm{x}$ & $\mathrm{x}$ & $\mathrm{x}$ \\
\hline 14123500 & White Salmon River Near Underwood, WA & $11-1-1912$ & $13-5-2018$ & - & - & $\mathrm{x}$ & - & $\mathrm{x}$ & - & - & $\mathrm{x}$ \\
\hline 14125500 & Little White Salmon River Near Cook, WA & $10-1-1956$ & $10-5-1977$ & - & - & $\mathrm{x}$ & - & - & - & - & - \\
\hline 14137000 & Sandy River Near Marmot, OR & $9-1-1911$ & ${ }^{13}-5-2018$ & $\mathrm{x}$ & $\mathrm{x}$ & $\mathrm{x}$ & - & $\mathrm{x}$ & $\mathrm{x}$ & $\mathrm{x}$ & $\mathrm{x}$ \\
\hline 14141500 & Little Sandy River Near Bull Run, OR & $5-1-1911$ & ${ }^{1} 3-5-2018$ & $\mathrm{x}$ & $\mathrm{x}$ & $\mathrm{x}$ & - & $\mathrm{x}$ & $\mathrm{x}$ & $\mathrm{x}$ & $\mathrm{x}$ \\
\hline 14142500 & Sandy River Bl Bull Run River, Nr Bull Run, OR & $10-1-1910$ & ${ }^{13}-5-2018$ & $\mathrm{x}$ & $\mathrm{x}$ & - & - & $\mathrm{x}$ & $\mathrm{x}$ & $\mathrm{x}$ & $\mathrm{x}$ \\
\hline 14142800 & Beaver Creek at Troutdale, OR & 10-1-1999 & ${ }^{13}-5-2018$ & - & - & - & - & - & - & - & $\mathrm{x}$ \\
\hline 14191000 & Willamette River at Salem, OR & $10-1-1909$ & ${ }^{1} 3-5-2018$ & - & $\mathrm{x}$ & $\mathrm{x}$ & $\mathrm{x}$ & $\mathrm{x}$ & $\mathrm{x}$ & $\mathrm{x}$ & $\mathrm{x}$ \\
\hline 14194150 & South Yamhill River at Mcminnville, OR & $10-1-1994$ & $13-5-2018$ & - & - & - & - & - & $\mathrm{x}$ & $\mathrm{x}$ & $\mathrm{x}$ \\
\hline 14198400 & Bull Creek Near Wilhoit, OR & $3-31-1993$ & ${ }^{13}-5-2018$ & - & - & - & - & - & - & $\mathrm{x}$ & $\mathrm{x}$ \\
\hline 14198500 & Molalla R Ab Pc Nr Wilhoit, OR & $10-1-1935$ & $9-29-1993$ & - & $\mathrm{x}$ & $\mathrm{x}$ & - & $\mathrm{x}$ & - & - & - \\
\hline 14197000 & North Yamhill R at Pike, OR & $10-1-1948$ & $9-29-1973$ & - & $\mathrm{x}$ & - & - & - & - & - & - \\
\hline 14197900 & Willamette River at Newberg, OR & $10-1-2001$ & ${ }^{13}-5-2018$ & - & - & - & - & - & - & - & $\mathrm{x}$ \\
\hline 14198000 & Willamette River at Wilsonville, OR & $10-1-1948$ & $7-31-1973$ & - & $\mathrm{x}$ & - & - & - & - & - & - \\
\hline 14200000 & Molalla River Near Canby, OR & $8-1-1928$ & ${ }^{13}-5-2018$ & - & $\mathrm{x}$ & $\mathrm{x}$ & - & - & - & - & $\mathrm{x}$ \\
\hline 14200300 & Silver Creek at Silverton, OR & $10-1-1963$ & $2-5-2018$ & - & $\mathrm{x}$ & $\mathrm{x}$ & - & - & - & - & - \\
\hline 14201500 & Butte Creek at Monitor, OR & $1-1-1936$ & ${ }^{13}-5-2018$ & - & - & $\mathrm{x}$ & - & - & - & - & - \\
\hline 14201300 & Zollner Creek Near Mt Angel, OR & $7-1-1993$ & ${ }^{1} 3-6-2018$ & - & - & - & - & - & - & - & $\mathrm{x}$ \\
\hline 14201340 & Pudding River Near Woodburn, OR & $10-1-1997$ & ${ }^{13}-6-2018$ & - & - & - & - & - & - & - & $\mathrm{x}$ \\
\hline 14202000 & Pudding River at Aurora, OR & $10-1-1928$ & ${ }^{13}-6-2018$ & $\mathrm{x}$ & - & - & - & - & $\mathrm{x}$ & $\mathrm{x}$ & $\mathrm{x}$ \\
\hline 14203500 & Tualatin River Near Dilley, OR & $10-1-1939$ & $13-6-2018$ & - & $\mathrm{x}$ & $\mathrm{x}$ & - & $\mathrm{x}$ & $\mathrm{x}$ & $\mathrm{x}$ & $\mathrm{x}$ \\
\hline 14205400 & East Fork Dairy Creek Near Meacham Corner, OR & $5-8-2002$ & ${ }^{13}-6-2018$ & - & - & - & - & - & - & - & $\mathrm{x}$ \\
\hline 14206950 & Fanno Creek at Durham, OR & $10-1-1993$ & ${ }^{13}-6-2018$ & - & - & - & - & - & - & - & $\mathrm{x}$ \\
\hline 14207500 & Tualatin River at West Linn, OR & $8-1-1928$ & ${ }^{13}-6-2018$ & $\mathrm{x}$ & $\mathrm{x}$ & $\mathrm{x}$ & - & $\mathrm{x}$ & $\mathrm{x}$ & $\mathrm{x}$ & $\mathrm{x}$ \\
\hline 14211010 & Clackamas River Near Oregon City, OR & $6-1-2001$ & ${ }^{13}-6-2018$ & - & - & - & - & - & - & - & $\mathrm{x}$ \\
\hline 14210000 & Clackamas River at Estacada, OR & $4-1-1908$ & ${ }^{13}-6-2018$ & - & $\mathrm{x}$ & $\mathrm{x}$ & - & $\mathrm{x}$ & $\mathrm{x}$ & $\mathrm{x}$ & $\mathrm{x}$ \\
\hline 14211315 & Tryon Creek Near Lake Oswego, OR & $8-1-2001$ & ${ }^{13}-6-2018$ & - & - & - & - & - & - & - & $\mathrm{x}$ \\
\hline 14211500 & Johnson Creek at Sycamore, OR & $10-1-1940$ & ${ }^{13}-6-2018$ & - & $\mathrm{x}$ & $\mathrm{x}$ & - & $\mathrm{x}$ & $\mathrm{x}$ & $\mathrm{x}$ & $\mathrm{x}$ \\
\hline 14211550 & Johnson Creek at Milwaukie, OR & $4-22-1989$ & ${ }^{13}-6-2018$ & - & - & - & - & - & $\mathrm{x}$ & $\mathrm{x}$ & $\mathrm{x}$ \\
\hline
\end{tabular}




\begin{tabular}{|c|c|c|c|c|c|c|c|c|c|c|c|}
\hline \multirow{2}{*}{$\begin{array}{c}\text { Station } \\
\text { identifier }\end{array}$} & \multirow{2}{*}{ Station name } & \multirow{2}{*}{$\begin{array}{c}\text { Station } \\
\text { begin date }\end{array}$} & \multirow{2}{*}{$\begin{array}{l}\text { Station } \\
\text { end date }\end{array}$} & \multicolumn{8}{|c|}{ Flooding events of interest } \\
\hline & & & & 1933 & 1964 & 1974 & 1982 & 1986 & 1996 & 1997 & 2006 \\
\hline 14211720 & Willamette River at Portland, OR & $10-1-1972$ & ${ }^{13}-6-2018$ & - & - & - & - & - & - & - & $\mathrm{x}$ \\
\hline 14211814 & Fairview Creek at Glisan St Near Gresham, OR & $5-1-1992$ & ${ }^{13}-6-2018$ & - & - & - & - & - & $\mathrm{x}$ & $\mathrm{x}$ & $\mathrm{x}$ \\
\hline 14222500 & East Fork Lewis River Near Heisson, WA & $10-1-1929$ & ${ }^{13}-6-2018$ & - & - & - & - & - & $\mathrm{x}$ & - & $\mathrm{x}$ \\
\hline 14220500 & Lewis River at Ariel, WA & $7-1-1909$ & ${ }^{13}-6-2018$ & - & - & - & - & - & - & - & $\mathrm{x}$ \\
\hline 14243000 & Cowlitz River at Castle Rock, WA & $12-10-1926$ & ${ }^{13}-6-2018$ & - & - & $\mathrm{x}$ & - & - & $\mathrm{x}$ & $\mathrm{x}$ & $\mathrm{x}$ \\
\hline 14246900 & Columbia River at Port Westward, Near Quincy, OR & $5-1-1968$ & ${ }^{13}-6-2018$ & - & - & - & - & - & - & - & $\mathrm{x}$ \\
\hline 14192500 & S Yamhill R Nr Willamina, OR & $5-1-1934$ & 9-29-1993 & - & $\mathrm{x}$ & $\mathrm{x}$ & - & $\mathrm{x}$ & - & - & - \\
\hline 14193000 & Willamina $\mathrm{Cr}$ Nr Willamina, OR & $6-1-1934$ & 9-29-1991 & - & $\mathrm{x}$ & - & - & $\mathrm{x}$ & - & - & - \\
\hline 14194300 & N Yamhill R Nr Fairdale, OR & $10-1-1958$ & 9-29-1991 & - & $\mathrm{x}$ & $\mathrm{x}$ & - & $\mathrm{x}$ & - & - & - \\
\hline 14202500 & Tualatin R Nr Gaston, OR & $10-1-1940$ & $2-25-1985$ & - & - & - & $\mathrm{x}$ & - & - & - & - \\
\hline 14202920 & Sain Cr Nr Gaston, OR & $10-1-1972$ & $9-29-1976$ & - & - & $\mathrm{x}$ & - & - & - & - & - \\
\hline 14202850 & Scoggins Cr Ab Henry Hagg Lake Nr Gaston, OR & $10-1-1972$ & $9-29-1976$ & - & - & $\mathrm{x}$ & - & - & - & - & - \\
\hline 14202980 & Scoggins Cr Bl Henry Hagg Lake Nr Gaston, OR & $1-1-1975$ & $10-1-2006$ & - & - & - & - & $\mathrm{x}$ & $\mathrm{x}$ & $\mathrm{x}$ & $\mathrm{x}$ \\
\hline 14105700 & Columbia River at The Dalles, OR & $6-1-1878$ & ${ }^{13}-5-2018$ & - & - & - & - & - & $\mathrm{x}$ & - & $\mathrm{x}$ \\
\hline 14113000 & Klickitat River Near Pitt, WA & $7-1-1909$ & ${ }^{13}-5-2018$ & - & - & - & - & - & $\mathrm{x}$ & $\mathrm{x}$ & $\mathrm{x}$ \\
\hline 14113200 & Mosier Creek Near Mosier, OR & 4-17-1963 & ${ }^{13}-5-2018$ & - & - & - & - & - & - & - & $\mathrm{x}$ \\
\hline
\end{tabular}

${ }^{1}$ Station still active at the time of data compilation 
Table 7. Oregon Water Resources Department gaging station list for data compiled in this report.

[All stations were still active at the time of data compilation. Map no.: Map number. Station identifier: Names are presented with abbreviated terms as they are in the National Water Information System. Abbreviations: Ab, above; Bl, below; Cr, creek; Nr, near; OR, Oregon; R, river; Rd, road; rte, route; WA,

Washington]

\begin{tabular}{|c|c|c|c|c|c|c|}
\hline $\begin{array}{c}\text { Map } \\
\text { no. }\end{array}$ & $\begin{array}{c}\text { Station } \\
\text { identifier }\end{array}$ & Station name & Latitude & Longitude & Period of record & Data compiled \\
\hline 68 & 14192500 & $\begin{array}{l}\text { South Yamhill R Nr Willamina, } \\
\text { OR }\end{array}$ & $45^{\circ} 2^{\prime} 50.712^{\prime \prime}$ & $-123^{\circ} 30^{\prime} 15.451^{\prime \prime}$ & Sept. 30, 1993-Mar. 1, 2018 & $\begin{array}{l}\text { Daily mean streamflow, } \\
\text { annual peak streamflow }\end{array}$ \\
\hline 69 & 14193000 & Willamina $\mathrm{Cr} \mathrm{Nr}$ Willamina, OR & $45^{\circ} 8^{\prime} 35.671^{\prime \prime}$ & $-123^{\circ} 29^{\prime} 40.7^{\prime \prime}$ & Sept. 30, 1991-Mar. 1, 2018 & $\begin{array}{l}\text { Daily mean streamflow, } \\
\text { annual peak streamflow }\end{array}$ \\
\hline 70 & 14194300 & $\begin{array}{l}\text { North Yamhill R Nr Fairdale, } \\
\text { OR }\end{array}$ & $45^{\circ} 21^{\prime} 53.852^{\prime \prime}$ & $-123^{\circ} 22^{\prime} 45.48^{\prime \prime}$ & Sept. 30, 1991-Mar. 1, 2018 & $\begin{array}{l}\text { Daily mean streamflow, } \\
\text { annual peak streamflow }\end{array}$ \\
\hline 63 & 14202450 & $\begin{array}{l}\text { Tualatin R Bl Lee Falls Nr } \\
\text { Cherry Grove, OR }\end{array}$ & $45^{\circ} 27^{\prime} 37.699^{\prime \prime}$ & $-123^{\circ} 16^{\prime} 45.419^{\prime \prime}$ & Jan. 2, 2008-Mar. 1, 2018 & $\begin{array}{l}\text { Daily mean streamflow, } \\
\text { annual peak streamflow }\end{array}$ \\
\hline 71 & 14202850 & $\begin{array}{l}\text { Scoggins Cr Ab Henry Hagg } \\
\text { Lake Nr Gaston, OR }\end{array}$ & $45^{\circ} 29^{\prime} 59.791^{\prime \prime}$ & $-123^{\circ} 15^{\prime} 4.277^{\prime \prime}$ & Sept. 30, 1976-Mar. 1, 2018 & $\begin{array}{l}\text { Daily mean streamflow, } \\
\text { annual peak streamflow }\end{array}$ \\
\hline 72 & 14202920 & Sain Cr Nr Gaston, OR & $45^{\circ} 28^{\prime} 50.891^{\prime \prime}$ & $-123^{\circ} 14^{\prime} 45.19^{\prime \prime}$ & Sept. 30, 1976-Mar. 1, 2018 & $\begin{array}{l}\text { Daily mean streamflow, } \\
\text { annual peak streamflow }\end{array}$ \\
\hline 73 & 14202980 & $\begin{array}{l}\text { Scoggins Cr Bl Henry Hagg } \\
\text { Lake Nr Gaston, OR }\end{array}$ & $45^{\circ} 28^{\prime} 18.322^{\prime \prime}$ & $-123^{\circ} 11^{\prime} 50.881^{\prime \prime}$ & Oct. 2, 2006-Mar. 1, 2018 & $\begin{array}{l}\text { Daily mean streamflow, } \\
\text { annual peak streamflow }\end{array}$ \\
\hline 64 & 14204530 & $\begin{array}{l}\text { Gales Cr At Route } 47 \text { At Forest } \\
\text { Grove, OR }\end{array}$ & $45^{\circ} 30^{\prime} 38.801^{\prime \prime}$ & $-123^{\circ} 6^{\prime} 55.829^{\prime \prime}$ & Jan. 1, 2008-Mar. 1, 2018 & $\begin{array}{l}\text { Daily mean streamflow, } \\
\text { annual peak streamflow }\end{array}$ \\
\hline 65 & 14204800 & $\begin{array}{l}\text { Tualatin R at Golf Course Rd Nr } \\
\text { Cornelius, OR }\end{array}$ & $45^{\circ} 30^{\prime} 7.549^{\prime \prime}$ & $-123^{\circ} 3^{\prime} 22.061^{\prime \prime}$ & Jan. 1, 2008-Jan. 25, 2018 & $\begin{array}{l}\text { Daily mean streamflow, } \\
\text { annual peak streamflow }\end{array}$ \\
\hline 66 & 14206200 & $\begin{array}{l}\text { Dairy Cr At Rte } 8 \text { Nr Hillsboro, } \\
\text { OR }\end{array}$ & $45^{\circ} 31^{\prime} 12.41^{\prime \prime}$ & $-123^{\circ} 0^{\prime} 41.08^{\prime \prime}$ & Feb. 23, 2008-Jan. 1, 2018 & Daily mean streamflow \\
\hline 67 & 14206295 & $\begin{array}{l}\text { Tualatin R at Rood Bridge At } \\
\text { Hillsboro, OR }\end{array}$ & $45^{\circ} 29^{\prime} 23.86^{\prime \prime}$ & $-122^{\circ} 57^{\prime} 5.558^{\prime \prime}$ & Jan. 1, 2008-Jan. 25, 2018 & $\begin{array}{l}\text { Daily mean streamflow, } \\
\text { annual peak streamflow }\end{array}$ \\
\hline 74 & 14206500 & Tualatin $\mathrm{R}$ at Farmington, OR & $45^{\circ} 26^{\prime} 57.71^{\prime \prime}$ & $-122^{\circ} 57^{\prime} 2.588^{\prime \prime}$ & Sept. 30, 1976-Jan. 25, 2018 & $\begin{array}{l}\text { Daily mean streamflow, } \\
\text { annual peak streamflow }\end{array}$ \\
\hline
\end{tabular}




\section{National Oceanic and Atmospheric Administration (NOAA)}

Of the 12 long-term NOAA tidal gages compiled for this study, 6 were coastal locations in Washington, Oregon, and California, and 6 were river locations in the Columbia and Willamette Rivers (table 8; fig. 2). Data were downloaded for each station from the NOAA tides and currents webpage (National Oceanic and Atmospheric Administration, 2018a). All data were compiled in local standard time (PST) and in station datum (STND), an arbitrary fixed base elevation.

Table 8. National Oceanic and Atmospheric Administration gaging station list for data compiled in this report.

[All stations were still active at time of data compilation. Map no.: Map number. Period of record may not be continuous. Abbreviations: CA, California; OR, Oregon; St, street; WA, Washington]

\begin{tabular}{cclclll}
\hline $\begin{array}{c}\text { Map } \\
\text { no. }\end{array}$ & $\begin{array}{c}\text { Station } \\
\text { identifier }\end{array}$ & \multicolumn{1}{c}{ Station name } & Latitude & Longitude & Period of record & Data compiled \\
\hline \multicolumn{7}{c}{ Coastal locations } \\
\hline 75 & 9443090 & Neah Bay, WA & $48^{\circ} 22.2^{\prime} \mathrm{N}$ & $124^{\circ} 36.1^{\prime} \mathrm{W}$ & July 1934-Dec. 2017 & Hourly elevation \\
76 & 9440910 & Toke Point, WA & $46^{\circ} 42.5^{\prime} \mathrm{N}$ & $123^{\circ} 58^{\prime} \mathrm{W}$ & Nov. 1972-Dec. 2017 & Hourly elevation \\
86 & 9439040 & Astoria, OR & $46^{\circ} 124^{\prime} \mathrm{N}$ & $123^{\circ} 46.1^{\prime} \mathrm{W}$ & Jan. 1925-Dec. 2017 & Hourly elevation \\
77 & 9435380 & South Beach, OR & $44^{\circ} 37.5^{\prime} \mathrm{N}$ & $124^{\circ} 327^{\prime} \mathrm{W}$ & Jan. 1967-Dec. 2017 & Hourly elevation \\
78 & 9432780 & Charleston, OR & $43^{\circ} 20.7^{\prime} \mathrm{N}$ & $124^{\circ} 19.3^{\prime} \mathrm{W}$ & Mar. 1970-Dec. 2017 & Hourly elevation \\
79 & 9419750 & Crescent City, CA & $41^{\circ} 44.7^{\prime} \mathrm{N}$ & $124^{\circ} 11.1^{\prime} \mathrm{W}$ & Apr. 1933-Dec. 2017 & Hourly elevation \\
\hline \multicolumn{7}{c}{ River locations } \\
\hline 80 & 9440569 & Skamokawa, WA & $46^{\circ} 16.2^{\prime} \mathrm{N}$ & $123^{\circ} 27.4^{\prime} \mathrm{W}$ & Mar. 2002-Dec. 2017 & Hourly elevation \\
82 & 9440422 & Longview, WA & $46^{\circ} 6.4^{\prime} \mathrm{N}$ & $122^{\circ} 57.2^{\prime} \mathrm{W}$ & Mar. 2002-Dec. 2017 & Hourly elevation \\
83 & 9439201 & St. Helens, OR & $45^{\circ} 51.8^{\prime} \mathrm{N}$ & $122^{\circ} 47.8^{\prime} \mathrm{W}$ & Mar. 2002-Dec. 2017 & Hourly elevation \\
84 & 9440083 & Vancouver, WA & $45^{\circ} 37.9^{\prime} \mathrm{N}$ & $122^{\circ} 41.8^{\prime} \mathrm{W}$ & Mar. 2002-Dec. 2017 & Hourly elevation \\
85 & 9439099 & Wauna, OR & $46^{\circ} 9.7^{\prime} \mathrm{N}$ & $123^{\circ} 24.5^{\prime} \mathrm{W}$ & Mar. 2002-Dec. 2017 & Hourly elevation \\
81 & 9439221 & Portland Morrison & $45^{\circ} 30.6^{\prime} \mathrm{N}$ & $122^{\circ} 40.4^{\prime} \mathrm{W}$ & Mar. 2002-Jan. 2009 & Hourly Elevation \\
& \multicolumn{7}{c}{ St Bridge, OR } & & & \\
\hline
\end{tabular}

Washington Department of Ecology (WA-DOE)

Thirteen WA-DOE gages were compiled for this study (table 9; figs. 3, 4, 5, and 8). Data were downloaded from each available year of data from the WA-DOE Flow Monitoring Network webpage (Department of Ecology State of Washington, 2018). Daily-mean streamflow values were retrieved from 12 of the gages, and 15-minute stage data were retrieved from 6 of the gages. All gages were established after January 2000; therefore, only the January 2006 flooding event was available and retrieved from six of the gages. All data were compiled in local standard time (PST) and all stage data were compiled at an arbitrary datum.

\section{Pacific Northwest National Laboratory (PNNL)}

Thirty-nine PNNL elevation gages were compiled for this study (table 10; figs. 3, 4, 5, and 8). Data were supplied by the U.S. Army Corps of Engineers (USACE) in a Microsoft Excel data file (R. Cahill, U.S. Army Corps of Engineers, written commun., April 26, 2018). Elevation data were available in either 30-minute or hourly increments. The original datasets were supplied in Greenwich Mean Time (GMT) with a gage datum of NAVD 88 in meters. 
Table 9. Washington Department of Ecology gaging station list for data compiled in this report.

[Map no.: Map number. Period of record: Station still active at the time of data compilation; may not be continuous. Abbreviations: blw, below; Cr, creek; Dr, Drive; EF, East Fork; nr, near; R, river; Rd, road; St, street]

\begin{tabular}{|c|c|c|c|c|c|c|}
\hline $\begin{array}{l}\text { Map } \\
\text { no. }\end{array}$ & $\begin{array}{c}\text { Station } \\
\text { identifier }\end{array}$ & Station name & Latitude & Longitude & Period of record & Data compiled \\
\hline 87 & $30 \mathrm{C} 070$ & $\begin{array}{l}\text { Little Klickitat R nr } \\
\text { Wahkiacus }\end{array}$ & $45^{\circ} 50^{\prime} 35.00061^{\prime \prime}$ & $-121^{\circ} 3^{\prime} 28.98743^{\prime \prime}$ & June 2004-Sept. $2017^{1}$ & $\begin{array}{l}\text { Daily mean streamflow, } 2006 \text { flood } \\
\text { event } 15 \text {-min streamflow }\end{array}$ \\
\hline 88 & $29 \mathrm{C} 100$ & Wind $\mathrm{R}$ at Stabler & $45^{\circ} 48^{\prime} 27.36^{\prime \prime}$ & $-121^{\circ} 54^{\prime} 32.04^{\prime \prime}$ & June 2004-Sept. $2017^{1}$ & Daily mean streamflow \\
\hline 89 & $29 \mathrm{~A} 070$ & Rock $\mathrm{Cr}$ at Stevenson & $45^{\circ} 41^{\prime} 55.31982^{\prime \prime}$ & $-121^{\circ} 54^{\prime} 17.28149^{\prime \prime}$ & June 2008-Sept 2013 & Daily mean streamflow \\
\hline 90 & 28B080 & $\begin{array}{l}\text { Washougal R at Hathaway } \\
\text { Park }\end{array}$ & $45^{\circ} 35^{\prime} 2.04^{\prime \prime}$ & $-122^{\circ} 20^{\prime} 35.88^{\prime \prime}$ & Jan. 2005-Sept. $2017^{1}$ & $\begin{array}{l}\text { Daily mean streamflow, } 15-\mathrm{min} \\
\text { stage, } 2006 \text { flood event } 15-\mathrm{min} \\
\text { Streamflow }\end{array}$ \\
\hline 91 & 27D090 & $\begin{array}{l}\text { EF Lewis R nr Dollar } \\
\text { Corner }\end{array}$ & $45^{\circ} 48^{\prime} 51.12^{\prime \prime}$ & $-122^{\circ} 35^{\prime} 30.12^{\prime \prime}$ & Jan. 2005-Sept 2013 & $\begin{array}{l}\text { Daily mean streamflow, } 2006 \text { flood } \\
\text { event } 15 \text {-min Streamflow }\end{array}$ \\
\hline 92 & $26 \mathrm{C} 075$ & Coweeman R nr Kelso & $46^{\circ} 7^{\prime} 41.16486^{\prime \prime}$ & $-122^{\circ} 50^{\prime} 17.8894 "$ & $\begin{array}{l}\text { Sept. 2006-Sept. } \\
2017^{1}\end{array}$ & $\begin{array}{l}\text { Daily mean streamflow, } 15-\mathrm{min} \\
\text { Stage }\end{array}$ \\
\hline 93 & $25 \mathrm{~F} 060$ & Mill Cr at Mouth & $46^{\circ} 11^{\prime} 26.16486^{\prime \prime}$ & $-123^{\circ} 10^{\prime} 42.94739^{\prime \prime}$ & June 2004-Sept. $2017^{1}$ & $\begin{array}{l}\text { Daily mean streamflow, } 15-\mathrm{min} \\
\text { stage, } 2006 \text { flood event } 15-\mathrm{min} \\
\text { streamflow }\end{array}$ \\
\hline 94 & $25 \mathrm{E} 060$ & Abernathy Cr nr Mouth ${ }^{2}$ & $46^{\circ} 11^{\prime} 43.0838^{\prime \prime}$ & $-123^{\circ} 9^{\prime} 56.1731^{\prime \prime}$ & June 2004-Sept. $2017^{1}$ & Daily mean streamflow, 15 -min stage \\
\hline 95 & $25 \mathrm{D} 050$ & Germany $\mathrm{Cr}$ at Mouth & $46^{\circ} 11^{\prime} 29.03503^{\prime \prime}$ & $-123^{\circ} 7^{\prime} 30^{\prime \prime}$ & June 2004-Sept. $2017^{1}$ & $\begin{array}{l}\text { Daily mean streamflow, } 15-\mathrm{min} \\
\text { stage, } 2006 \text { flood event } 15-\mathrm{min} \\
\text { Streamflow }\end{array}$ \\
\hline 96 & $25 \mathrm{C} 060$ & Elochoman R at Monroe Dr & $46^{\circ} 13^{\prime} 32.16431^{\prime \prime}$ & $-123^{\circ} 21^{\prime} 32.40417^{\prime \prime}$ & Dec. $2014-$ Sept. $2017^{1}$ & Daily mean streamflow \\
\hline 97 & $25 \mathrm{~B} 060$ & Grays R nr Mouth & $46^{\circ} 21^{\prime} 15.11444^{\prime \prime}$ & $-123^{\circ} 34^{\prime} 53.03284^{\prime \prime}$ & Jan. 2005-Sept. $2013^{2}$ & $\begin{array}{l}\text { Daily mean streamflow, } 2006 \text { flood } \\
\text { event } 15 \text {-min Streamflow }\end{array}$ \\
\hline 98 & $24 \mathrm{~N} 100$ & Chinook R nr Houchen St & $46^{\circ} 16^{\prime} 4.8^{\prime \prime}$ & $-123^{\circ} 55^{\prime} 24.96^{\prime \prime}$ & Dec. 2014-Sept. $2017^{1}$ & Daily mean streamflow \\
\hline 99 & 24N070 & $\begin{array}{l}\text { Chinook R at Chinook } \\
\text { Valley Rd }\end{array}$ & $46^{\circ} 17^{\prime} 0.6^{\prime \prime}$ & $-123^{\circ} 56^{\prime} 15^{\prime \prime}$ & Dec. $2014-$ Sept. $2017^{1}$ & 15-min stage \\
\hline
\end{tabular}

${ }^{1}$ Website states station still active but no additional data is available online after Sept. 2013 at the time of data compilation.

${ }^{2}$ Station was moved to another location on same creek starting June 28, 2006. 
Table 10. Pacific Northwest National Laboratory gaging station list for data compiled in this report.

[Map no.: Map number. Period of record may not be continuous]

\begin{tabular}{|c|c|c|c|c|c|c|}
\hline $\begin{array}{c}\text { Map } \\
\text { no. }\end{array}$ & $\begin{array}{l}\text { Station } \\
\text { identifier }\end{array}$ & Station name & $\begin{array}{l}\text { Latitude } \\
\text { (decimal } \\
\text { degrees) }\end{array}$ & $\begin{array}{c}\text { Longitude } \\
\text { (decimal } \\
\text { degrees) }\end{array}$ & Period of record & Data compiled \\
\hline 100 & BBM-HOBO & Baker Bay Marsh hobo & 46.300657 & -124.04549 & Apr. 2011-Feb. 2017 & Hourly elevation \\
\hline 101 & BIM-HOBO & Burke Island Marsh Hobo sensor & 45.939161 & -122.790358 & July 2011-May 2012 & Hourly elevation \\
\hline 102 & BSM-HOBO & Bradwood Slough Marsh Hobo sensor & 46.203008 & -123.445919 & July 2009-Aug. 2010 & Hourly elevation \\
\hline 103 & CCR-HOBO & Coal Creek Riparian Wetland Hobo sensor & 46.167735 & -123.038533 & July 2008-Aug. 2009 & Hourly elevation \\
\hline 104 & CCS-HOBO & Crooked Creek Swamp hobo & 46.294423 & -123.672489 & Aug. 2007-June 2010 & 30-min elevation \\
\hline 105 & CHM-HOBO & Chinook River Marsh hobo & 46.298528 & -123.96906 & Aug. 2008-Aug. 2009 & Hourly elevation \\
\hline 106 & CI1-HOBO & Cottonwood Island 1 hobo & 46.080391 & -122.876356 & Mar. 2009-Aug. 2010 & Hourly elevation \\
\hline 107 & CLM-HOBO & Cunningham Lake Marsh Hobo sensor & 45.807547 & -122.805071 & July 2009-Feb. 2017 & Hourly elevation \\
\hline 108 & CRM-HOBO & Clatskanie River Marsh Hobo sensor & 46.13337 & -123.233933 & Aug. 2008-July 2009 & Hourly elevation \\
\hline 109 & CS1-HOBO & Campbell Slough Marsh Hobo sensor & 45.784739 & -122.753966 & July 2008-Feb. 2017 & Hourly elevation \\
\hline 110 & DSC-HOBO & Dibblee Slough Marsh hobo & 46.114992 & -122.995492 & Mar. 2009-Aug. 2010 & Hourly elevation \\
\hline 111 & FCB-HOBO & Ft. Clatsop Dike-breach Marsh hobo & 46.131636 & -123.878962 & Aug. 2008-June 2009 & 30-min elevation \\
\hline 112 & FLM-HOBO & Franz Lake Marsh Hobo sensor & 45.60062 & -122.102936 & July 2008-July 2017 & Hourly elevation \\
\hline 113 & GCR-HOBO & Gee Creek Riparian Wetland Hobo sensor & 45.847913 & -122.778405 & Aug. 2009-July 2010 & Hourly elevation \\
\hline 114 & GIC-HOBO & Goat Island Marsh Hobo sensor & 45.932088 & -122.815116 & Aug. 2008-Aug. 2012 & Hourly elevation \\
\hline 115 & GIM-HOBO & Grant Island Marsh Hobo sensor & 46.123443 & -123.804429 & June 2009-June 2010 & Hourly elevation \\
\hline 116 & GOM-HOBO & Government Island Marsh Hobo sensor & 45.591347 & -122.561208 & Nov. 2011-Nov. 2012 & Hourly elevation \\
\hline 117 & HCM-HOBO & Hardy Creek Marsh Hobo sensor & 45.629163 & -122.006155 & July 2008-July 2009 & Hourly elevation \\
\hline 118 & HIB-HOBO & Haven Island Dike-breach Marsh Hobo sensor & 46.117643 & -123.81049 & June 2009-June 2010 & Hourly elevation \\
\hline 119 & KIB-HOBO & Karlson Island Dike-breach Marsh hobo & 46.203357 & -123.613757 & Aug. 2007-July 2008 & 30-min elevation \\
\hline 120 & KIS-HOBO & Karlson Island Swamp hobo & 46.188787 & -123.600257 & Aug. 2007-July 2008 & 30-min elevation \\
\hline 121 & LI2-HOBO & Lord Island Marsh 2 Hobo sensor & 46.135579 & -123.029157 & Aug. 2008-July 2009 & Hourly elevation \\
\hline 122 & LRI-HOBO & Little Ryan Island Hobo Sensor & 46.197455 & -123.403081 & April 2014-Feb. 2015 & Hourly elevation \\
\hline 123 & OWM-HOBO & Washougal River (Old) Marsh Hobo sensor & 45.580987 & -122.394679 & Nov. 2011-Nov. 2012 & Hourly elevation \\
\hline 124 & PIM-HOBO & Pierce Island Marsh Hobo sensor & 45.620655 & -122.010976 & July 2008-July 2009 & Hourly elevation \\
\hline 125 & RI2-HOBO & Reed Island Slough Marsh Hobo sensor & 45.555386 & -122.298962 & Nov. 2001-Oct 2012 & Hourly elevation \\
\hline 126 & RIM-HOBO & Ryan Island Marsh Hobo sensor & 46.207838 & -123.414729 & July 2009-Aug. 2010 & Hourly elevation \\
\hline 127 & SIM-HOBO & Sand Island (Rooster Rock) Marsh Hobo sensor & 45.553465 & -122.212189 & July 2008-July 2009 & Hourly elevation \\
\hline 128 & SRM-HOBO & Secret River Marsh Hobo sensor & 46.304789 & -123.694938 & July 2007-Aug. 2016 & Hourly elevation \\
\hline 129 & SSC-HOBO & Sauvie Willow Bar Marsh Hobo sensor & 45.736777 & -122.77199 & July 2009-July 2010 & Hourly elevation \\
\hline 130 & SSS-HOBO2 & Seal Slough Swamp Hobo sensor 2 & 46.326466 & -123.661548 & Feb. 2010-June 2010 & 30-min elevation \\
\hline 131 & WAC-HOBO & Wallace Island West Marsh Hobo sensor & 46.140476 & -123.282894 & July 2009-July 2010 & Hourly elevation \\
\hline 132 & WHC-HOBO & Whites Island Marsh Hobo sensor & 46.159321 & -123.338159 & July 2009-Feb. 2017 & Hourly elevation \\
\hline
\end{tabular}




\begin{tabular}{|c|c|c|c|c|c|c|}
\hline $\begin{array}{c}\text { Map } \\
\text { no. }\end{array}$ & $\begin{array}{l}\text { Station } \\
\text { identifier }\end{array}$ & Station name & $\begin{array}{l}\text { Latitude } \\
\text { (decimal } \\
\text { degrees) }\end{array}$ & $\begin{array}{c}\text { Longitude } \\
\text { (decimal } \\
\text { degrees) }\end{array}$ & Period of record & Data compiled \\
\hline 133 & WI2-HOBO & Welch Island Marsh hobo & 46.255479 & -123.484440 & Dec. 2011-Feb. 2017 & Hourly elevation \\
\hline 134 & WIM-HOBO & Welch Island Marsh Slough hobo & 46.253 & -123.481181 & July 2008-Aug. 2009 & Hourly elevation \\
\hline 135 & WR2-HOBO & Walluski River Marsh Hobo sensor & 46.133571 & -123.782039 & June 2008-June 2009 & Hourly elevation \\
\hline 136 & WRB-HOBO & Walluski River Dike-breach Marsh Hobo sensor & 46.133577 & -123.782054 & June 2008-June 2009 & Hourly elevation \\
\hline 137 & WRM-HOBO & Walluski River Mouth Marsh Hobo sensor ${ }^{1}$ & 46.14398 & -123.789197 & July 2009-July 2010 & Hourly elevation \\
\hline 138 & WSS-HOBO & Westport Slough Shrub Hobo sensor & 46.121457 & -123.345692 & Aug. 2009-July 2010 & Hourly elevation \\
\hline
\end{tabular}

${ }^{1}$ Site named "Washogal River Mouth Marsh Hobo sensor" in original data file. Adjusted to "Walluski River Mouth Marsh Hobo sensor" based on latitude/longitude coordinates.

Table 11. Portland State University gaging station list for data compiled in this report.

[Map no.: Map number. Period of record may not be continuous. OR, Oregon;--, stations not given identifier numbers]

\begin{tabular}{rcllll}
\hline Map no. & Station identifier & \multicolumn{1}{c}{ Station name } & \multicolumn{1}{c}{ Latitude } & \multicolumn{1}{c}{ Longitude } & \multicolumn{1}{c}{ Period of record } \\
\hline 141 & - & Astoria & $46^{\circ} 11^{\prime} 26.16^{\prime \prime} \mathrm{N}$ & $123^{\circ} 49^{\prime} 48.5682^{\prime \prime} \mathrm{W}$ & Jan. 1855-Oct. 1876 \\
139 & 9439026 & Astoria Youngs Bay, OR & $46^{\circ} 10.3^{\prime} \mathrm{N}$ & $123^{\circ} 50.5^{\prime} \mathrm{W}$ & Elevation \\
86 & 9439040 & Astoria Tongue Point, OR & $46^{\circ} 12.4^{\prime} \mathrm{N}$ & $123^{\circ} 46.1^{\prime} \mathrm{W}$ & Mar. 1931-Feb. 1943 \\
140 & - & Portland, OR & $45^{\circ} 31^{\prime} 3.579^{\prime \prime} \mathrm{N}$ & $122^{\circ} 40^{\prime} 13.296^{\prime \prime} \mathrm{W}$ & Elevation \\
\hline
\end{tabular}

${ }^{1}$ Station still active at the time of data compilation 


\section{Portland State University (PSU)}

Data were provided by PSU (Dr. Stefan Talke, Portland State University, written commun., September 24, 2018) from a recently published paper (Talke and Jay, 2017). Data from four elevation gaging stations were compiled for this study (table 11; figs. 2, 3, and 7). Data directly retrieved from the NOAA webpage were used to populate the Astoria Tongue Point, OR station (9439026). The corrected data file for 9439026 includes a stage datum correction applied to documented instability of BM1 noted in Burgette and others (2009). The data were supplied in Greenwich Mean Time (GMT) with a gage datum of NAVD 88 in meters.

\section{United States Army Corps of Engineers (USACE) Dataquery}

Data (table 12; figs. 2, 4, 5, 7, and 8) to supplement and (or) extend data records at five sites were provided by the USACE in the form of HEC-DSS files that were retrieved from the Dataquery/Corps Water Management System (U.S. Army Corps of Engineers, 2018b). Table 13 details a selection of digitized paper files from post-flood report data compiled by USACE that were also included as part of this data compilation (U.S. Army Corps of Engineers, 1943, 1949, 1956, 1957, 1963, 1966, and 1975; Columbia Basin Inter-Agency Committee, 1966; Northwest Hydraulic Consultants and others, 1997). Seasonal peak stages (winter and spring) for Willamette at Portland (PRTO) and Columbia at Vancouver (VANW) were digitized from USACE drawing CL-03-112 and CL-03-116. USACE also operates a network of stage gages on the Lower Cowlitz River since the mid-2000s, these were not included in this analysis because they are located on the tributary and not the main stem along with the shorter period of record. High-water marks along the Columbia for the 1948, 1956, and 1996 floods exist in USACE records, but are not included here because they representative of the whole river and are not tied to a specific gage. Additional daily data from Dataquery containing maximum, mean, and minimum stage at VANW are also included (1974-2003), though other datasets should take precedence during periods of overlap due to uncertainty about the source of these data. 
Table 12. U.S. Army Corps of Engineers gaging station list for data compiled in this report.

[Period of record: may not be continuous.]

\begin{tabular}{|c|c|c|c|c|c|c|}
\hline $\begin{array}{c}\text { Map } \\
\text { no. }\end{array}$ & $\begin{array}{c}\text { Station } \\
\text { identifier }\end{array}$ & Station name & Latitude & Longitude & Period of record & Data Compiled \\
\hline 62 & $\mathrm{BON}$ & Bonneville Lock and Dam & $45^{\circ} 37^{\prime} 59^{\prime \prime} \mathrm{N}$ & $121^{\circ} 57^{\prime} 39^{\prime \prime} \mathrm{W}$ & Sept. 30, 1960-Oct. 2, $2018^{1}$ & Tailwater elevation, Streamflow \\
\hline 59 & BON-PH1 & Bonneville Powerhouse 1 & $45^{\circ} 38^{\prime} 22^{\prime \prime} \mathrm{N}$ & $121^{\circ} 56^{\prime} 51^{\prime \prime} \mathrm{W}$ & Nov. 29, 1966-Oct. 2, $2018^{1}$ & Tailwater elevation \\
\hline 60 & TDA & The Dalles & $45^{\circ} 36^{\prime} 48^{\prime \prime} \mathrm{N}$ & $121^{\circ} 8^{\prime} 4^{\prime \prime} \mathrm{W}$ & Nov. 29, 1966-Aug. 2, $2018^{1}$ & Streamflow \\
\hline 53 & $\mathrm{COCO}$ & Columbia at Columbia City & $45^{\circ} 54^{\prime} 0^{\prime \prime} \mathrm{N}$ & $122^{\circ} 48^{\prime} 0^{\prime \prime} \mathrm{W}$ & Oct. 1, 1974-Mar. 30, 1982 & Elevation \\
\hline 61 & CAMW & Columbia River at Camas & $45^{\circ} 34^{\prime} 48^{\prime \prime} \mathrm{N}$ & $122^{\circ} 25^{\prime} 12^{\prime \prime} \mathrm{W}$ & Oct. 2, 1974-May 19, 1987 & Stage \\
\hline
\end{tabular}

Table 13. U.S. Army Corps of Engineers post-flood report data compiled for this report.

[-, stations not given identifier numbers]

\begin{tabular}{|c|c|c|c|c|}
\hline $\begin{array}{c}\text { Station } \\
\text { identifier }\end{array}$ & Station name & Latitude & Longitude & Post flood report data compiled \\
\hline- & Clackamas at Clackamas & $45^{\circ} 23^{\prime} 50^{\prime \prime} \mathrm{N}$ & $122^{\circ} 33^{\prime} 27^{\prime \prime} \mathrm{W}$ & 1974 streamflow \\
\hline - & Columbia at Fort Stevens & Unknown & Unknown & 1956 stage \\
\hline LONW & Columbia at Longview & $46^{\circ} 6.4^{\prime} \mathrm{N}$ & $122^{\circ} 57.2^{\prime} \mathrm{W}$ & $\begin{array}{l}1948 \text { daily max \& daily min stage, } 1956 \text { stage, } 1964 \text { peak stage, } 1974 \\
\text { peak stage }\end{array}$ \\
\hline SHNO & Columbia at St Helens & $45^{\circ} 51.8^{\prime} \mathrm{N}$ & $122^{\circ} 47.8^{\prime} \mathrm{W}$ & 1956 stage, 1964 peak stage \\
\hline VANW & Columbia at Vancouver & $45^{\circ} 37^{\prime} 13.409^{\prime \prime} \mathrm{N}$ & $122^{\circ} 40^{\prime} 28.346^{\prime \prime} \mathrm{W}$ & $\begin{array}{l}1943 \text { peak stage, } 1948 \text { daily max\& daily min stage, } 1956 \text { stage, } 1965 \\
\text { stage, } 1974 \text { stage }\end{array}$ \\
\hline 14202000 & Pudding at Aurora & $45^{\circ} 14^{\prime} 0.437^{\prime \prime} \mathrm{N}$ & $122^{\circ} 45^{\prime} 0.326^{\prime \prime} \mathrm{W}$ & 1964 streamflow \\
\hline 14194000 & $\begin{array}{l}\text { South Yamhill near } \\
\text { Whiteson }\end{array}$ & $45^{\circ} 10^{\prime} 08^{\prime \prime} \mathrm{N}$ & $123^{\circ} 12^{\prime} 25^{\prime \prime} \mathrm{W}$ & 1964 streamflow, 1974 streamflow, 1996 streamflow \\
\hline ORC & Willamette at Oregon City & $45^{\circ} 20^{\prime} 53.428^{\prime \prime} \mathrm{N}$ & $122^{\circ} 37^{\prime} 16.341^{\prime \prime} \mathrm{W}$ & 1943 stage, 1955 flow, 1961 stage, 1964 flow, 1974 flow \\
\hline PRTO & Willamette at Portland & $45^{\circ} 31^{\prime} 5.426^{\prime \prime} \mathrm{N}$ & $122^{\circ} 40^{\prime} 8.346^{\prime \prime} \mathrm{W}$ & 1943 peak stage, 1948 stage, 1956 stage, 1964 flow, 1974 stage \\
\hline
\end{tabular}




\section{Review and Quality Assurance/Quality Control (QA/QC) of Acquired Datasets}

\section{General Data Review}

HEC-DSS software was used to review data for accuracy, and any points deemed erroneous, based on hydrologic judgement, were removed (fig. 9). Nearby gages, if available, were also used for comparison to help evaluate areas of uncertainty where the data appeared to deviate from the general trend. If erroneous data were removed from a dataset, the source file was duplicated to maintain an unaltered file. Both the original and final datasets were published as HEC-DSS database files (Boudreau and others, 2021). The time datum of all data compiled were reviewed and adjusted when needed to get all datasets consistently in the PST time datum. The gage elevation datums were also reviewed and adjusted when possible to get all datasets into a consistent datum (NAVD 88).
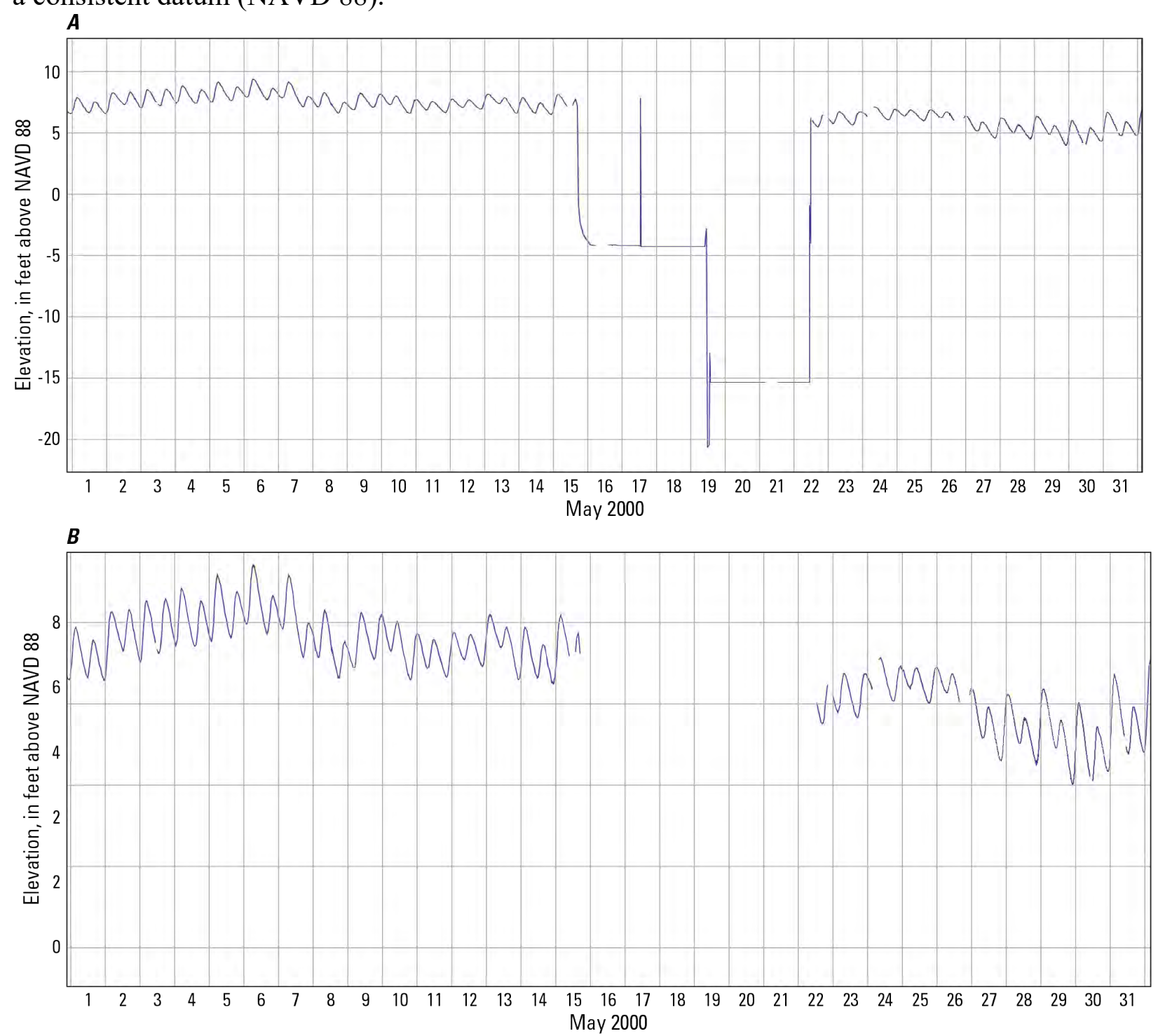

Figure 9. Raw elevation data that included an example of erroneous data $(A)$ and the edited dataset with the erroneous data removed (B), St. Helens, Oregon. May 2000. NAVD 88, North American Vertical Datum of 1988 . 


\section{Time Datum Adjustments}

For this study, most data were collected in or shifted to Pacific Standard Time (PST). The data from both PNNL and PSU were obtained in GMT and shifted accordingly, including date adjustments where needed. The USACE Dataquery files were supplied in local time, both PST and Pacific Daylight Time (PDT). USACE data in PDT were not shifted because the one-hour time difference was surmised to have negligible impact on the calculated summary maximums.

\section{Vertical Gage Datum Adjustments}

Most data were collected in or adjusted to North American Vertical Datum of 1988 (NAVD 88) in feet (ft). The data from both PNNL and PSU had gage heights obtained in NAVD 88 in meters. A conversion factor of 3.281 was used to convert data from meters to feet. NOAA tidal stage data were obtained in Station Datum (STND), an arbitrarily established fixed base elevation, and are converted to NAVD 88 using conversion values from Bondurant (2019). WADOE gage height data were referenced to an arbitrary datum and left as is. USGS data were found in multiple datums: Columbia River Datum (CRD), NGVD 29, and NAVD 88. Data in CRD and NGVD 29 were converted to NAVD 88 using VERTCON 2.0 software downloaded from National Oceanic and Atmospheric Administration (2018b). The values determined by VERTCON 2.0 are supplied in table 14. 
Table 14. Conversion values used to adjust gage datums to North American Vertical Datum of 1988.

[Abbreviations: CRD, Columbia River Datum; ft, feet; NA, not applicable; NAVD 88, North American Vertical Datum of 1988; NGVD 29, National Geodetic Datum of 1929; NOAA, National Oceanic and Atmospheric Administration; OR, Oregon; St, street; USGS, U.S. Geological Survey; WA, Washington]

\begin{tabular}{|c|c|c|c|c|c|c|c|}
\hline Operator & $\begin{array}{l}\text { Station } \\
\text { identifier }\end{array}$ & Station name & Original station datum & $\begin{array}{l}\text { Conversion to } \\
\text { NGVD } 29 \text { if } \\
\text { needed (in feet) }\end{array}$ & $\begin{array}{l}\text { VERTCON conversion } \\
\text { factor from NGVD } 29 \\
\text { to NAVD } 88 \text { (in feet) }\end{array}$ & $\begin{array}{l}\text { Conversion from } \\
\text { gage datum to } \\
\text { NAVD } 88 \\
\text { (in feet) }\end{array}$ & Notes \\
\hline USGS & 14144700 & $\begin{array}{r}\text { Columbia River at } \\
\text { Vancouver, WA }\end{array}$ & CRD & Add 1.82 & 3.45 & 5.27 & $\begin{array}{l}2018 \text { resurvey of USGS } \\
\text { gage. CRD to NAVD } \\
88 \text { is } 5.26^{\mathrm{a}}\end{array}$ \\
\hline USGS & 14197900 & $\begin{array}{l}\text { Willamette River at } \\
\text { Newberg, OR }\end{array}$ & NGVD 29 & NA & 3.38 & 3.38 & USGS gage notes \\
\hline USGS & 14211010 & $\begin{array}{c}\text { Clackamas River at } \\
\text { Oregon City. OR }\end{array}$ & NGVD 29 & NA & 3.47 & 3.47 & USGS gage notes \\
\hline USGS & 14211550 & $\begin{array}{r}\text { Johnson Creek at } \\
\text { Milwaukie, OR }\end{array}$ & NGVD 29 & NA & 3.47 & 3.47 & USGS gage notes \\
\hline USGS & 14211720 & $\begin{array}{l}\text { Willamette River at } \\
\text { Portland, OR }\end{array}$ & $\begin{array}{l}1.48 \mathrm{ft} \text { above NGVD } \\
29^{2}\end{array}$ & Add 1.48 & 3.48 & 4.96 & $\begin{array}{c}2018 \text { resurvey of USGS } \\
\text { gage. Agrees with } \\
\text { Bondurant (2019). }\end{array}$ \\
\hline USGS & 14246900 & $\begin{array}{l}\text { Columbia River at } \\
\text { Port Westward, } \\
\text { near Quincy, OR }\end{array}$ & CRD & Subtract 1.00 & 3.07 & 2.07 & $\begin{array}{l}\text { USGS gage notes. } \\
\text { USACE identified } \\
\text { uncertainty in gage } \\
\text { datum before October } \\
1,2006 \text {. }\end{array}$ \\
\hline USGS & 14211820 & $\begin{array}{l}\text { Columbia Slough at } \\
\text { Portland, OR }\end{array}$ & $\begin{array}{l}1.53 \mathrm{ft} \text { above NGVD } \\
29\end{array}$ & Add 1.53 & 3.39 & 4.92 & USGS gage notes \\
\hline USGS & 14207740 & $\begin{array}{l}\text { Willamette River } \\
\text { above Falls at } \\
\text { Oregon City, OR }\end{array}$ & NGVD 29 & NA & 3.5 & 3.5 & USGS gage notes \\
\hline USGS & 14207770 & $\begin{array}{l}\text { Willamette River } \\
\text { below Falls, at } \\
\text { Oregon City, OR }\end{array}$ & NGVD 29 & NA & 3.49 & 3.49 & USGS gage notes \\
\hline USGS & 14128870 & $\begin{array}{l}\text { Columbia River } \\
\text { below Bonneville } \\
\text { Dam, OR }\end{array}$ & NGVD 29 & NA & 3.34 & 3.34 & USGS gage notes \\
\hline USGS & 14128910 & $\begin{array}{r}\text { Columbia River at } \\
\text { Warrendale, OR }\end{array}$ & NGVD 29 & NA & 3.35 & 3.35 & USGS gage notes \\
\hline
\end{tabular}




\begin{tabular}{|c|c|c|c|c|c|c|c|}
\hline Operator & $\begin{array}{l}\text { Station } \\
\text { identifier }\end{array}$ & Station name & Original station datum & $\begin{array}{l}\text { Conversion to } \\
\text { NGVD } 29 \text { if } \\
\text { needed (in feet) }\end{array}$ & $\begin{array}{l}\text { VERTCON conversion } \\
\text { factor from NGVD } 29 \\
\text { to NAVD } 88 \text { (in feet) }\end{array}$ & $\begin{array}{l}\text { Conversion from } \\
\text { gage datum to } \\
\text { NAVD } 88 \\
\text { (in feet) }\end{array}$ & Notes \\
\hline USGS & 14222880 & $\begin{array}{l}\text { Columbia River at } \\
\text { Columbia City, } \\
\text { OR }\end{array}$ & $\begin{array}{l}0.79 \mathrm{ft} \text { above NGVD } \\
29\end{array}$ & Add 0.79 & 3.33 & 4.12 & USGS gage notes \\
\hline NOAA & 9439040 & Astoria, OR & Station datum & NA & 3.37 & -2.02 & $\begin{array}{l}\text { NOAA Tides and } \\
\text { Currents (present-day) }\end{array}$ \\
\hline NOAA & 9440569 & Skamokawa, WA & $\begin{array}{l}\text { Station datum }(0.06 \mathrm{ft} \\
\text { above CRD })^{3}\end{array}$ & NA & 3.1 & 1.01 & $\begin{array}{l}\text { CRD to NAVD } 88 \text { is } \\
{ }^{1} 0.95\end{array}$ \\
\hline NOAA & 9439099 & Wauna, OR & $\begin{array}{l}\text { Station datum }(0.14 \mathrm{ft} \\
\text { above CRD })^{3}\end{array}$ & NA & 3.5 & 1.62 & $\begin{array}{l}\text { CRD to NAVD } 88 \text { is } \\
{ }^{1} 1.48\end{array}$ \\
\hline NOAA & 9440422 & Longview, WA & $\begin{array}{l}\text { Station datum }(0.27 \mathrm{ft} \\
\text { below CRD })^{3}\end{array}$ & NA & 3.2 & 2.59 & $\begin{array}{l}\text { CRD to NAVD } 88 \text { is } \\
{ }^{2} 2.86\end{array}$ \\
\hline NOAA & 9439201 & St. Helens, OR & $\begin{array}{l}\text { Station datum }(0.02 \mathrm{ft} \\
\text { below CRD })^{3}\end{array}$ & NA & 3.3 & 4.13 & $\begin{array}{l}\text { CRD to NAVD } 88 \text { is } \\
{ }^{4} 4.15\end{array}$ \\
\hline NOAA & 9440083 & Vancouver, WA & $\begin{array}{l}\text { Station datum }(0.17 \mathrm{ft} \\
\text { above CRD })^{3}\end{array}$ & NA & 3.44 & 5.31 & $\begin{array}{l}\text { CRD to NAVD } 88 \text { is } \\
\quad 15.15\end{array}$ \\
\hline NOAA & 9439221 & $\begin{array}{l}\text { Portland Morrison } \\
\text { St Bridge, OR }\end{array}$ & $\begin{array}{l}\text { Station datum (likely } \\
\text { CRD) }\end{array}$ & NA & 3.48 & 5.38 & $\begin{array}{l}\text { CRD to NAVD } 88 \text { is } \\
{ }^{15.38}\end{array}$ \\
\hline
\end{tabular}

${ }^{1}$ CRD to NAVD 88 values determined by Bondurant (2019).

${ }^{2}$ Previously noted as $1.55 \mathrm{ft}$ above NGVD 29 before USGS resurvey in 2018.

${ }^{3}$ Value above or below CRD determined from NOAA website (National Oceanic and Atmospheric Association, 2018a). 
Of the 12 USGS stage datasets compiled for this study, only Cowlitz River at Castle Rock, WA (14243000), needed additional adjustments. The stage data at 14243000 were collected in NAVD 88 for most of the record except for the period of May 23, 1980, to June 29, 1997, where the USGS datum notes stated the water-stage recorder was at present site at datum, $23.65 \mathrm{ft}$ higher. For the period in question, $23.65 \mathrm{ft}$ was added to the gage datum to create a continuous NAVD 88 dataset.

\section{Data Review by Data Source}

Stage and streamflow data were scanned for erroneous periods and data were removed when deemed necessary. Erroneous data, often referred to as "data spikes," were removed based on hydrologic judgement. Nearby stations, if available, were also used to support the decision to remove the data spikes. The following sections detail the edits made, if needed, to each of the sources of data compiled for this study.

\section{U.S. Geological Survey (USGS)}

Historically, the USGS published streamflow data but not stage data. Because stage data were not published and were only used as an intermediate step in the calculation of streamflow, historical stage records did not undergo the same level of editorial clean-up and may contain some erroneous data. Erroneous data could have resulted from instances where the equipment used to measure stage failed or due to environmental conditions. If this occurred, the streamflow data may have been estimated or calculated without the use of the standard relationship between stage and streamflow. In such instances, the stage data might not reflect the true stage of the stream for that period-of-time. Specific edits to records are detailed below.

For the 1974 flooding event, Butte Creek at Monitor, OR (14201500), stage data, used to compute the sub-daily streamflow record, contained a string of repetitive stage values on the recession of the streamflow flooding event peak, suggesting the stage data were erroneous. It was determined this was the result a stage recorder malfunction and the corresponding streamflow data were removed. The 1982 flooding event at Tualatin River near Gaston, OR (14202500), also had signs of a stage recorder malfunction on the recession of the streamflow flooding event peak. The corresponding streamflow during the time of stage recorder malfunction was also removed from the record.

Erroneous stage-data spikes were removed from the record in 1990, 1991, 1992, and 1998 at Columbia River below Bonneville Dam (14128870). Cowlitz River at Castle Rock (14243000) had erroneous stage data spikes removed from the record in the following years: 1989, 1990, and 1995. For the stage data at Willamette River above the Falls, at Oregon City (14207740), the stage recorder appeared to be failing during two periods of data, one in 1991 and one in 1994, the data were removed from the record. Erroneous data points were removed from the stage record at Willamette River below the Falls, at Oregon City (14207770) in 1990, 1994, and 1996.

Due to changes in rounding values over time, as well as the subjective process of visual interpretation of stage values from paper tape chart line plots, the recreated sub-daily streamflow data for flooding events may not match the historically computed daily records. Stage values were physically read off the paper tape chart line plots, so a certain degree of uncertainty could be related to those stage values "picked off the line" and the corresponding streamflow. 
Oregon Water Resources Department (OWRD)

OWRD data were reviewed for erroneous data. No edits were deemed necessary.

National Oceanic and Atmospheric Administration (NOAA)

NOAA tidal data were reviewed for erroneous data. No edits were deemed necessary.

Washington Department of Ecology (WA-DOE)

Data from WA-DOE were reviewed for erroneous data, and edits were made as deemed necessary. Data collection during high flow events is not the primary mission of WA-DOE. Therefore, field measurements are often not taken during high flow events, reducing the accuracy of the rating curves and flow data for large events. Edits and review documentation are detailed below broken into sub-sections for stage and streamflow. WA-DOE provided a list of defined codes that were used when qualifying the stage and streamflow data (J. Shedd, WADOE, written communication, May 11, 2020). These codes were considered when analyzing the data for data edits.

Stage

The early portion of data from Chinook River at Chinook Valley Road (24N070), collected from December 2014 to July 2015 and from August to October 2015, diverged significantly from the rest of the record. After an email response from WA-DOE staff, it was determined that during this period the tide gates were fully raised so that a contractor could install a lid on a culvert on highway 101. During this period, the gage infrastructure was modified so that all tidal changes would be recorded (J. Shedd, WA-DOE, written commun., July 13 , 2018). During some periods of 2015, missing data had been coded as "below instrument threshold" by WA-DOE.

From October 2005 to June 2006, there was a large portion of data missing from Abernathy Creek near Mouth (25E060). These data had been coded by WA-DOE as "estimated data-unreliable" and were therefore removed from the record by WA-DOE. The stage data during low flow periods prior to June 2006 appears lower than the stage data after it. The station had been moved to a new location on June 28, 2006, which accounted for this change in the stage values observed. No adjustments were made to the stage data to account for the station moving.

At Mill Creek at Mouth (25F060), two periods (May 14,2011-July 19, 2011 and March 31, 2012-May 9, 2012) were coded by WA-DOE as "backwater flow conditions" and, therefore, no data were reported by WA-DOE. From March to June 2017, stage data differ from the surrounding periods with a more pronounced oscillation. The Mill Creek stage data were plotted against a nearby site, Coweeman River at Kelso (26C075), and the oscillations were not observed there. The data were coded as "good quality provisional data" by WA-DOE. This period also coincides with backwater conditions when the Columbia River stage was high. To stay consistent with previous periods where WA-DOE had removed backwater effected areas these data were also removed.

In February 2012 a period of data that had been coded as "questionable estimate" by WA-DOE was removed from the dataset at Washougal River at Hatahway Park (28B080). It deviated from the general trend, and similar patterns were not observed at nearby sites Coweeman River at Kelso and Mill Creek at Mouth. Additional periods of missing data were noted in March 2012; there were two periods coded by WA-DOE as "instrument impaired," but 
the data in-between was coded as "good quality provisional data." The "good quality provisional data" did not follow the trends of nearby sites and was removed as potentially suspect.

\section{Streamflow}

Chinook River near Houchen Street (24N100) had a few streamflow peaks which appeared to be outliers. During these periods, December 2014, January 2015, and November 2016, the data were coded by WA-DOE as "above rating, over $2 x$." The data were compared with a nearby USGS gage, Naselle River near Naselle, WA (12010000), these higher peaks corresponded to similar peaks of differing magnitudes at the USGS gage and were therefore kept in the record.

Some high streamflow peaks were also observed at Grays River near Mouth (25B060) during 2006-09. For the periods in question the data were coded by WA-DOE as "above rating, over 2x." The data were again compared with USGS gage 12010000 with similar corresponding peaks, of differing magnitude, being present. Notes were also found in WA-DOE documentation that USGS gage Chehalis River near Doty, WA (12020000), stage data had occasionally been used to compute streamflow when stage data were missing at Grays River near Mouth. The streamflow at 12020000 was found to have similar high flow peaks supporting the peaks at 25B060, and they remain in the record. High flow peaks were not present from the end of 2009 through 2013 as the data had been deleted by WA-DOE with the data being coded as "estimated data - unreliable."

The following sites had minor data gaps throughout the period of record: Elochoman River at Monroe Drive (25C060), Germany Creek at Mouth (25D050), Abernathy Creek near Mouth (25E060), Mill Creek at Mouth (25F060), EF Lewis near Dollar Corner (27D090), Rock Creek at Stevenson (29A070), and Wind River at Stabler (29C070). These gaps were associated with data flags of "rating table exceeded (data will not be reported)," "incomplete day," or "estimated data - unreliable." Mill Creek at Mouth also had a few small gaps in 2011 and 2012 associated with a data flag of "backwater flow conditions."

Coweeman River at Kelso (26C075) had streamflow peaks which appeared to be outliers during 2006-09. These peaks corresponded to data periods flagged as "above rating, over $2 \mathrm{x}$ " by WA-DOE. A nearby USGS gage, Cowlitz River at Castle Rock, WA (14243000), was used as a comparison site and found corresponding similar peaks, at differing magnitudes, were present. This supported keeping the peaks at 26C075 in the record.

Washougal River at Hathaway Park (28B080) had a combination of some higher flows in 2006 and 2009 associated with data coded as "modeled flow-calibrated results exceed 20 percent of measured flow." A nearby USGS gage, Sandy River near Marmot, OR (14137000), was used for comparison and displayed similar high flow peaks of differing magnitudes, this provided the support for keeping the peaks in the record. Due to not knowing the methods WA-DOE uses to model flow at this station, calculated streamflow values were assumed accurate and retained. Minor periods of missing data were associated with data coded as either "rating table exceeded (data will not be reported)," "incomplete day," "bankfull discharge exceeded," or "estimated data-unreliable."

A few periods of high flow were observed at Little Klickitat near Wahkiacus (30C070) in 2007, 2009, 2011, 2012, and 2017. These are all associated with data coded as "modeled flow." Data from 2017 were also coded as "estimated from another station, same variable." Similar high flow peaks at the USGS gage Klickitat River near Pitt, WA (14113000), were found to correspond, at differing magnitudes, to the higher flows observed at 30C070. This provided the 
support for keeping the higher flows for 30C070 in the record. Various data throughout the period were missing and associated to codes "instrument impaired," "rating table exceeded," and "ice impacted data."

\section{Pacific Northwest National Laboratory (PNNL)}

The following stations had erroneous points, in the form of data spikes, that were removed from the elevation records using hydrologic judgement: Baker Bay Marsh, Bradwood Slough Marsh, Burke Island Marsh, Clatskanie River Marsh, Cunningham Lake Marsh, Fort Clatsop Dike-Breach Marsh, Gee Creek Riparian Wetland, Karlson Island Dike-Breach Marsh, Reed Island Slough Marsh, Sauvie Willow Bar Marsh, Walluski River Dike-Breach Marsh, Walluski River Marsh, Welch Island Marsh Slough, and Whites Island Marsh. Stations were compared to others in the area for evaluating when to remove spikes.

Data were typically recorded in 30-minute or 1-hour increments, and typically recorded on the hour. Three stations had hourly data but on slightly differing recording marks: Chinook River Marsh (CHM-HOBO) on the 20-minute mark, Lord Island Marsh 2 (LI2-HOBO) on the 45-minute mark, and Walluski River Dike-Breach Marsh (WRB-HOBO) on the 14-minute mark. Times were not adjusted for these stations.

The snap to time function was used within the HEC-DSS software when the data files from PNNL appeared to have a slight time offset during various deployment periods. This allowed the irregular time series to be adjusted to a regular time series. The time shifts were anywhere from 1 to 13 minutes. The following sites had time shifts applied: Crooked Creek Swamp (CCS-HOBO), Goat Island Marsh (GIC-HOBO), and Seal Slough Swamp (SSSHOBO2). The first deployment period for the Secret River Marsh (SRM-HOBO) was set to 30minute data. and all other deployments were set to 1 hour. The 30-minute data were snapped to hourly data to create a regular complete final dataset at a 1-hour interval. The original 30-minute dataset was retained as an unaltered file.

Portland State University (PSU)

Data provided by PSU had been reviewed by their staff and were assigned a quality assurance level of "good," based on a qualitative scale of "light," "good," or "excellent." Datasets were qualitatively reviewed for this study, and no edits were deemed necessary.

The Portland water-level data provided by PSU was collected on the Willamette River in downtown Portland. This data covers several staff gage locations over time and does not have a defined station identifier. Table 15 lists the time periods and locations of the Portland staff gage (Bondurant, 2019).

Table 15. Staff gage locations over time in Portland, Oregon.

\begin{tabular}{llrrl}
\hline \multicolumn{1}{c}{ Time frame } & \multicolumn{1}{c}{ Staff gage location } & Latitude & Longitude & Time frame \\
\hline $1876-96$ & Stark Street Bridge & $45^{\circ} 31^{\prime} 09^{\prime \prime}$ & $122^{\circ} 40^{\prime} 14^{\prime \prime}$ & $1876-1896$ \\
$1896-05$ & 1st Morrison Street Bridge & $45^{\circ} 31^{\prime} 02^{\prime \prime}$ & $122^{\circ} 40^{\prime} 18^{\prime \prime}$ & $1896-1905$ \\
$1905-58$ & 2nd Morrison Street Bridge & $45^{\circ} 31^{\prime} 05^{\prime \prime}$ & $122^{\circ} 40^{\prime} 16^{\prime \prime}$ & $1905-1958$ \\
$1958-$ Present & 3rd Morrison Street Bridge & $45^{\circ} 31^{\prime} 03^{\prime \prime}$ & $122^{\circ} 40^{\prime} 09^{\prime \prime}$ & $1958-$ Present \\
\hline
\end{tabular}




\section{U.S. Army Corps of Engineers (USACE)}

Data provided by USACE via Dataquery files in HEC-DSS format were typically in local standard time. Some data supplied by USACE from Dataquery were found to have stage values off exactly by a factor of 10 . In such instances, stage values were divided by 10 to match the stage from periods either before or after the period in question. USACE staff confirmed (R. Cahill, USACE, written commun., October 18, 2018) this approach. Erroneous data points were removed from the supplied records using hydrologic judgement and by comparing data from nearby locations. The following stations each had some data where this manipulation was needed to correct the data back to within reason: Bonneville (BON), Columbia at Columbia City (COCO), Columbia River at Camas (CAMW), Wauna (WAUO/9439099), Willamette River above the falls, at Oregon City (ORC/14207740), and Willamette River below the falls, at Oregon City (ORCO/14207770).

\section{Combining Data from Multiple Sources}

On a few occasions, data from the same station were available from multiple data sources (table 16). When possible, the data were combined to extend the dataset and form a continuous period of record. The primary and secondary data sources are listed in table 13. In all instances, the primary data source is presented in Pacific Standard Time and the secondary data source is in local standard time. These 1-hour discrepancies in time were deemed to be inconsequential for the purpose of this project compilation; therefore, no time adjustments were made to the secondary data source.

Table 16. Gaging stations where data were combined from a secondary data source to extend the record or fill in some missing time periods.

[Abbreviations: NOAA, National Oceanic and Atmospheric Administration; OR, Oregon; USACE, U.S. Army Corps of Engineers; USGS, U.S. Geological Survey; WA, Washington]

\begin{tabular}{lcccc}
\hline \multicolumn{1}{c}{ Gaging station name } & $\begin{array}{c}\text { Primary } \\
\text { data } \\
\text { source }\end{array}$ & $\begin{array}{c}\text { Primary } \\
\text { station } \\
\text { identifier }\end{array}$ & $\begin{array}{c}\text { Secondary data } \\
\text { source }\end{array}$ & $\begin{array}{c}\text { Secondary } \\
\text { station } \\
\text { identification }\end{array}$ \\
\hline Willamette River at Portland, OR & USGS & 14211720 & Dataquery USACE & PRTO \\
Columbia River at Vancouver, WA & USGS & 14144700 & Dataquery USACE & VANW \\
Cowlitz River at Castle Rock, WA & USGS & 14243000 & Dataquery USACE & CASW \\
Willamette River above Falls, at Oregon City, OR & USGS & 14207740 & Dataquery USACE & ORC \\
Willamette River below Falls, at Oregon City, OR & USGS & 14207770 & Dataquery USACE & ORCO \\
Longview, WA & NOAA & 9440422 & Dataquery USACE & LONW \\
St. Helens, OR & NOAA & 9439201 & Dataquery USACE & SHNO \\
Vancouver, WA & NOAA & 9440083 & Dataquery USACE & VAPW \\
Wauna, OR & NOAA & 9439099 & Dataquery USACE & WAUO \\
\hline
\end{tabular}

The elevation data were combined at the Willamette River at Portland, OR, gage between USACE Dataquery (PRTO) and USGS (14211720) data files. The Dataquery file was used exclusively from 14:00 on October 1, 1976, to 18:00 on September 30,1988, to extend the station dataset further back in time from where the USGS data began. An observable time difference of approximately 10 hours with the Dataquery file from October 1976 to December 1992 was visible in the overlapping portions of the two datasets. During four additional time periods where USGS data were currently unavailable, the Dataquery files were used to fill in missing periods: January-April 1993, July-September 1994, March-May 1995, and November 
1995. During these time periods, the data were found to coincide well with only the slight 1-hour time difference noted.

Columbia River at Vancouver elevation data were extended as a combination of Dataquery (VANW) and USGS (14144700) files. There was a small period of overlap between the two datasets where the time datum lined up. Dataquery files from 15:00 on November 1, 1974, to 15:00 on February 4, 1998, were used to extend the NOAA dataset back further in time.

For the Cowlitz River at Castle Rock, WA, gage, a Dataquery file was used for the early portion of the elevation dataset from $13: 30$ on October 31,1988 , to $12: 15$ on July 29 , 1997. The remainder of the dataset from 12:30 on July 29, 1997, to present is from the USGS.

Willamette River above the Falls, at Oregon City USGS (14207740), elevation data were extended further back in time with a single Dataquery (ORC) file. The Dataquery file from 15:00 on November 1, 1974, to 23:00 on September 30,1986, was used directly as received from USACE. The remainder of the dataset from 24:00 on September 30, 1986, to present, is from the USGS.

Willamette River below the Falls, at Oregon City USGS (14207770), elevation data were extended further back in time with two additional Dataquery (ORCO) files. The first Dataquery file from 14:00 on October 1, 1976, to 15:00 on March 31, 1980, was used directly as received from USACE. The second Dataquery file was off by a factor of 10 and needed to be divided by 10 to match the time periods both before and after it. The second Dataquery file covered the period from 14:00 on April 1, 1980, to 14:00 on September 30, 1988. The remainder of the dataset from 24:00 on September 30, 1988, to present is from the USGS.

The following NOAA tidal elevation gages were combined with additional data provided by USACE Dataquery: Longview (LONW/9440422), Vancouver (VAPW/9440083), Wauna (WAUO/9439099), and St Helens (SHNO/9439201).

The Longview gage dataset was extended back in time with a Dataquery file from 15:00 on November 1, 1974, to 19:46 on March 26, 2002. The NOAA data covers the period from 20:00 on March 26, 2002, to present.

Two Dataquery files were used to extend the St Helens gage dataset. The first file from 24:00 on March 10, 1986, to 24:00 on December 31, 1990, and the second file from 01:00 on January 1, 1991, to 14:00 on March 26, 2002. The second Dataquery file continued to overlap with the original NOAA data file through May 2004. There were varying discrepancies noted in both the elevation values as well as the time datum shifts for the second Dataquery file, these were left as is. The NOAA data covers the period from 17:00 on March 26, 2002, to present.

The Wauna gage was extended with two additional Dataquery files. A combination of the first and second Dataquery files, from 24:00 on March 10, 1986, to 16:54 on March 26, 2002, were used to extend the NOAA period further back in time. Two portions of the second Dataquery file were used to fill in two sections, from 10:06 on April 17, 2003, to 18:00 on March 4, 2004, and from 10:00 on November 23, 2004, to 12:00 on June 3, 2005, which were missing from the NOAA dataset.

The Vancouver gage was extended with one additional Dataquery file. The first period, from 24:00 on March 10, 1986, to 19:00 on March 26, 2002, was used to extend the NOAA dataset back in time. The second portion of the Dataquery file was used to fill in one section, from 12:00 on December 2, 2004, to 16:00 on May 31, 2005, which was missing from the NOAA dataset.

Seven gages are currently being operated by OWRD after being previously operated by USGS. Those datasets were combined between the two sources to form a continuous record for 
both daily mean streamflow and the annual peak streamflow at the gages involved. Table 17 details the USGS gages now operated by OWRD and the corresponding time periods of each agency's operation of the gage.

Table 17. Current Oregon Water Resources Department gaging stations that were previously run by U.S. Geological Survey.

[All stations were still active at time of data compilation. Dates USGS operated and Dates OWRD operated periods may not have been continuous. Abbreviations: Ab, above; Bl, below; Cr, creek; Nr, near; Or, Oregon; OWRD, Oregon Water Resources Department; R, river; USGS, U.S. Geological Survey]

\begin{tabular}{|c|c|c|c|c|c|}
\hline $\begin{array}{l}\text { Station } \\
\text { identifier }\end{array}$ & Station name & Latitude & Longitude & $\begin{array}{l}\text { Dates USGS } \\
\text { operated }\end{array}$ & $\begin{array}{l}\text { Dates OWRD } \\
\text { operated }\end{array}$ \\
\hline 14192500 & $\begin{array}{c}\text { South Yamhill R Nr } \\
\text { Willamina, OR }\end{array}$ & $45^{\circ} 2^{\prime} 50.712^{\prime \prime}$ & $-123^{\circ} 30^{\prime} 15.451^{\prime \prime}$ & $\begin{array}{l}\text { May 1, 1934- } \\
\text { September 29, } \\
1993\end{array}$ & $\begin{array}{l}\text { September 30, } \\
\text { 1993-March 1, } \\
2018\end{array}$ \\
\hline 14193000 & $\begin{array}{r}\text { Willamina Cr Nr } \\
\text { Willamina, OR }\end{array}$ & $45^{\circ} 8^{\prime} 35.671^{\prime \prime}$ & $-123^{\circ} 29^{\prime} 40.7^{\prime \prime}$ & $\begin{array}{l}\text { June } 1,1934- \\
\text { October 29, } \\
1991\end{array}$ & $\begin{array}{l}\text { September 30, } \\
\text { 1991-March 1, } \\
2018\end{array}$ \\
\hline 14194300 & $\begin{array}{l}\text { North Yamhill R Nr } \\
\text { Fairdale, OR }\end{array}$ & $45^{\circ} 21^{\prime} 53.852^{\prime \prime}$ & $-123^{\circ} 22^{\prime} 45.48^{\prime \prime}$ & $\begin{array}{l}\text { October 1, 1958- } \\
\text { October 29, } \\
1991\end{array}$ & $\begin{array}{l}\text { September 30, } \\
\text { 1991-March 1, } \\
2018\end{array}$ \\
\hline 14202850 & $\begin{array}{l}\text { Scoggins Cr Ab } \\
\text { Henry Hagg Lake } \\
\text { Nr Gaston, OR }\end{array}$ & $45^{\circ} 29^{\prime} 59.791^{\prime \prime}$ & $-123^{\circ} 15^{\prime} 4.277^{\prime \prime}$ & $\begin{array}{l}\text { October 1, 1972- } \\
\text { October 29, } \\
1976\end{array}$ & $\begin{array}{l}\text { September 30, } \\
\text { 1976-March 1, } \\
2018\end{array}$ \\
\hline 14202920 & $\begin{array}{l}\text { Sain Cr Nr Gaston, } \\
\text { OR }\end{array}$ & $45^{\circ} 28^{\prime} 50.891^{\prime \prime}$ & $-123^{\circ} 14^{\prime} 45.19^{\prime \prime}$ & $\begin{array}{l}\text { October 1, 1972- } \\
\text { October 29, } \\
1976\end{array}$ & $\begin{array}{l}\text { September 30, } \\
\text { 1976-March 1, } \\
2018\end{array}$ \\
\hline 14202980 & $\begin{array}{l}\text { Scoggins Cr Bl } \\
\text { Henry Hagg Lake } \\
\text { Nr Gaston, OR }\end{array}$ & $45^{\circ} 28^{\prime} 18.322^{\prime \prime}$ & $-123^{\circ} 11^{\prime} 50.881^{\prime \prime}$ & $\begin{array}{l}\text { January 1, 1975- } \\
\text { October 1, } \\
2006\end{array}$ & $\begin{array}{r}\text { October 2, 2006- } \\
\quad \text { March 1, } 2018\end{array}$ \\
\hline 14206500 & $\begin{array}{l}\text { Tualatin R at } \\
\text { Farmington, OR }\end{array}$ & $45^{\circ} 26^{\prime} 57.71^{\prime \prime}$ & $-122^{\circ} 57^{\prime} 2.588^{\prime \prime}$ & $\begin{array}{l}\text { October 1, 1939- } \\
\text { October 29, } \\
1976\end{array}$ & $\begin{array}{l}\text { September } 30 \text {, } \\
\text { 1976-January } \\
25,2018\end{array}$ \\
\hline
\end{tabular}

\section{Maximum Stage and Streamflow Statistics}

$\mathrm{R}$ was used to compute each gage's maximum stage and streamflow statistics. Water-year (October 1-September 30) maximum stage and streamflow were computed. Seasonal maximum statistics were computed for the typical high-flow seasons of the LCR basin, winter (NovemberMarch) and spring (April-July). Typical low-flow season (summer-autumn) statistics were not computed for this study. All maximum statistic results are available at Boudreau and others (2021).

\section{Supplemental Information}

In addition to aggregating a comprehensive streamflow and elevation dataset for the study area, additional analysis was provided for three USGS stations within the study area. These stations are briefly summarized here and available as Appendix 1 and 2 below.

The USGS does not have an established rating for computing streamflow at Willamette River above Falls, at Oregon City (14207740). Appendix 1 describes the history and reasoning 
behind why continuous streamflow has not been part of the historical record at the gage and discusses two methods that could be used to produce a record of continuous streamflow.

An evaluation of the gage data at Willamette River at Portland (14211720) and Columbia River at Vancouver (14144700) was completed along with a re-survey at each gage location to associated benchmarks (Appendix 2). The USGS gage datum at 14211720, which had previously been described as $1.55 \mathrm{ft}$ above NGVD29, has since been adjusted to $1.48 \mathrm{ft}$ above NGVD29 or $4.96 \mathrm{ft}$ above NAVD88. The vertical gage datum at 14144700 is CRD, and the reported conversion was previously described as $1.82 \mathrm{ft}$ above NGVD29. This datum has since been updated to $1.77 \mathrm{ft}$ above NGVD29 or $5.22 \mathrm{ft}$ above NAVD88.

\section{Summary}

A total of 402 datasets from 7 national and state agencies were compiled into a HECDSSVue database file. These included daily streamflow, annual peak streamflow, streamflow from various historic flooding events, along with elevation and stage data. The datasets were reviewed for any potential erroneous data before being accepted into the final database. The database files are available at Boudreau and others (2021).

\section{References Cited}

Bondurant, C.L., 2019, Lower Columbia and Willamette Rivers elevation datum summary:

Portland, Oregon, U.S. Army Corps of Engineers, 45 p. [This is an internal document available by request from the U.S. Army Corps of Engineers.]

Boudreau, C.L., Stewart, M.A., and Stonewall, A.J., 2021, Historical Streamflow and Stage Data for the Lower Columbia River Basin and the Coasts of Washington, Oregon, and Northern California: U.S. Geological Survey data release, https://doi.org/10.5066/P9R6RT0Z.

Burgette, R.J., Weldon II, R.J., and Schmidt, D.A., 2009, Interseismic uplift rates for western Oregon and along-strike variation in locking on the Cascadia subduction zone: Journal of Geophysical Research, v. 114, B01408, 24 p., doi:10.1029/2008JB005679.

Columbia Basin Inter-Agency Committee, 1966, Report on summary of operations Columbia River 1965 flood: Portland, Oregon, 18 p.

National Oceanic and Atmospheric Administration, 2018a, NOAA tides \& currents: National Oceanic and Atmospheric Administration web page, accessed March 16, 2018, at https://tidesandcurrents.noaa.gov/stations.html.

Northwest Hydraulic Consultants, Ogden Beeman \& Associates, and Oregon Climate Service, 1997, February 1996 postflood report-Hydrometeorological evaluation: U.S. Army Corps of Engineers, $101 \mathrm{p}$.

Oregon Water Resources Department, 2018a, Oregon water resources department: State of Oregon web page, accessed January 26, 2018, at https://www.oregon.gov/owrd/Pages/index.aspx.

Oregon Water Resources Department, 2018b, Oregon water resources department historical streamflow and lake level data: State of Oregon web page, accessed January 26, 2018, at https://apps.wrd.state.or.us/apps/sw/hydro_report/.

Oregon Water Resources Department, 2018c, Oregon water resources department near real time hydrographics data: State of Oregon web page, accessed January 26, 2018, at https://apps.wrd.state.or.us/apps/sw/hydro_near_real_time/. 
Talke, S.A., and Jay, D.A., 2017, Archival water-level measurements-Recovering historical data to help design for the future: Civil Work technical series, Report CWTS-02, $50 \mathrm{p}$.

U.S. Army Corps of Engineers, 1943, The Willamette River Flood, December 31, 1942-January 4, 1943: Portland, Oregon, 35 p.

U.S. Army Corps of Engineers, 1949, Report on Flood of May-June 1948-Columbia River and Tributaries below Yakima River: U.S. Army Corps of Engineers, 167 p.

U.S. Army Corps of Engineers, 1956, Western Oregon streams report on floods of December 1955: U.S. Army Corps of Engineers, 85 p.

U.S. Army Corps of Engineers, 1957, Report on flood of May-June 1956-Columbia River and tributaries below Yakima River: U.S. Army Corps of Engineers, 44 p.

U.S. Army Corps of Engineers, 1963, Report on flood of February 1961, Willamette and Umpqua River Basins: U.S. Army Corps of Engineers, 24 p.

U.S. Army Corps of Engineers, 1966, Postflood report-December 1964, January 1965 flood: U.S. Army Corps of Engineers, 237 p.

U.S. Army Corps of Engineers, 1975, Postflood report-January 1974: U.S. Army Corps of Engineers, $175 \mathrm{p}$.

U.S. Army Corps of Engineers, 1991, Review of flood control, Columbia River Basin: Portland, Oregon, Columbia River and tributaries study, CRT-63, $135 \mathrm{p}$.

U.S. Army Corps of Engineers, 2018b, Dataquery 2.0-Query timeseries from USACE Northwestern division: U.S. Army Corps of Engineers, web page, accessed May 24, 2018, at http://www.nwd-wc.usace.army.mil/dd/common/dataquery/www/.

U.S. Army Corps of Engineers, 2018a, Hydrologic engineering center-HEC-DSSVue: U.S. Army Corps of Engineers, web page, accessed Jan. 26, 2018, at https://www.hec.usace.army.mil/software/hec-dssvue/.

U.S. Geological Survey, 2018, National Water Information System-Water data for the nation: U.S. Geological Survey database web page, accessed March 6, 2018, at http://dx.doi.org/10.5066/F7P55KJN.

Washington State Department of Ecology, 2018, River and stream flow monitoring-Flow monitoring network: Washington State Department of Ecology web page, accessed March 5, 2018, at https://fortress.wa.gov/ecy/eap/flows/regions/state.asp?stationfilter=1\&region. 


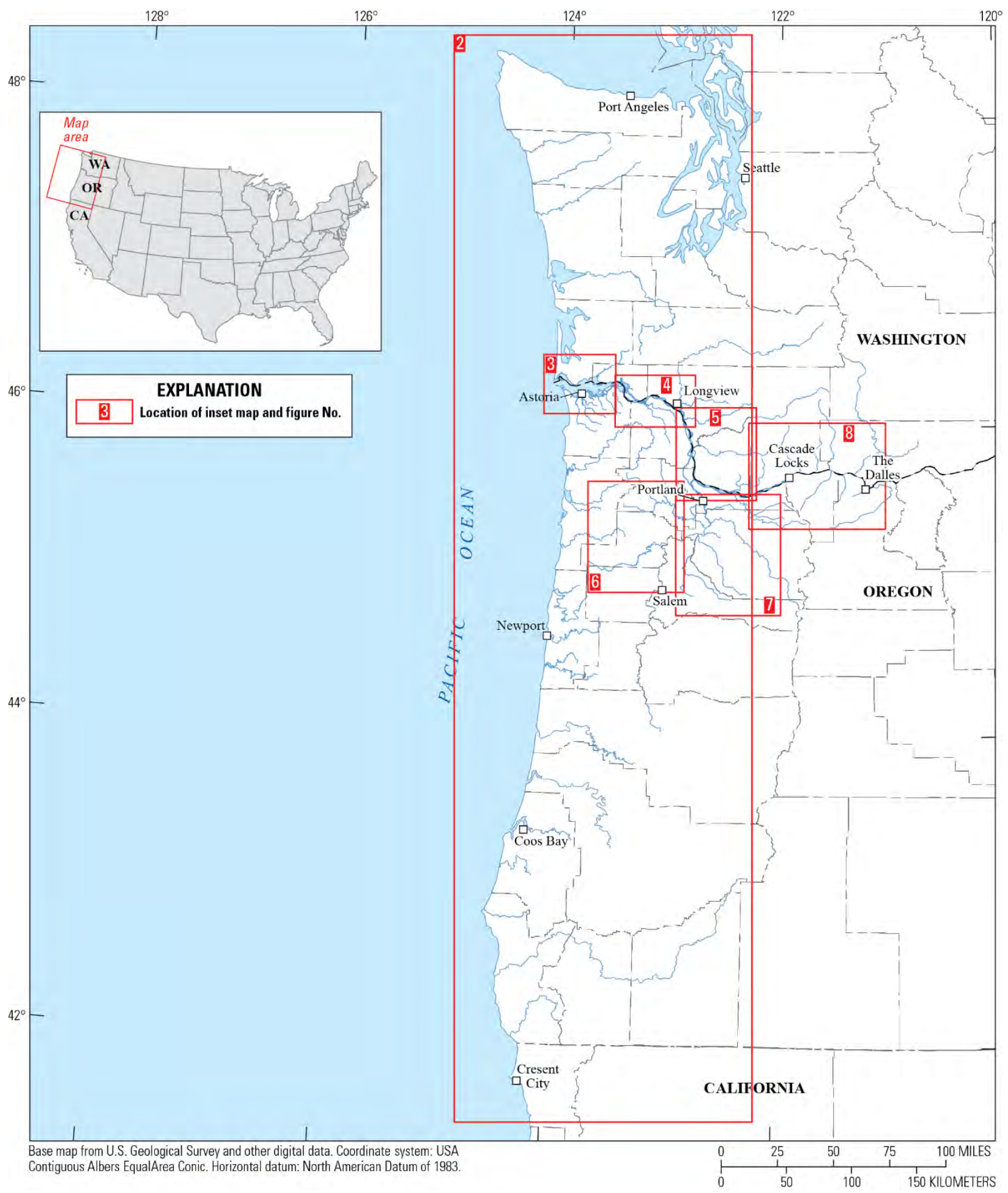

Figure 1. The Lower Columbia River flood profile study area with sub-regions. [2, Coastal Washington and Oregon; 3, Lower Columbia River Estuary; 4, Upper Columbia River Estuary; 5, Longview to Portland; 6, Willamette River Coastal; 7, Willamette River Cascades; 8, Troutdale to The Dalles.] 


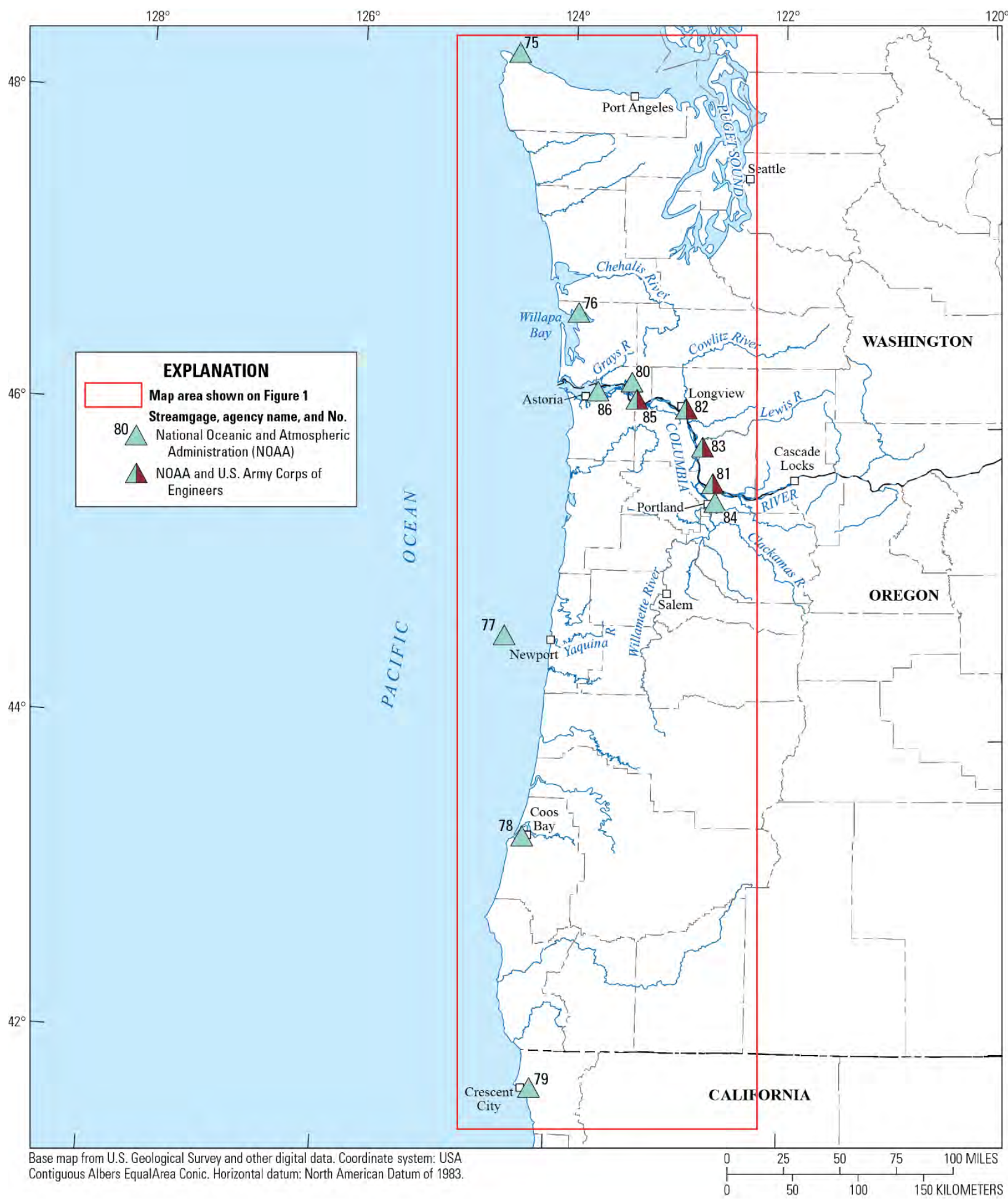

Figure 2. The coastal Washington, Oregon, and California sub-region of the Lower Columbia River flood profile study area. [The gaging stations are depicted as triangles, with the color signifying the agency/agencies that collected the data. Stations are numbered and correspond to station information provided in table 5.] 


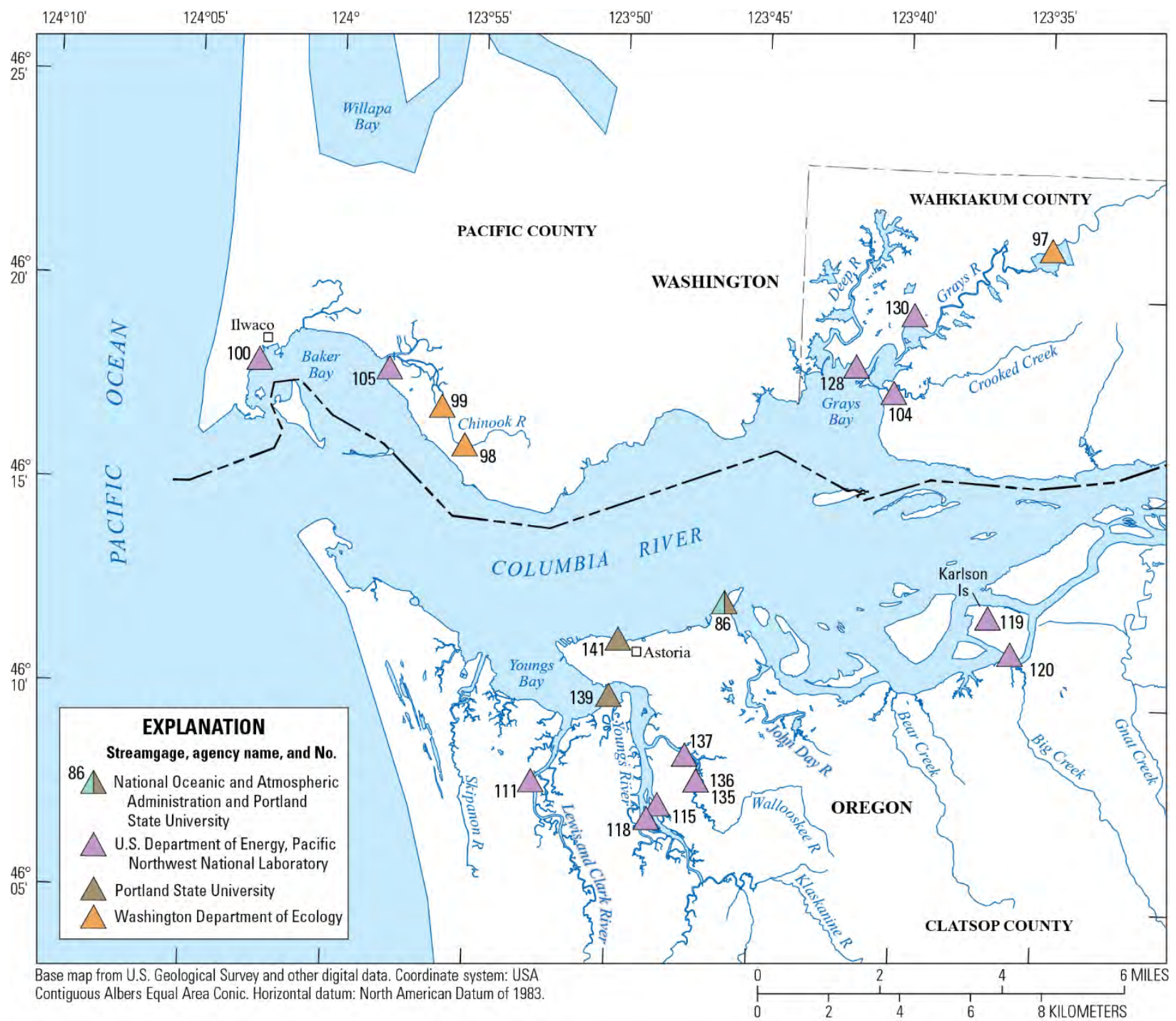

Figure 3. The Lower Columbia River Estuary sub-region of the Lower Columbia River flood profile study area, Washington and Oregon. [The gaging stations are depicted as triangles, with the color signifying the agency/agencies that collected the data. The circles depict elevation only monitoring stations. Stations are numbered and correspond to station information provided in tables $5,6,7$, and 8 .] 


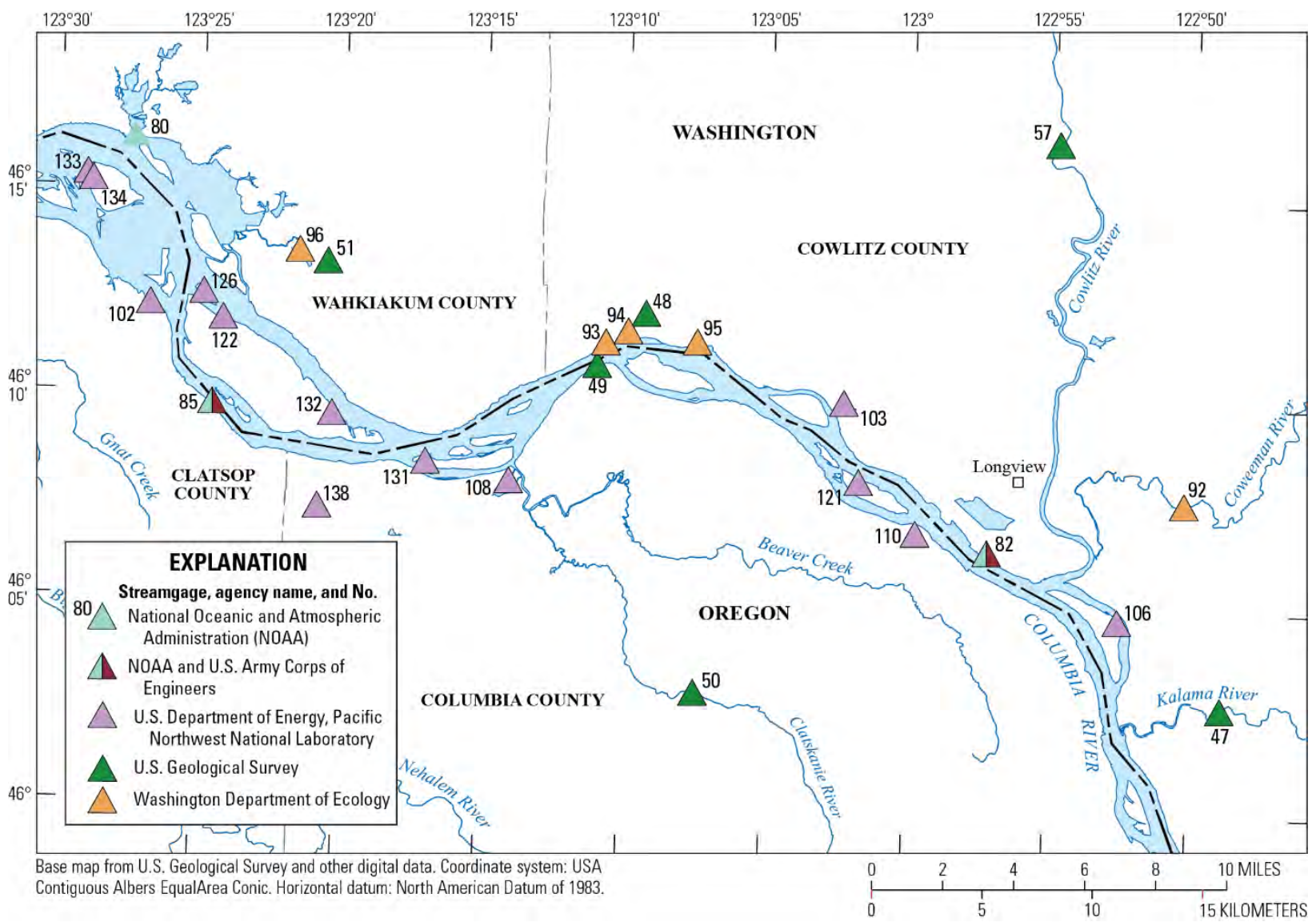

Figure 4. The Upper Columbia River Estuary sub-region of the Lower Columbia River flood profile study area, Washington and Oregon. [The gaging stations are depicted as triangles, with the color signifying the agency/agencies that collected the data. The circles depict elevation only monitoring stations. Stations are numbered and correspond to station information provided in tables 2, 5, 6, and 7.] 


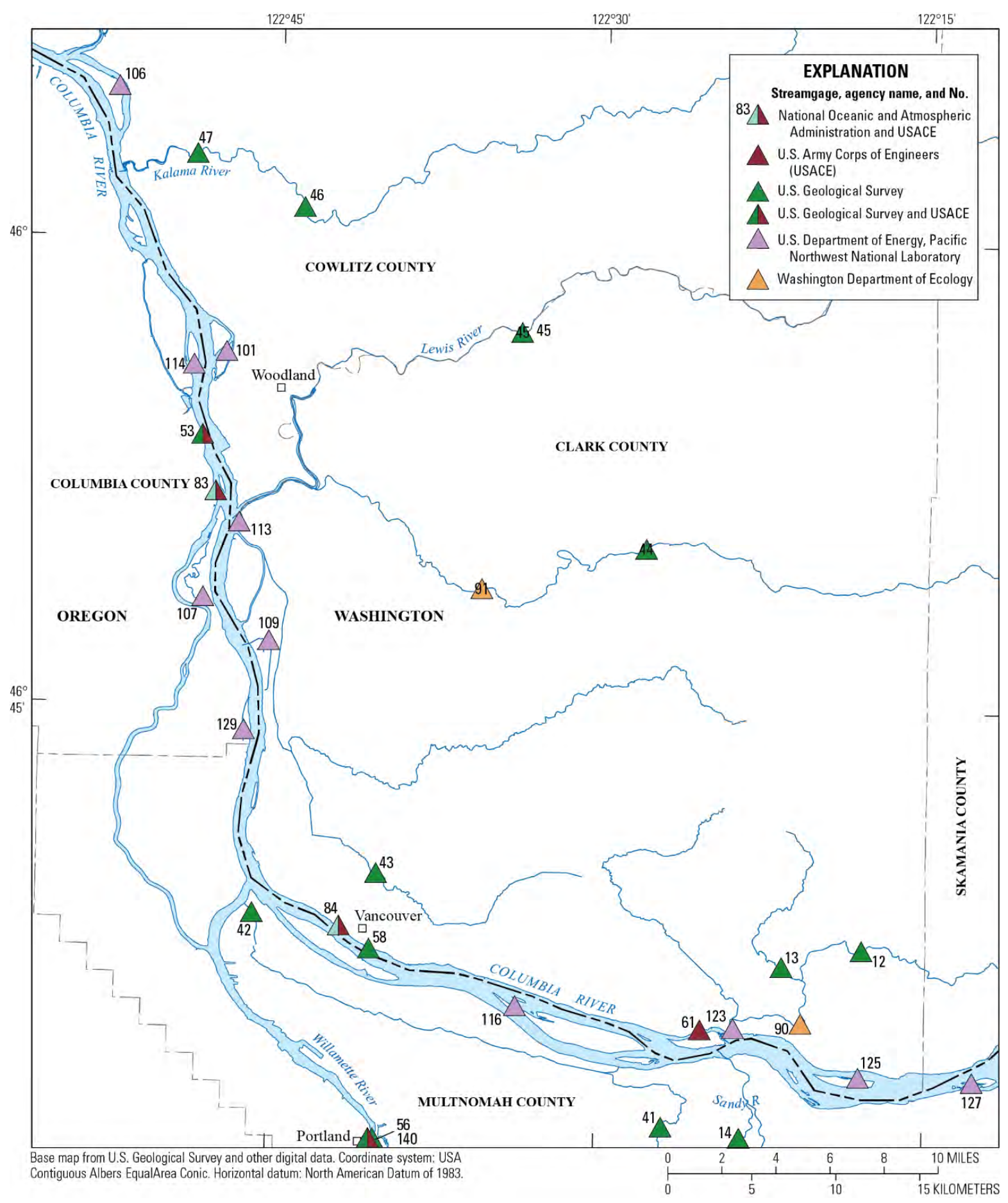

Figure 5. The Longview to Portland sub-region of the Lower Columbia River flood profile study area, Washington and Oregon. [The gaging stations are depicted as triangles, with the color signifying the agency/agencies that collected the data. The circles depict elevation only monitoring stations. Stations are numbered and correspond to station information provided in tables 2, 5, 6, 7, and 9.] 


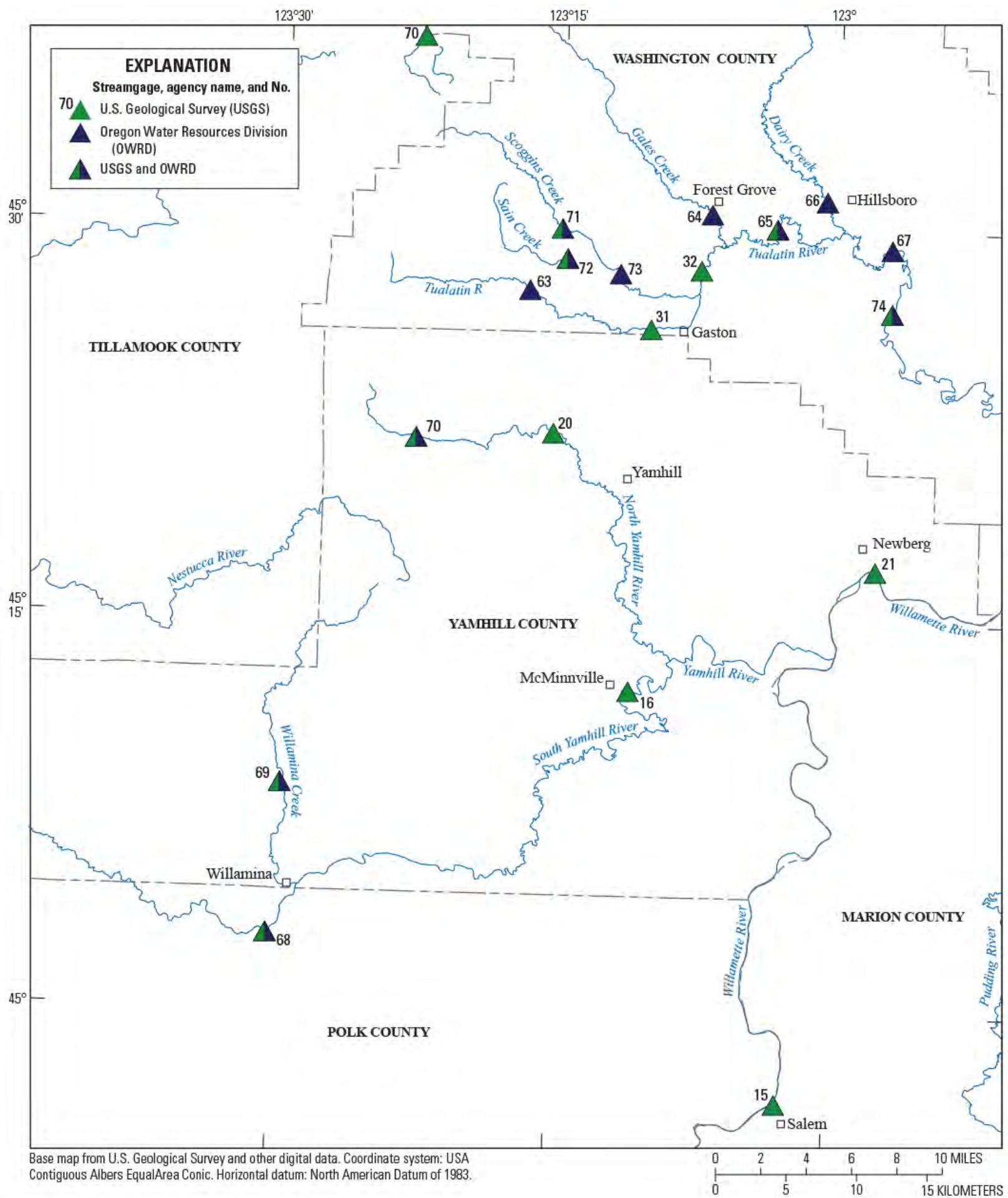

Figure 6. The Willamette River Coastal sub-region of the Lower Columbia River flood profile study area, Oregon. [The gaging stations are depicted as triangles, with the color signifying the agency/agencies that collected the data. Stations are numbered and correspond to station information provided in tables 2 and 4.] 


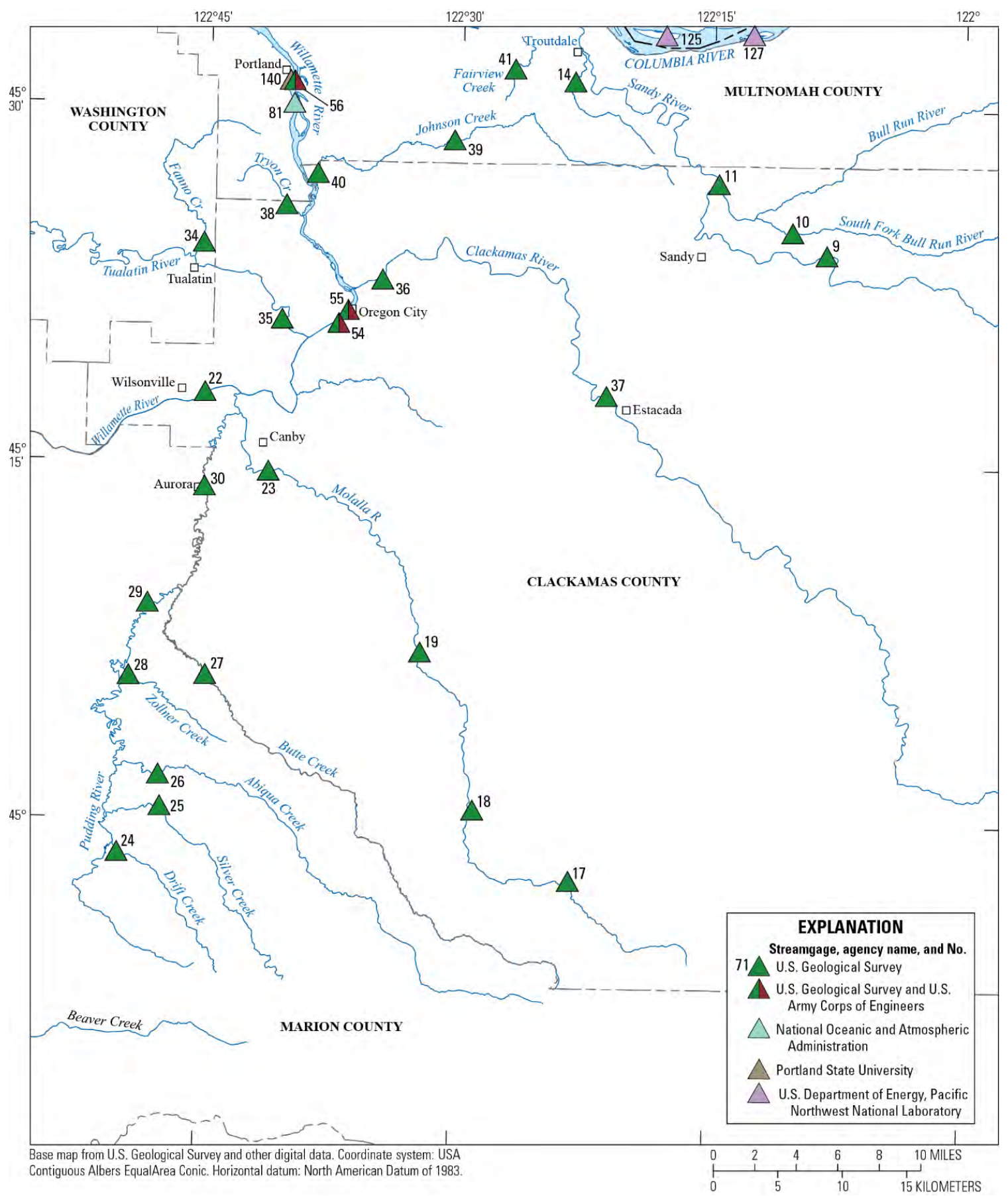

Figure 7. The Willamette River Cascades sub-region of the Lower Columbia River flood profile study area, Washington and Oregon. [The gaging stations are depicted as triangles, with the color signifying the agency/agencies that collected the data. The circles depict elevation only monitoring stations. Stations are numbered and correspond to station information provided in tables 2,5 , and 8 .] 


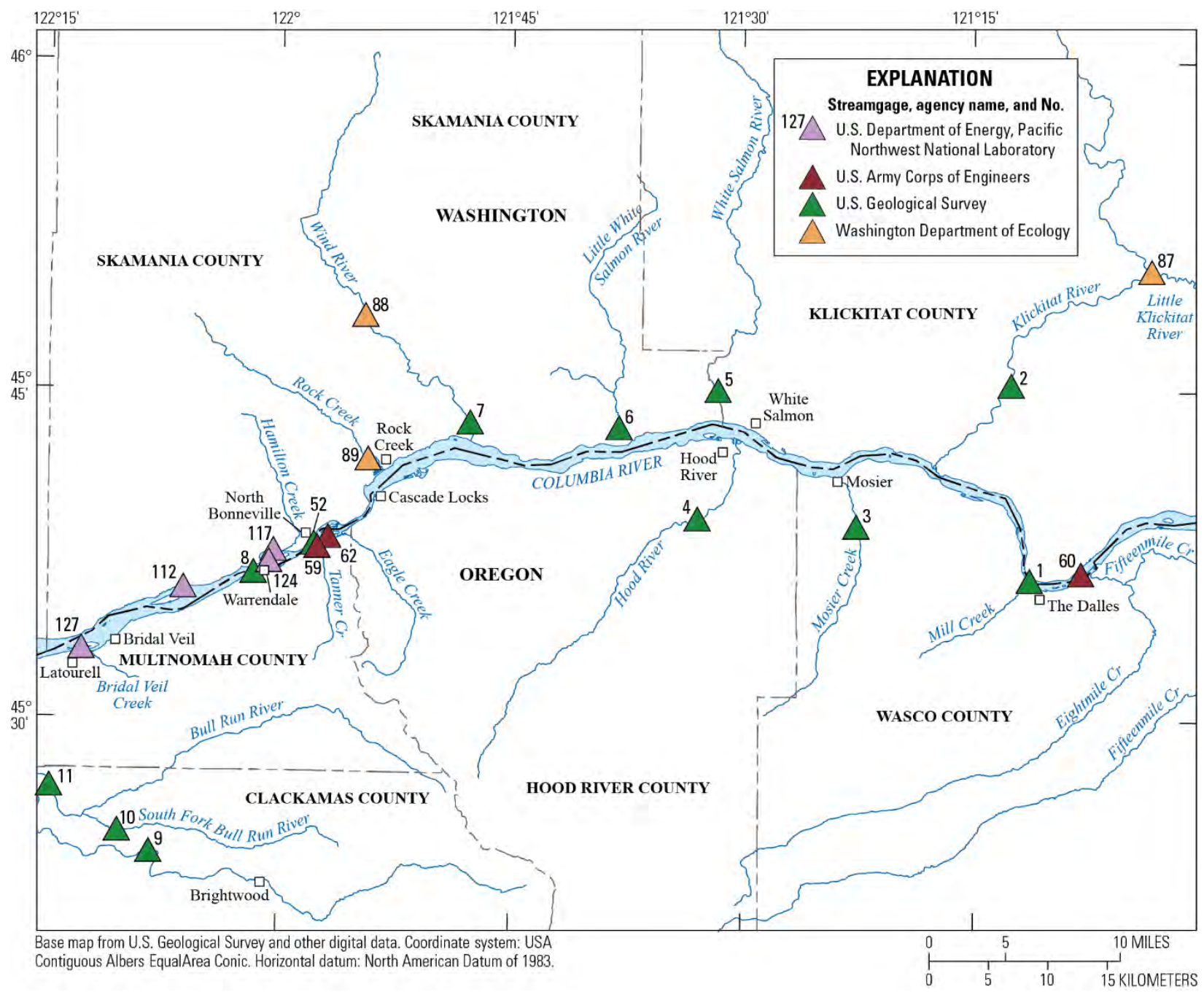

Figure 8. The Troutdale to The Dalles sub-region of the Lower Columbia River flood profile study area, Washington and Oregon. [The gaging stations are depicted as triangles, with the color signifying the agency/agencies that collected the data. The circles depict elevation only monitoring stations. Stations are numbered and correspond to station information provided in tables 2, 6, 7, and 9.] 


\title{
Appendix 1. Examination of Gaging and Feasibility of Streamflow Ratings at Willamette Falls
}

\author{
By Marc A. Stewart and Carrie L. Boudreau
}

The Willamette Falls are situated about 26 river miles upstream from the confluence of the Willamette River and the Columbia River. The normal operating range of the river above Willamette Falls, based on U.S. Geological Survey (USGS) data, is 54 feet (ft), National Geodetic Vertical Datum of 1929 (NGVD 29) in the summer to $58 \mathrm{ft}$ (NGVD 29) in the winter. The river is free flowing over Willamette Falls except for the use of flashboards and a bladder system.

Flashboards have been used to manipulate water level in the river above Willamette Falls in some form since at least 1908. The boards are typically installed annually between June and early July (fig. 1.1) and remain in place until the first high flow event in early fall. The bladders were introduced in 2008 and aid in directing the flow for fish passage. (D. Cramer, Portland General Electric, written communication, July 2, 2018). Historical water levels, above Willamette Falls, were potentially influenced by U.S. Army Corps of Engineers (USACE) navigational lock operations during summer months. That system is non-operational since December 2011 (U.S. Army Corps of Engineers, 2018).

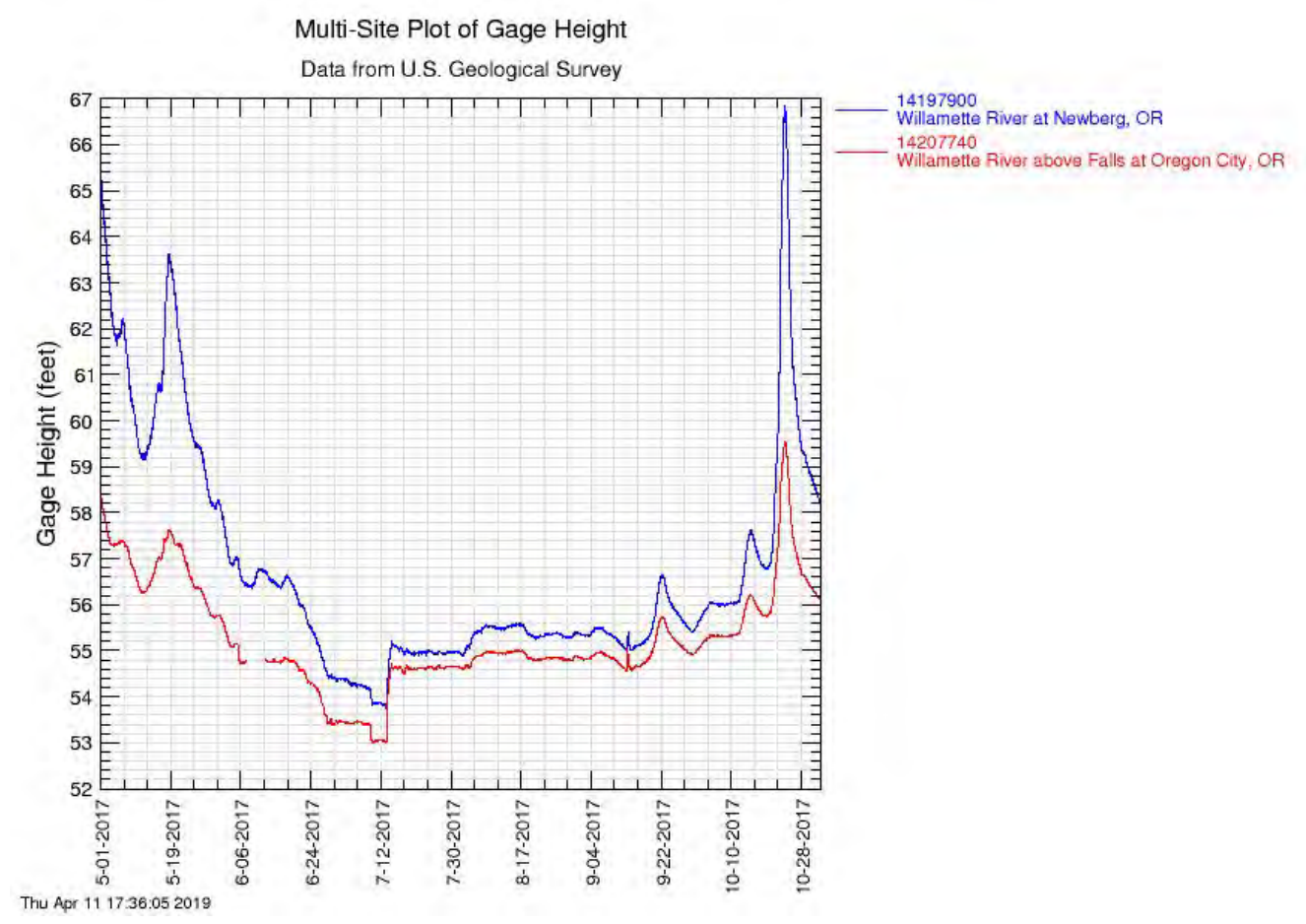


Figure 1.1. Gage height data at Willamette River above the Falls at Oregon City, Oregon, and Willamette

River at Newberg, Oregon, illustrating the effect of the flashboards for summer 2017 in which the flashboards were installed in mid-July.

\section{Summary of Key Gaging activities at Willamette Falls}

From 1909 to 1912 the USGS published daily water level data furnished by Portland Railway, Light and Power Company, a precursor to Portland General Electric (PGE). In the USGS's early Water Supply reports and archived files at the USGS office in Portland, the location was simply referred to as Willamette River at Oregon City. The gage locations were described as below Willamette Falls at a "vertical staff at the foot of the locks" or "vertical staff gage on the left bank about 1 mile above Willamette Falls." Values were published from both locations.

During 1909-12, streamflow measurements were made by the USGS on the Willamette River either below the mouth of the Clackamas River, about 1.4 miles downstream of Willamette Falls, or at the historical location of a railroad bridge 7 miles downstream of Willamette Falls. Measurements were made by boat, and inflows were subtracted as necessary, so the results represented river discharge at Willamette Falls.

The U.S. Department of Agriculture and the Weather Bureau, a precursor to the National Weather Service (NWS) also recorded water levels at this location. Their readings were made from the staff plate located below the locks (USGS files).

In 1927, the USGS, at the request of the U.S. Army Corps of Engineers made 18 streamflow measurements as well as velocity verification measurements for the turbines. More recently between 1992 and 2001, the USGS made 14 streamflow measurements above the Willamette Falls and above the Tualatin River.

The USGS has recorded water levels at both Willamette River above the Falls at Oregon City (14207740) and Willamette River Below the Falls at Oregon City (14207770) since 1976. Continuous streamflow was not computed for either of these locations. The gage above Willamette Falls is located on the right bank (from the reference frame looking downstream), 0.2 mi upstream from Willamette Falls, 1.6 mi downstream from Tualatin River, and at river mile 26.8. The gage below Willamette Falls is located on the right bank, 0.5 mi downstream from Willamette Falls, 1.4 mi upstream from Clackamas River, and at river mile 26.2

\section{Streamflow Ratings and Streamflow}

Upstream of the Willamette Falls a stage-streamflow relationship is unreliable due to backwater caused by the installation of flashboards and bladders. It appears early efforts to measure streamflow (1909-12 and 1927) were made downstream due to dangerous boating conditions above Willamette Falls. The measurements from 1992-2001 were made above the Tualatin River during non-flood conditions.

Downstream of Willamette Falls, the Willamette River is tidally influenced (fig. 1.2), preventing the development of an accurate stage-streamflow rating required to compute streamflow. Developed stage-streamflow ratings would have limited use outside of flood conditions. 


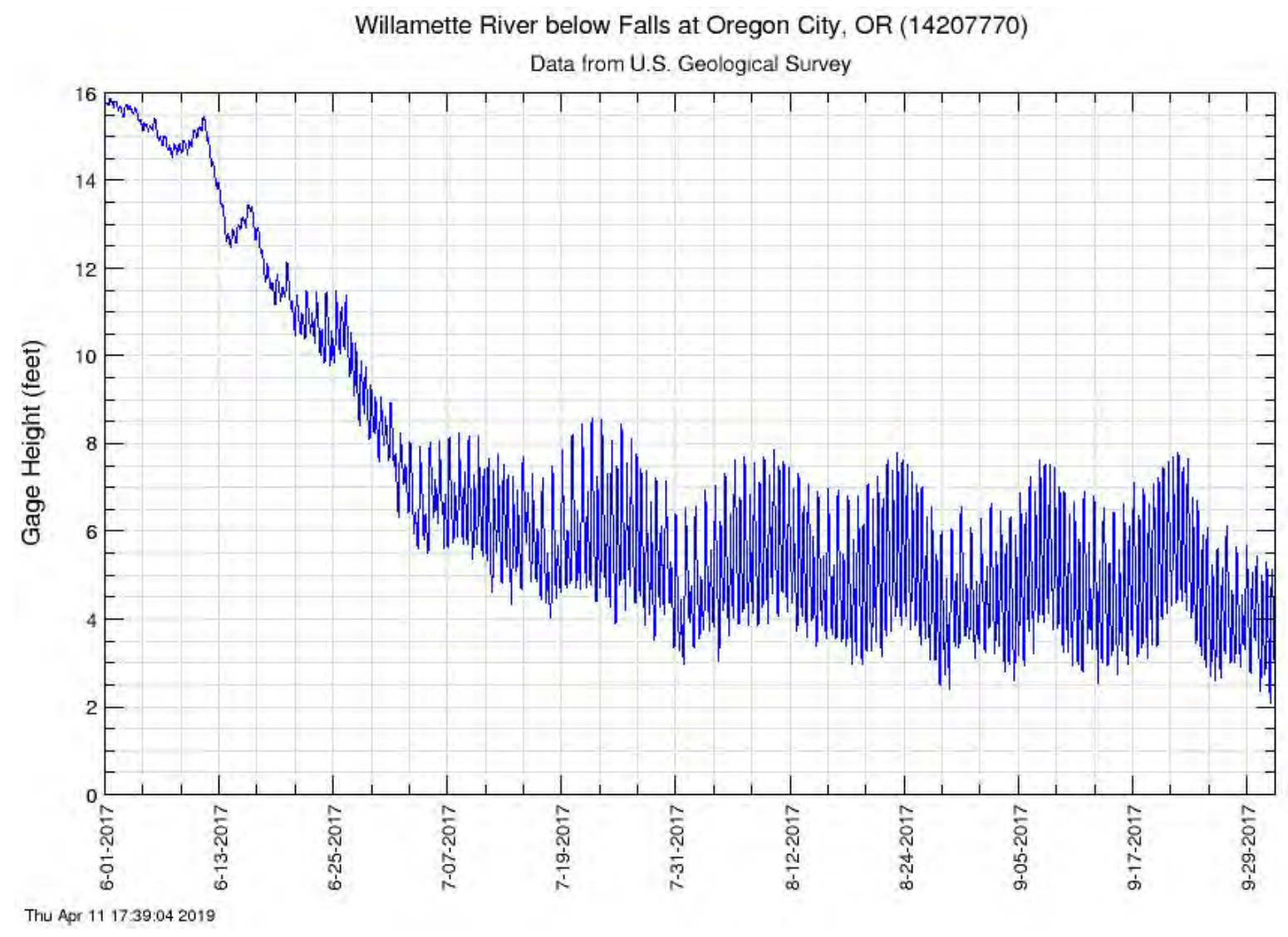

Figure 1.2. Gage height data at Willamette River below the Falls at Oregon City, Oregon; illustrating the semidiurnal tidal effect on the gage height record.

The USGS developed stage-streamflow ratings in 1912 and 1927. The ratings of data collected above Willamette Falls are only valid when flashboards are not installed due to the backwater conditions.

Of the 14 streamflow measurements made by the USGS between 1992 and 2001, 13 were correlated with available stage data from Willamette River above the Falls (14207740; fig. 1.3). 


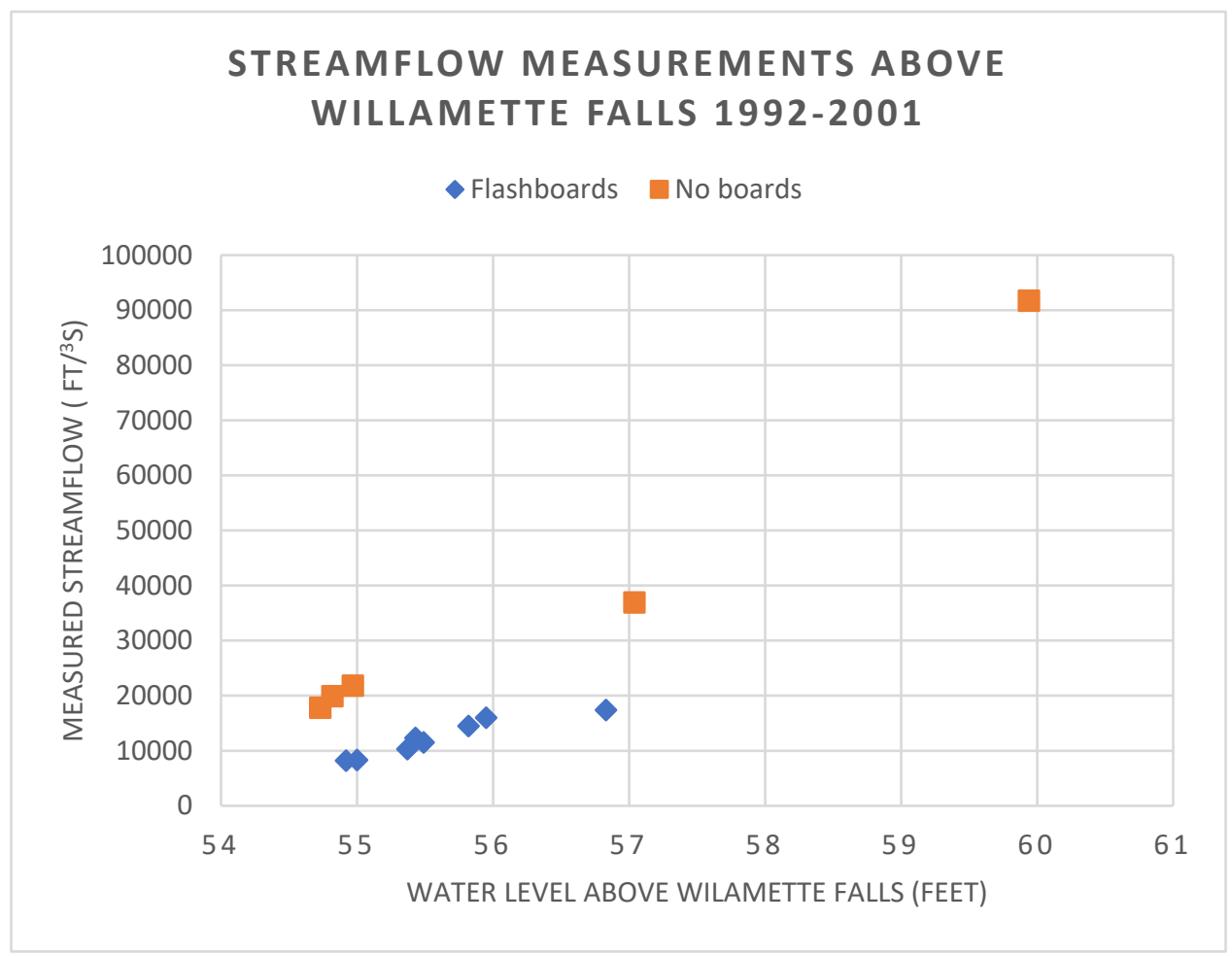

Figure 1.3. Plot of 13 streamflow measurements (cubic feet per second) and corresponding water level (feet) above the Willamette Falls.

The NWS uses a synthetic streamflow rating at Willamette Falls for internal purposes. They do not use this rating when the flashboards are in place during the summer and fall months (Stephen King, National Weather Service, written communication, Apr. 2, 2019).

The Willamette River at Newberg streamflow gage (14197900) began operation in 2001 to establish continuous streamflow data above Willamette Falls. This gage is located at river mile 50.0 and is 23.4 miles above Willamette Falls. Stage, at this location, is not affected by the flashboards or bladders.

Stage-streamflow ratings above Willamette Falls are only reliable without the flashboards or bladders. Stage-streamflow ratings below Willamette Falls are only reliable during flood conditions.

\section{Summary of Drainage Area and Inflows between Willamette River at Newberg and Willamette Falls \& Monthly Water Balance Comparisons.}

The Willamette River above the Falls streamflow gage (14207740) is at river mile 26.8 with a drainage area of 10,100 square miles. The closest streamflow gage, upstream of the Willamette Falls gage, is Willamette River at Newberg (14197900). The Newberg gage is at river mile 50.0 with a drainage area of 8,350 square miles. Major inflows between the two gages consist of the Tualatin River and the Molalla River. The Tualatin River at West Linn streamflow gage (14207500), with a drainage area of 706 square miles, accounts for 99 percent of the total drainage for the river when compared to the drainage area at the mouth of Tualatin River. On the Molalla River, two upstream gages; Molalla River near Canby (14200000) and Pudding River at 
Aurora (14202000), account for 91 percent of the drainage area when compared to the drainage area at the mouth of the Molalla River. The Willamette River at Newberg drainage area along with the drainage areas of the major inflow rivers (Tualatin and Molalla) together account for 98 percent of the total drainage area when compared to the Willamette River above Willamette Falls.

Directly downstream of Willamette Falls, the USGS measures water level at Willamette River below the Falls at Oregon City (14207700). The drainage area at the downstream and upstream water level gages are the same. The only mainstem streamflow gage downstream of Willamette Falls is the Willamette River at Portland (14211720), at river mile 12.8. The Clackamas River accounts for 98 percent of the contributing drainage area to the main river between the Willamette Falls and the Willamette River at Portland gage.

Streamflow is not computed at Willamette Falls. Currently a record of streamflow is available upstream at Willamette River at Newberg (14197900) and downstream at Willamette River at Portland (14211720). Streamflow records are also produced at significant inflows between the Willamette at Newberg and Willamette at Portland gages. These streamflow records make it feasible to determine an approximation of monthly streamflow at Willamette Falls by water balance.

Monthly water balance tables were created from October 2008 to October 2017 using available streamflow data from the gages described below (table 1.1). In 2003-07, the low flow period for 14211720 was estimated by a similar water balance method.

Table 1.1. Locations analyzed using monthly water balance tables and their associated drainage areas.

[Abbreviation: USGS, U.S. Geological Survey]

\begin{tabular}{llr}
\hline $\begin{array}{c}\text { USGS station identifier } \\
\text { or latitude, longitude } \\
\text { (decimal degrees) }\end{array}$ & \multicolumn{1}{c}{$\begin{array}{c}\text { USGS station name } \\
\text { or river location }\end{array}$} & $\begin{array}{c}\text { Drainage area } \\
\text { (square miles) }\end{array}$ \\
\hline 14207700 & Willamette River Below the Falls, at Oregon City & 10,100 \\
14207740 & Willamette River Above Falls, at Oregon City & 10,100 \\
14207500 & Tualatin River at West Linn & 706 \\
$45.3382,-122.65175$ & Near Mouth of Tualatin River & 710 \\
$45.32913,-122.65913$ & Willamette River above Tualatin River & 9,370 \\
$45.29321,-122.72089$ & Near Mouth of Molalla River & 874 \\
14200000 & Molalla River Near Canby & 323 \\
14202000 & Pudding River at Aurora & 479 \\
14197900 & Willamette River at Newberg & 8,350 \\
\hline
\end{tabular}

Two methods were used to calculate streamflow at Willamette Falls. Method 1 summed monthly streamflow based on the streamflow at Willamette River at Newberg and significant inflows between Newberg and the Willamette Falls. Method 2 summed monthly streamflow by subtracting the flow of Willamette River tributaries between the Willamette Falls and the Willamette River at Portland gage (Clackamas River at Oregon City and Johnson Creek at Milwaukie) from the streamflow values calculated for the Willamette River at Portland. Monthly data from 2008 to 2017 were averaged by month and summarized in table 1.2. When the monthly data are compared, the percent difference between the two methods are typically within 5 percent except during the summer months. 
Table 1.2. Summary of the differences between the two methods to estimate monthly streamflow at Willamette Falls, Oregon.

\begin{tabular}{rlccc}
\hline Years & Month & Method 1 & Method 2 & $\begin{array}{c}\text { Percent } \\
\text { difference }\end{array}$ \\
\hline $2008-17$ & January & 57,639 & 56,138 & -2.7 \\
$2008-17$ & February & 44,937 & 43,426 & -3.5 \\
$2008-17$ & March & 48,745 & 48,813 & 0.1 \\
$2008-17$ & April & 38,155 & 36,720 & -3.9 \\
$2008-17$ & May & 27,821 & 26,723 & -4.1 \\
$2008-17$ & June & 19,756 & 20,194 & 2.2 \\
$2008-17$ & July & 9,257 & 10,825 & 14.5 \\
$2008-17$ & August & 7,750 & 9,012 & 14 \\
$2008-17$ & September & 9,380 & 9,555 & 1.8 \\
$2008-17$ & October & 16,198 & 15,192 & -6.6 \\
$2008-17$ & November & 33,095 & 33,589 & 1.5 \\
$2008-17$ & December & 57,038 & 56,218 & -1.5 \\
\hline
\end{tabular}

Efforts to determine accurate flows at Willamette Falls during low flow periods of the year could be aided by collecting a series of streamflow measurements during the summer months near Willamette Falls.

\section{References Cited}

U.S. Army Corps of Engineers, 2018, Willamette Valley: U.S. Army Corps of Engineers, Willamette Falls Locks Web interface, accessed April 3, 2019, at https://www.nwp.usace.army.mil/willamette/locks/. 


\title{
Appendix 2. Summary of Datum for Two U.S. Geological Survey Streamflow Gages
}

\author{
By Marc A. Stewart and Carrie L. Boudreau
}

The U.S. Geological Survey (USGS) investigated the datum and history of stage data at two locations: Columbia River at Vancouver (14144700) and Willamette River at Portland (14211720). Gage-height data were collected on the north side and upstream side of the Interstate 5 (I-5) bridge for the Columbia River (fig. 2.1). The datum is Columbia River Datum (CRD), and the gage-height data can be converted to North American Vertical Datum of 1988 (NAVD 88) at this location with the addition of 5.29 feet (ft). Gage-height data at the Willamette River station were collected on the west side of the piling in the middle section of the Morrison Street Bridge (fig. 2.1). The datum of the gage is the historical USGS datum, and the water level data can be converted to NAVD 88 at this location with the addition of $4.96 \mathrm{ft}$.

\section{U.S. Geological Survey (USGS) Procedures for Datum Control}

Procedures at streamflow gages include periodic station leveling checks to ensure that gage-height measuring equipment at streamflow gages are accurately set to the established gage datum and that the relationship to the established datum is maintained over the life of the streamflow gage. Levels are run at streamflow gages, on a 1- to 3-year cycle, depending on the stability history at the location and whenever differences in gage readings are unresolved (Kenney, 2010).

The USGS term "datum of the gage" may be used to reference either a recognized datum, such as NAVD 88, National Geodetic Vertical Datum of 1929 (NGVD 29), or an arbitrary datum chosen for convenience (Kenney, 2010). Many USGS streamflow gages use an arbitrary datum plane where it is desirable for gage heights to be relatively low numbers.

\section{Columbia River at Vancouver (14144700)}

This gage is located on the I-5 Bridge. The gage datum for Columbia River at Vancouver is CRD. The CRD was established as a navigation reference plane for the Lower Columbia River (LCR) in 1912 from Astoria to Portland and extended in 1929 to the Cascade Locks on the Columbia River and up to the Willamette Falls on the Willamette River (Bondurant, 2019).

Historically, the National Weather Service (NWS) maintained and collected data at this location. Beginning in 1998, the USGS has helped with gage maintenance, data verification, and began to publish the gage-height record. Since 2002, the NWS collected data at a station approximately 1 mile downstream, referred to as Vancouver, Washington (9440083; National Oceanic and Atmospheric Administration, 2018a).

Gage-heights at USGS streamflow gages are verified by reference gage observations during site visits and the equipment is reset as necessary. The primary reference gage for this station is a staff plate mounted on the north side of the northern pier.

Gage-height data can be adjusted from station datum (CRD) to NAVD 88 with an addition of $5.29 \mathrm{ft}$. 


\section{Willamette River at Portland (14211720)}

This gage is located at the Morrison Bridge. The gage datum for the Willamette River at Portland is USGS datum. The USGS datum was determined based on historical levels run in the early 1900s, by USGS and others, to tie in the LCR and are based on the "tide station at Astoria" as the basis for Mean Sea Level (Bondurant, 2019).

USGS began collecting gage-height and velocity data at this location in 1972 to produce a continuous record of streamflow in this tidally affected reach of the river. Additional details of the 1972 velocity meter installation and flow computations are documented in a USGS OpenFile Report by Lanean and Smith (1982).

Gage-height readings are verified by reference gage observations during site visits and the equipment is reset as necessary. The primary reference gage for this station is a staff plate on the piling beneath the west control tower. The NWS also maintains a set of staff plates and a wire weight reference gage on the first pier from the left bank (PRTO3).

The vertical gage datum is $1.48 \mathrm{ft}$ above NGVD 29, and prior to 2018, the vertical gage datum was $1.55 \mathrm{ft}$ above NGVD 29. Gage-height data can be adjusted from station datum NGVD 29 to NAVD 88 with an addition of $3.48 \mathrm{ft}$ (National Oceanic and Atmospheric Administration, 2018b).

The USGS gage-heights published for Willamette River at Portland are based on the USGS datum (1.48 ft above NGVD 29), where that datum can be referenced to NAVD 88 by the addition of $4.96 \mathrm{ft}$. For example, the February 1996 peak is published as $27.74 \mathrm{ft}$ in USGS gage datum or $32.70 \mathrm{ft}$ in NAVD 1988. 


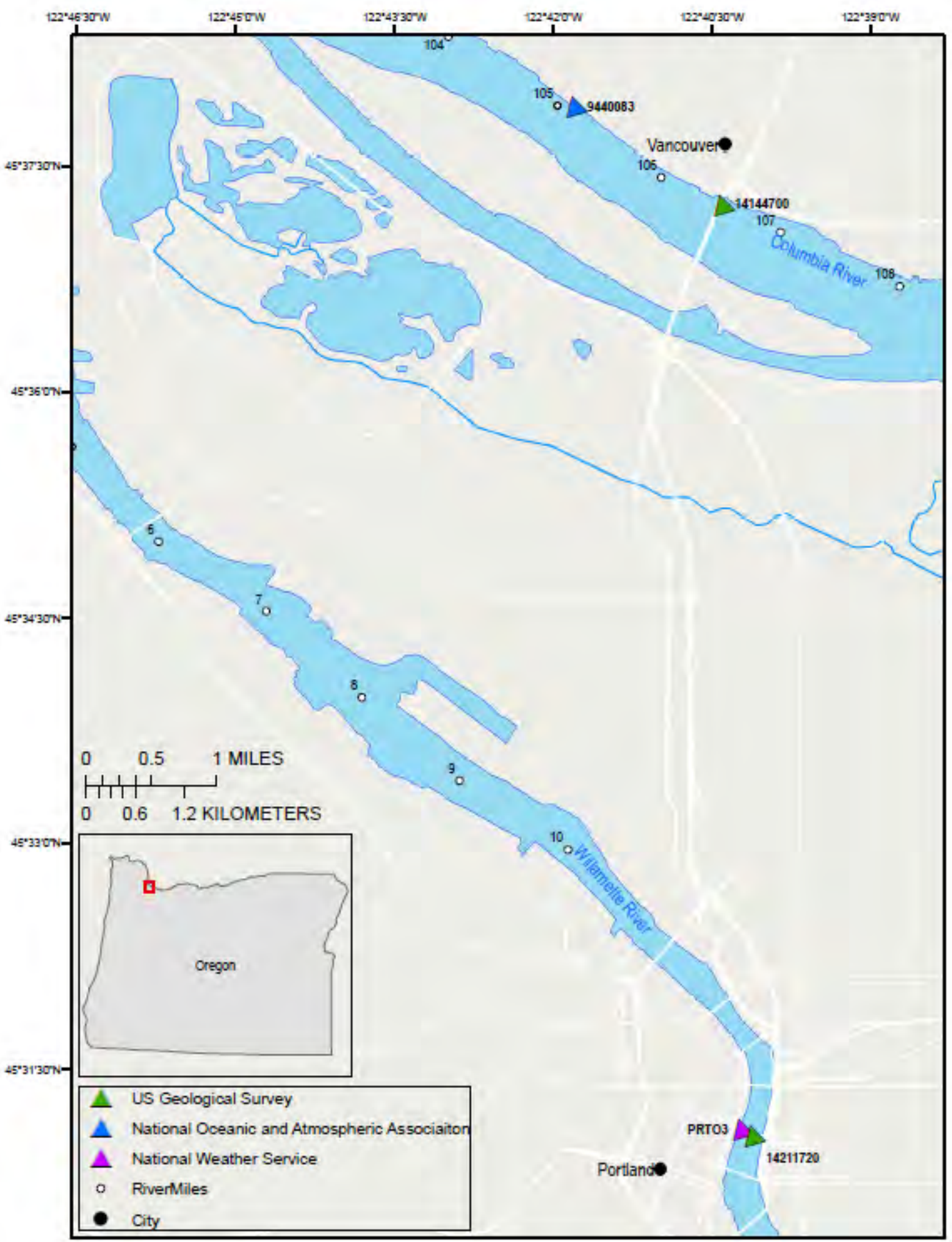

Figure 2.1. Map of the Vancouver, Washington, and Portland, Oregon, gaging station locations discussed in appendix 2. The monitoring stations are depicted as triangles, with the color signifying the agency that collected the data. 


\section{References Cited}

Bondurant, C.L., 2019, Lower Columbia and Willamette Rivers Elevation Datum Summary:

Portland, Oregon, U.S. Army Corps of Engineers.

Kenney, T.A., 2010, Levels at gaging stations: U.S. Geological Survey Techniques and Methods 3-A19, 60 p., accessed June 28, 2019, at https://pubs.er.usgs.gov/publication/tm3A19.

National Oceanic and Atmospheric Administration, 2018a, Tides and currents: National Oceanic and Atmospheric Administration web interface, accessed June 28, 2019, at https://tidesandcurrents.noaa.gov/stations.html.

National Oceanic and Atmospheric Administration, 2018b, NOAA National Geodetic SurveyPositioning America for the future: National Oceanic and Atmospheric Administration web interface, accessed June 28, 2019, at https://www.ngs.noaa.gov/PC_PROD/VERTCON/. 
Publishing support provided by the U.S. Geological Survey Science Publishing Network, Tacoma Publishing Service Center

For more information concerning the research in this report, contact the Director, Oregon Water Science Center

U.S. Geological Survey

2130 SW 5th Avenue

Portland, Oregon 97201

https://www.usgs.gov/centers/or-water 


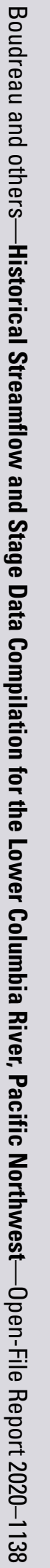

